\title{
Avian chlamydiosis in chickens: \\ from cell to population
}

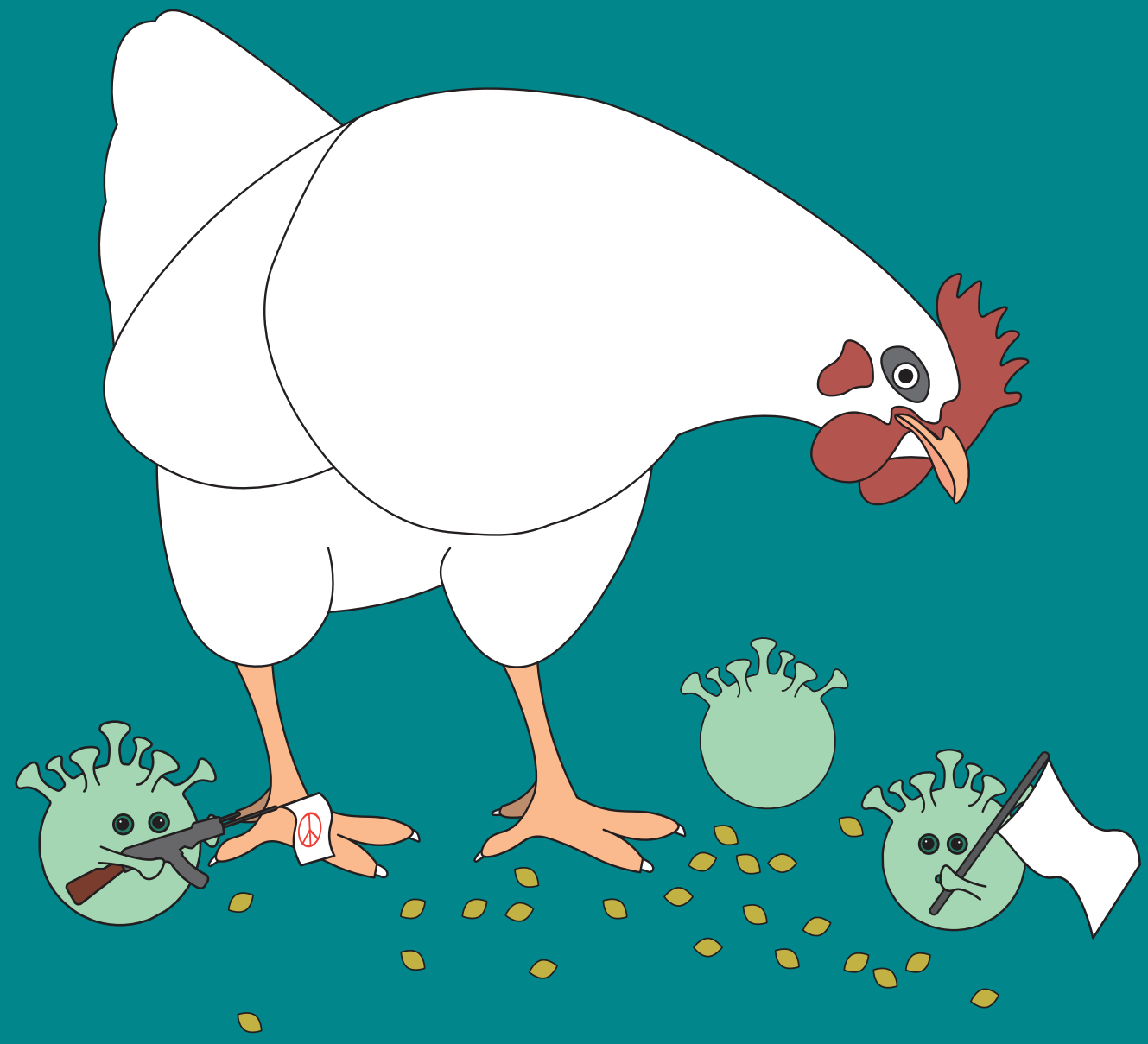

Marloes Heijne 



\section{Avian chlamydiosis in chickens: from cell to population}

Marloes Heijne 
The research in this thesis was financially supported by the Dutch Ministry of Agriculture, Nature and the Environment (WOT-01-002-005.02, WOT-01-01-002-005.13 and KB-21-006022), the Food and Consumer Product Safety Authority (NVWA) and 1 Health4Food (1 H4F) Public Private Partnership project: "Chlamydia and respiratory problems in poultry" (TKIAF-14212). Printing of this thesis was financially supported by Wageningen Bioveterinary Research and the Faculty of Veterinary Medicine of the Utrecht University.

Avian chlamydiosis in chickens: from cell to population

Marloes Heijne

PhD thesis. Utrecht University, Utrecht, the Netherlands (2021)

Design of cover: Fenna Schaap

Printing: Proefschriftmaken.nl

ISBN: 978-94-6423-527-2

DOI: https://doi.org/10.33540/843 


\section{Avian chlamydiosis in chickens: from cell to population}

\section{Aviaire chlamydiose in kippen: van cel naar populatie}

(met een samenvatting in het Nederlands)

\section{Proefschrift}

ter verkrijging van de graad van doctor aan de

Universiteit Utrecht

op gezag van de

rector magnificus, prof.dr. H.R.B.M. Kummeling, ingevolge het besluit van het college voor promoties in het openbaar te verdedigen op woensdag 8 december 2021 des middags te 4.15 uur

door

Marloes Heijne

geboren op 17 juli 1982

te Zaanstad 


\section{Promotor:}

Prof. dr. M. Nielen

\section{Copromotoren:}

Dr. A.P. Koets

Dr. J.A. van der Goot 


\section{Contents}

Chapter 1: General Introduction 9

Background on Chlamydia research and avian chlamydiosis $\quad 10$

Chlamydial bacteriology $\quad 11$

Chlamydial infections in poultry 13

$\begin{array}{ll}\text { One Health perspective } & 15\end{array}$

Scope and outline of this thesis $\quad 15$

$\begin{array}{ll}\text { References } & 17\end{array}$

Chapter 2: A cross sectional study on Dutch layer farms to investigate the prevalence and potential risk factors for different Chlamydia species 23

Abstract $\quad 24$

Introduction $\quad 25$

Materials and methods $\quad 26$

$\begin{array}{ll}\text { Sampling strategy } & 26\end{array}$

Ethics statement $\quad 27$

Laboratory tests $\quad 27$

GIS map $\quad 28$

$\begin{array}{ll}\text { Statistical analyses } & 28\end{array}$

Results $\quad 28$

Discussion 33

Funding $\quad 35$

Acknowledgements $\quad 36$

Supporting information $\quad 36$

$\begin{array}{ll}\text { References } & 37\end{array}$

Chapter 3: Genetic and phenotypic analysis of the pathogenic potential of two novel Chlamydia gallinacea strains compared to Chlamydia psittaci 41

$\begin{array}{ll}\text { Abstract } & 42\end{array}$

$\begin{array}{ll}\text { Introduction } & 43\end{array}$

$\begin{array}{ll}\text { Methods } & 44\end{array}$

Ethical statement and biosafety $\quad 44$

Sample collection, inoculum preparation and isolation of Chlamydia $\quad 44$

Titration experiments in embryonated SPF chicken eggs $\quad 47$

Histology and immunohistochemistry $\quad 47$

DNA extraction, PCR and genome sequencing $\quad 48$

Molecular typing and phylogenetic analysis $\quad 48$

$\begin{array}{ll}\text { Comparative genome analyses } & 49\end{array}$

Results $\quad 50$ 
Isolation and pathology of C. gallinacea NL_G47 and NL_F725 in

embryonated chicken eggs $\quad 50$

Assessment of virulence of C. gallinacea in embryonated eggs 52

General characteristics of the genome sequences of Dutch C. gallinacea isolates 53

Comparative genome analysis of C. gallinacea and C. psittaci 55

Discussion $\quad 59$

$\begin{array}{ll}\text { Funding } & 61\end{array}$

$\begin{array}{ll}\text { Acknowledgements } & 61\end{array}$

Supporting information $\quad 62$

References $\quad 66$

Chapter 4: Pathogenicity of Chlamydia gallinacea in chickens after oral inoculation 71

$\begin{array}{ll}\text { Abstract } & 72\end{array}$

$\begin{array}{ll}\text { Introduction } & 73\end{array}$

$\begin{array}{ll}\text { Materials and methods } & 74\end{array}$

$\begin{array}{ll}\text { Ethical statement } & 74\end{array}$

$\begin{array}{ll}\text { Inoculum } & 74\end{array}$

$\begin{array}{ll}\text { Animals and housing } & 74\end{array}$

$\begin{array}{ll}\text { Experimental design } & 74\end{array}$

$\begin{array}{ll}\text { Necropsy } & 75\end{array}$

$\begin{array}{ll}\text { PCR analyses } & 76\end{array}$

$\begin{array}{ll}\text { Histology and immunohistochemistry } & 76\end{array}$

$\begin{array}{ll}\text { Serology } & 76\end{array}$

$\begin{array}{ll}\text { Results } & 77\end{array}$

$\begin{array}{ll}\text { Clinical signs and shedding } & 77\end{array}$

Dissemination in the gastro-intestinal tract $\quad 79$

$\begin{array}{ll}\text { Dissemination to other organs } & 81\end{array}$

$\begin{array}{ll}\text { Serologic response } & 81\end{array}$

Discussion $\quad 82$

Funding $\quad 84$

Acknowledgements $\quad 84$

Supporting information $\quad 85$

$\begin{array}{ll}\text { References } & 87\end{array}$

Chapter 5: Host-pathogen interactions during experimental cross infection of Chlamydia gallinacea and Chlamydia psittaci in chickens 91

Abstract $\quad 92$

Introduction $\quad 93$

Materials and methods $\quad 94$

$\begin{array}{ll}\text { Ethical statement } & 94\end{array}$ 
$\begin{array}{ll}\text { Inocula } & 94\end{array}$

Animals and housing $\quad 94$

Experimental design $\quad 95$

Necropsy $\quad 96$

PCR analyses $\quad 97$

Histology and immunohistochemistry $\quad 97$

$\begin{array}{ll}\text { Serology } & 97\end{array}$

$\begin{array}{lr}\text { Statistics } & 98\end{array}$

Results $\quad 98$

Part 1: primary inoculation with C. gallinacea NL_G47 98

Part 2: secondary inoculations 99

Discussion 104

$\begin{array}{ll}\text { Funding } & 106\end{array}$

$\begin{array}{ll}\text { Acknowledgements } & 106\end{array}$

$\begin{array}{ll}\text { Supporting information } & 107\end{array}$

$\begin{array}{ll}\text { References } & 109\end{array}$

Chapter 6: General discussion $\quad 113$

Chlamydia infections in poultry other than layers $\quad 115$

C. gallinacea, a pathogen at all? 116

A one health perspective on C. psittaci infections in chickens $\quad 119$

Final thoughts and future research $\quad 120$

$\begin{array}{ll}\text { Take home messages } & 122\end{array}$

$\begin{array}{ll}\text { References } & 123\end{array}$

$\begin{array}{ll}\text { Summary } & 126\end{array}$

$\begin{array}{ll}\text { Samenvatting } & 128\end{array}$

$\begin{array}{ll}\text { Dankwoord } & 130\end{array}$

$\begin{array}{ll}\text { About the author } & 133\end{array}$

List of publications $\quad 134$ 
CHAPTER 1 
General Introduction 


\section{Background on Chlamydia research and avian chlamydiosis}

Avian chlamydiosis refers to disease in birds caused by bacteria from the genus Chlamydia. The term avian chlamydiosis was initially introduced to replace the terms psittacosis (disease in psittacines and humans) and ornithosis (disease in pigeons and poultry) as these are specifically caused by the bacterium Chlamydia psittaci (1). C. psittaci is zoonotic and the term psittacosis is still used to describe the disease in humans.

First detailed descriptions about psittacosis date back to 1879, when Jacob Ritter identified newly imported birds as the source of human pneumonia cases (2). In 1895, Morange introduced the term psittacosis, which refers to the Greek word for parrot, when flu-like symptoms in humans were associated with parrots (3). In the winter of 1929-1930 a psittacosis outbreak, caused by the import of parrots, resulted in a total of 766 human cases and 112 case fatalities worldwide (4). Although the number of cases were relatively small compared to other pandemics such as the current COVID-19 pandemic, the "great parrot fever" led to press headlines and further research into the cause of this mysterious disease (5).

In 1930, the morphology and life cycle of the agent causing psittacosis was described (6). At that time, the agent was classified as a virus due to its filterable size and failure to cultivate it as a bacterium (7). A few years later, C. psittaci could be cultivated on the chorioallantoic membrane (8) and in the yolk sac of embryonated chicken eggs (9). Attempts to grow Chlamydia in cell culture also started in the 1930s, but it took until 1969 before cell culture became more successful than yolk sac culture (10). In 1965, Chlamydia was finally classified as a bacterium based on studies on its morphology with electron microscopy (11)

From 1930 to 1938,174 cases of human psittacosis were reported from the Faroe Islands (12). The disease was transmitted via juvenile fulmars (Fulmarus glacialis) that were captured and subsequently prepared for cooking. This outbreak highlighted psittacosis could be transmitted by other bird species than psittacines, although parrots might have been the source of infection for the fulmars (13).

Later, Meyer showed that psittacosis had a wide host range in domestic and free-living birds; he listed 70 bird species where Chlamydia was detected (14). In 2003, this list had been extended to 469 domestic and free-living bird species comprising 30 avian orders (15). Since 2009, the taxonomy of Chlamydia has become more complex due to the discovery of new avian chlamydial species, such as Chlamydia avium, Chlamydia buteonis and Chlamydia gallinacea (16-18). Therefore, the term avian chlamydiosis is currently applied to all chlamydial infections in avian species (1). 


\section{Chlamydial bacteriology}

Chlamydia are obligate intracellular Gram-negative bacteria belonging to the family of Chlamydiaceae and order of Chlamydiales. In 2015, the two members of the family, Chlamydophila and Chlamydia, were reunited in one genus: Chlamydia (19). The genus Chlamydia currently consists of 14 species and an extending number of Candidatus species (20). The recognized and candidate species with their main host are shown in figure 1. Zoonotic potential is described for C. abortus, C. caviae, C. felis and C. psittaci $(21,22)$, while C. pneumonia and C. trachomatis are mainly known as human pathogens (23). C. avium, C. buteonis, C. gallinacea, C. psittaci and Ca. C. ibidis are predominantly found in birds (24).

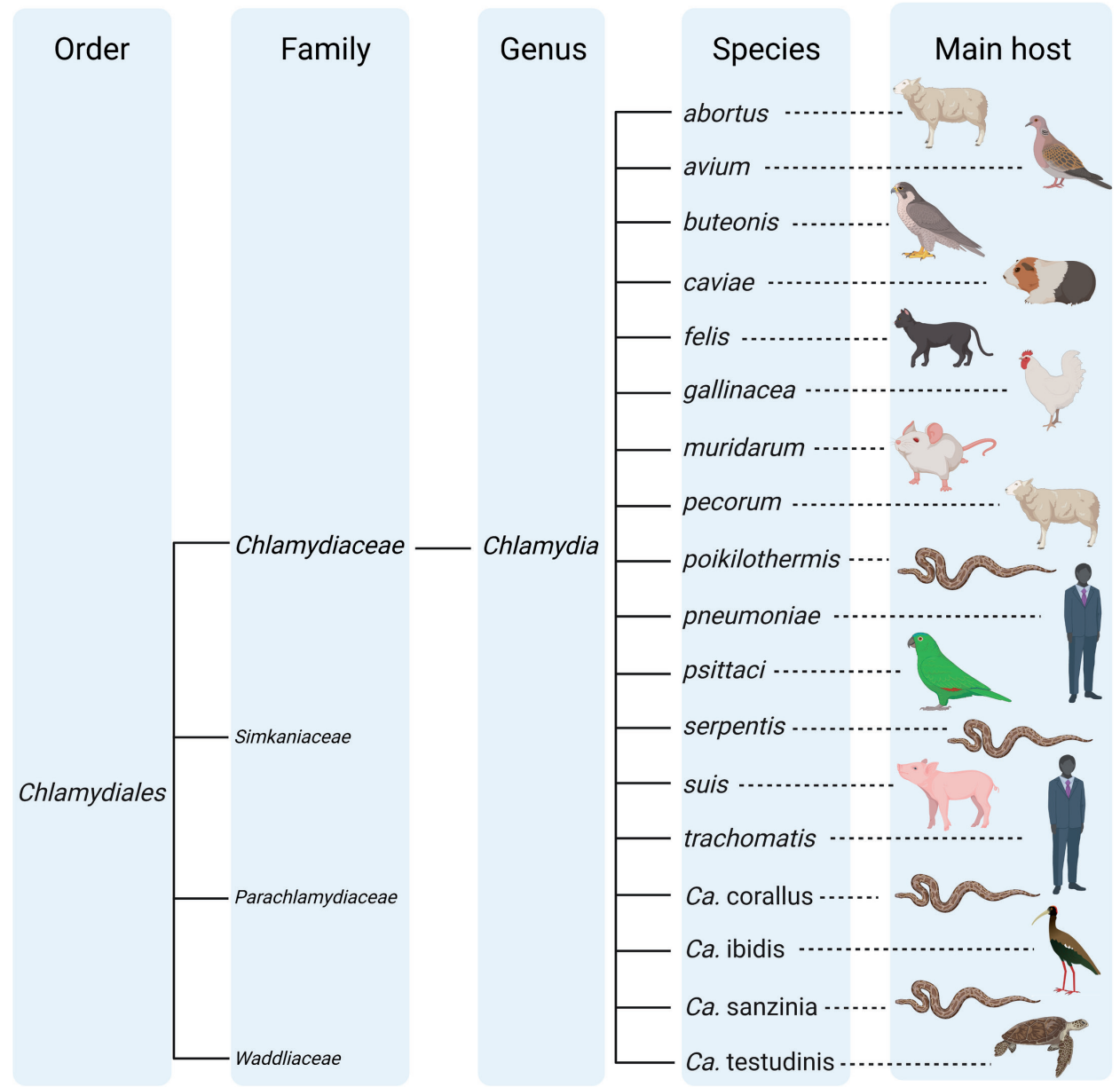

Fig. 1 Chlamydia taxonomy and host range

The current taxonomy of Chlamydia including candidate species is shown. Species are listed in alphabetical order instead of their phylogenetic relationship. The figure was created with BioRender.com. 
All Chlamydia have a biphasic life cycle with elementary bodies (EBs) and reticulate bodies (RBs), where the RBs are always located intracellular in contrast to EBs (Fig. 2). EBs are nonreplicative and infectious with a spore-like cell wall, which allows them to survive outside host cells under harsh conditions. They are relatively small particles that are electrondense due to condensed chromatin, with a diameter ranging from 0.2-0.4 $\mu \mathrm{m}$ (25). After host cell entry, EBs transform into RBs, which is the replicative and non-infectious stage. RBs are less electron-dense and about $0.5-1.5 \mu \mathrm{m}$ in size. The entire life cycle lasts about 48-72 hours depending on the chlamydial species (26).

In host cells, Chlamydia create an intracellular niche (an inclusion) from which they acquire nutrients for their survival and evade the host immune response. Their exact strategy differs between chlamydial species and hosts (27). Replication mainly takes place in epithelial cells, but some species, for example $C$. psittaci, are also able to replicate inside macrophages $(28,29)$.
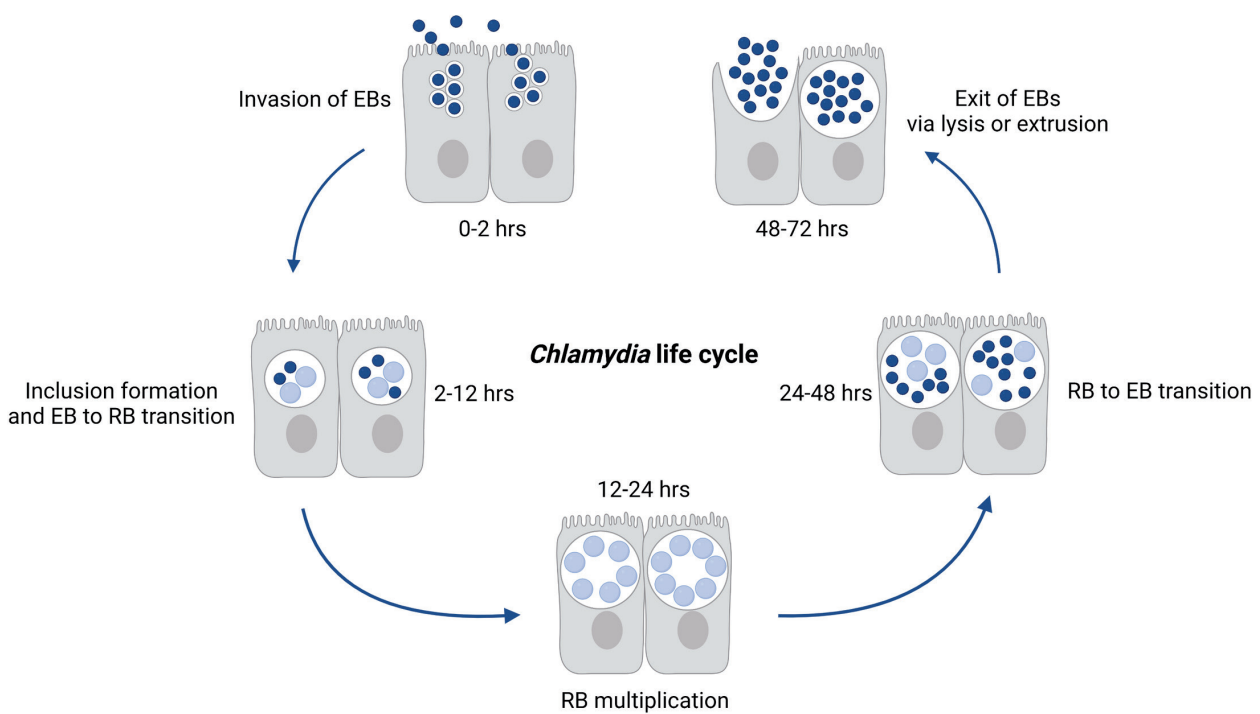

\section{Fig. 2 Chlamydia life cycle}

Elementary bodies (EBs) attach and invade host epithelial cells and form inclusions (within two hours after internalization). Between 2 and 12 hours EBs differentiate into reticulate bodies (RBs). After 12 hours RBs start to multiply by binary fission with a peak between 18 and 24 hours. Around 24 hours $R B s$ differentiate back to EBs until their release via lysis of the host cells or extrusion between 48 to 72 hours (26). The duration of the life cycle depends on the chlamydial species. The figure was created with BioRender.com.

About $60 \%$ percent of the mass of the outer membrane of Chlamydia consists of the major outer membrane protein (MOMP), which is one of the major surface antigens (30). It is frequently used as target in serology, but also for subtyping within different chlamydial 
species. In EBs MOMP probably functions as an adhesin, while in RBs it acts as a porin (27). Other important outer membrane constituents are the chlamydial lipopolysaccharide (LPS), OmcA and OmcB, polymorphic membrane proteins (pmps) and the Type III secretion system (T3SS). The T3SS forms a needle like structure allowing the export of several effector proteins into the host cell, and is considered one of the most important virulence factors (31).

Chlamydia have relatively small genomes compared to many other bacteria, with an average genome size of 1 mega base pairs (Mbp) carrying about 880 to 1050 coding sequences (CDS). Recent genomic comparison between 12 different chlamydial species and 33 strains revealed that all species share about $80 \%$ of their genome involving 784 genes (32). Outside this core genome, the most variable regions are genes encoding pmps, genes encoding T3SS effector proteins and the plasticity zone (33). The plasticity zone is a region near the replication terminus with genes that encode for a cytotoxin and membrane attack complex/perforin protein (MACPF) $(23,33)$. All these variable regions are related to virulence, host interaction and/or host tropism $(23,33)$. In addition, most strains carry plasmids that might contribute to their virulence (23).

\section{Chlamydial infections in poultry}

Until 2009, C. psittaci was considered the predominant chlamydial species in poultry. $C$. psittaci is most closely related to C. abortus, C. caviae and C. felis and can be subdivided in at least nine different genotypes (former serotypes) based on the gene sequence coding for MOMP, ompA. The genotypes are associated with different host species. Avian strains (genotype $\mathrm{A}$ to $\mathrm{F}$ ) are considered to be more virulent than mammalian strains (genotype M56 and WC). In poultry, genotype $C$ and $E / B$ are mostly found in ducks and genotype $D$ and E/B in turkeys (11). No specific genotype is associated with infections in chickens. Other more discriminatory typing schemes, such as Multi Locus Sequence typing (MLST), which is based on sequence analysis of seven housekeeping genes, identified at least 12 different $C$. psittaci sequence types (ST) that could also be associated with different host species (34).

C. psittaci is widespread, but its prevalence varies between genotypes and bird species (35). Chickens were thought to be relatively resistant to C. psittaci infection, but a Belgian study in 2014 reported 6/7 broiler breeder, 7/7 broiler and 5/5 layer farms PCR and culture positive for C. psittaci in pharyngeal swabs (36). Other studies in Belgium and NorthernFrance from 2010 and 2013 also reported a high prevalence of C. psittaci in chickens determined with PCR, culture (on pharyngeal swabs and tissues) and/or serology $(37,38)$. 
In birds, C. psittaci is excreted in faecal droppings and nasal discharges (35). Faecal shedding is intermittent and can be activated through stress factors such as transport and overcrowding (35). Shedding can last for several months (35). The main route of transmission is the respiratory route via inhalation of dried faecal material or respiratory exudate (11). Transmission can also occur via ingestion. Vertical transmission and mechanical transmission via biting flies, lice or mites have been described, but are not considered important transmission routes (11).

In general, clinical signs and pathogenesis of C. psittaci depend on the strain, host, host age and (environmental) stressors (11). In turkeys, severe systemic infections and mortality have been described $(39,40)$. Ducks seem to be less susceptible to C. psittaci infection and are mainly subclinically infected $(41,42)$. Experimental infections with $C$. psittaci in chickens can remain asymptomatic, but can also result in severe systemic illness and mortality $(38,43,44)$.

In 2009, a new chlamydial species was detected in poultry, later classified as Chlamydia gallinacea $(16,17)$. C. gallinacea is widespread, with reports from Asia (45), Australia (46), Europe (47-51), North-(52) and South-America(53). Most studies have been conducted in chickens in which C. gallinacea is highly prevalent. Since its discovery, C. gallinacea has also been found in other species such as pigeons, woodcocks, a parrot, an ultramarine grosbeak and cattle $(1,45,46,54-56)$.

Genome analysis showed that $C$. gallinacea is a separate species, based on an average nucleotide identity of less than 81 percent with C. avium as the most closely related species (17). Strain $08-1274 / 3^{\top}$ is considered the type strain and was isolated from a chicken in France (17). Molecular studies using outer membrane protein A (ompA) genotyping or MLST showed C. gallinacea is diverse, with at least 13 different ompA types and 15 different sequence types (ST) in 25 strains and without any host or geographical association (45, 57). Whether this diversity contributes to differences in pathogenicity of $C$. gallinacea strains is currently unknown.

C. gallinacea is shed in faecal droppings in high quantities, thus contamination of the environment might play an important role in transmission. The main route of transmission is considered to be the faecal-oral route (56). In contrast to C. psittaci, airborne transmission via dried faecal material does not seem to occur (58). Vertical transmission has been proposed after detection of C. gallinacea in the albumen and yolk of embryonated eggs. However, environmental contamination via penetration through the egg shell could not be excluded (58). Cases of mechanical transmission via biting flies, lice or mites have not been described. 
Infections with C. gallinacea in chickens appear to be mainly asymptomatic, but reduced weight gain in broilers has been observed $(45,47,48)$. Experimental data on the pathogenesis of C. gallinacea in poultry are limited thus far, and show C. gallinacea is mainly residing in the gut, although it has been detected with PCR in blood, lung, heart, liver, trachea, kidney, pancreas and spleen samples $(16,45,58)$. Additional research into the pathogenicity is required to assess the importance of $C$. gallinacea as a poultry pathogen.

\section{One Health perspective}

The Dutch Q fever outbreak from to 2007 to 2010 highlighted the possible impact of environmental transmission of zoonotic pathogens from farm animals to humans. After the outbreak, studies were initiated to assess the public health risks of intensive farming in densely populated areas. One observation was a higher incidence of human pneumonia cases with unknown aetiology in the direct proximity of poultry farms (59). About the same time, studies from surrounding countries showed C. psittaci was more prevalent in chickens than previously assumed $(36,38,60)$. C. psittaci is a zoonosis and infection in humans can result in severe pneumonia (61), but around 2012 its occurrence in Dutch poultry farms was unknown. The annual number of notified human C. psittaci cases in the Netherlands varies between 25 and 85 . However, disease burden calculations estimated that more than 1500 human cases might remain undiagnosed since awareness of psittacosis is generally low (61) and C. psittaci is often not included in routine diagnostic panels (62).

In addition, C. gallinacea was described as a new widespread chlamydial species in poultry with a possible zoonotic potential, since transmission to humans was suspected in slaughterhouse workers with signs of pneumonia $(1,16)$. The lack of understanding of the prevalence of (potential) zoonotic chlamydial species in Dutch poultry triggered the research in this thesis.

\section{Scope and outline of this thesis}

In this thesis we aimed to gain insight into the prevalence of $C$. psittaci and C. gallinacea in poultry, specifically chicken layers, in the Netherlands. Moreover, we aimed to further elucidate the pathogenicity of C.gallinacea and studied possible cross protection between chlamydial species in chickens.

In Chapter $\mathbf{2}$ the prevalence of C. psittaci and C. gallinacea in Dutch layers was investigated as part of a surveillance program for zoonotic pathogens in farm animals. Furthermore, 
potential risk factors were investigated that could be associated with the presence of $C$. gallinacea, such as the occurrence of clinical signs or a higher mortality rate.

In Chapter $\mathbf{3}$ and $\mathbf{4}$ the pathogenicity of C. gallinacea was further analysed. First, two novel strains (NL_G47 and NL_F725) were isolated and compared using an in vivo infection model in embryonated chicken eggs and comparative genomics. Subsequently, the primary pathogenicity of $C$. gallinacea in chickens was investigated in three consecutive animal experiments. Chickens were inoculated orally with one of the Dutch isolates (NL_G47) and shedding was measured in throat and cloacal swabs during 11 days post infection. In addition, tissue dissemination was investigated through sequentially sacrificing of animals and blood was collected to measure a serologic response.

Chapter 5 describes the results of cross infection experiments with C. psittaci and C. gallinacea in chickens. We hypothesized that an infection with $C$. gallinacea might protect against an infection with C. psittaci. To investigate this hypothesis, chickens were inoculated with C. gallinacea NL_G47 and, after five weeks, inoculated with either a different strain of $C$. gallinacea (NL_F725) or with a strain of $C$. psittaci. These treatments were compared to single exposure with either $C$. gallinacea (NL_F725) or C. psittaci. Reduced shedding or tissue dissemination in the groups that had been pre-inoculated with $C$. gallinacea NL_G47 would be an indication of possible cross protection between C. gallinacea strains and/or C. psittaci.

The last chapter (Chapter 6) discusses whether results in layers might be extrapolated to broilers or other poultry species, whether $C$. gallinacea should be considered a pathogen and how the findings of this thesis should be interpreted from a One Health perspective. 


\section{References}

1. Sachse K, Laroucau K, Vanrompay D. Avian Chlamydiosis. Current Clinical Microbiology Reports. 2015;2(1):10-21.

2. Ritter J. Ein Beitrag zur Frage des Pneumotyphus: (Eine Hausepidemie in Uster betreffend.) Inaug.-Diss. von Zürich: Dr. v. J. B. Hirschfeld; 1879.

3. Morange A. De la psittacose ou infection spéciale déterminée par les perruches: Médecine de Paris; 1895.

4. Pospischil A. From disease to etiology: historical aspects of Chlamydia-related diseases in animals and humans. Drugs Today. 2009;45(Suppl):141-6.

5. Honigsbaum M. The Pandemic century: One hundred years of panic, hysteria, and hubris: WW Norton \& Company; 2019.

6. Bedson SP, Bland JOW. A Morphological Study of Psittacosis Virus, with the Description of a Developmental Cycle: Br J Exp Pathol. 1932 Oct;13(5):461-6.

7. Bedson SP, Western GT. Observations on the Virus of Psittacosis. Br J Exp Pathol. 1930;11(6):50211.

8. Burnet FM, Rountree PM. Psittacosis in the developing egg. The Journal of Pathology and Bacteriology. 1935;40(3):471-81.

9. Yanamura HY, Meyer KF. Studies on the Virus of Psittacosis Cultivated in vitro. The Journal of infectious diseases. 1941;68(1):1-15.

10. Gordon FB, Harper IA, Quan AL, Treharne JD, Dwyer RS, Garland JA. Detection of Chlamydia (Bedsonia) in certain infections of man. I. Laboratory procedures: comparison of yolk sac and cell culture for detection and isolation. The Journal of infectious diseases. 1969;120(4):451-62.

11. Harkinezhad T, Geens T, Vanrompay D. Chlamydophila psittaci infections in birds: a review with emphasis on zoonotic consequences. Veterinary microbiology. 2009;135(1-2):68-77.

12. Rasmussen R. Ueber eine durch Sturmvögel übertragbare Lungenerkrankung auf den Faröern. Zentralbl f Bakt. 1938;143(Dec. 30):89-93.

13. Herrmann B, Persson H, Jensen JK, Joensen HD, Klint M, Olsen B. Chlamydophila psittaci in Fulmars, the Faroe Islands. Emerging infectious diseases. 2006;12(2):330-2.

14. Meyer KF, Eddie B. Reservoirs of the Psittacosis agent. Acta Trop. 1952;9(3):204-15.

15. Kaleta EF, Taday EM. Avian host range of Chlamydophila spp. based on isolation, antigen detection and serology. Avian pathology : journal of the WVPA. 2003;32(5):435-61.

16. Laroucau K, Vorimore F, Aaziz R, Berndt A, Schubert E, Sachse K. Isolation of a new chlamydial agent from infected domestic poultry coincided with cases of atypical pneumonia among slaughterhouse workers in France. Infection, genetics and evolution : journal of molecular epidemiology and evolutionary genetics in infectious diseases. 2009;9(6):1240-7.

17. Sachse K, Laroucau K, Riege K, Wehner S, Dilcher M, Creasy HH, et al. Evidence for the existence of two new members of the family Chlamydiaceae and proposal of Chlamydia avium sp. nov. and Chlamydia gallinacea sp. nov. Systematic and applied microbiology. 2014;37(2):79-88. 
18. Laroucau K, Vorimore F, Aaziz R, Solmonson L, Hsia RC, Bavoil PM, et al. Chlamydia buteonis, a new Chlamydia species isolated from a red-shouldered hawk. Systematic and applied microbiology. 2019;42(5):125997.

19. Sachse K, Bavoil PM, Kaltenboeck B, Stephens RS, Kuo CC, Rossello-Mora R, et al. Emendation of the family Chlamydiaceae: proposal of a single genus, Chlamydia, to include all currently recognized species. Systematic and applied microbiology. 2015;38(2):99-103.

20. Laroucau K, Ortega N, Vorimore F, Aaziz R, Mitura A, Szymanska-Czerwinska M, et al. Detection of a novel Chlamydia species in captive spur-thighed tortoises (Testudo graeca) in southeastern Spain and proposal of Candidatus Chlamydia testudinis. Systematic and applied microbiology. 2020;43(2):126071.

21. Rodolakis A, Yousef Mohamad K. Zoonotic potential of Chlamydophila. Veterinary microbiology. 2010;140(3-4):382-91.

22. Ramakers BP, Heijne M, Lie N, Le TN, van Vliet M, Claessen VPJ, et al. Zoonotic Chlamydia caviae Presenting as Community-Acquired Pneumonia. The New England journal of medicine. 2017;377(10):992-4.

23. Elwell C, Mirrashidi K, Engel J. Chlamydia cell biology and pathogenesis. Nat Rev Microbiol. 2016;14(6):385-400.

24. Zareba-Marchewka K, Szymanska-Czerwinska M, Niemczuk K. Chlamydiae - What's New? J Vet Res. 2020;64(4):461-7.

25. Kuo CC, Stephens, R.S., Bavoil, P.M. and Kaltenbroeck, B. Chlamydia, Bergey's Manual of Systematics of Archae and Bacteria2015.

26. Bastidas RJ, Elwell CA, Engel JN, Valdivia RH. Chlamydial intracellular survival strategies. Cold Spring Harb Perspect Med. 2013;3(5):a010256.

27. Gitsels A, Sanders N, Vanrompay D. Chlamydial Infection From Outside to Inside. Frontiers in microbiology. 2019;10:2329.

28. Beeckman DS, Vanrompay DC. Biology and intracellular pathogenesis of high or low virulent Chlamydophila psittaci strains in chicken macrophages. Veterinary microbiology. 2010;141(34):342-53.

29. Herweg JA, Rudel T. Interaction of Chlamydiae with human macrophages. FEBS J. 2016;283(4):608-18.

30. Christensen S, McMahon RM, Martin JL, Huston WM. Life inside and out: making and breaking protein disulfide bonds in Chlamydia. Crit Rev Microbiol. 2019;45(1):33-50.

31. Radomski N, Einenkel R, Muller A, Knittler MR. Chlamydia-host cell interaction not only from a bird's eye view: some lessons from Chlamydia psittaci. FEBS Lett. 2016;590(21):3920-40.

32. Holzer M, Barf LM, Lamkiewicz K, Vorimore F, Lataretu M, Favaroni A, et al. Comparative Genome Analysis of 33 Chlamydia Strains Reveals Characteristic Features of Chlamydia psittaci and Closely Related Species. Pathogens. 2020;9(11).

33. Voigt A, Schofl G, Saluz HP. The Chlamydia psittaci genome: a comparative analysis of intracellular pathogens. PloS one. 2012;7(4):e35097. 
34. Pannekoek Y, Dickx V, Beeckman DS, Jolley KA, Keijzers WC, Vretou E, et al. Multi locus sequence typing of Chlamydia reveals an association between Chlamydia psittaci genotypes and host species. PloS one. 2010;5(12):e14179.

35. Vanrompay D. Avian Chlamydiosis. Diseases of Poultry2020. p. 1086-107.

36. Lagae S, Kalmar I, Laroucau K, Vorimore F, Vanrompay D. Emerging Chlamydia psittaci infections in chickens and examination of transmission to humans. Journal of medical microbiology. 2014;63(Pt 3):399-407.

37. Dickx V, Geens T, Deschuyffeleer T, Tyberghien L, Harkinezhad T, Beeckman DS, et al. Chlamydophila psittaci zoonotic risk assessment in a chicken and turkey slaughterhouse. Journal of clinical microbiology. 2010;48(9):3244-50.

38. Yin L, Kalmar ID, Lagae S, Vandendriessche S, Vanderhaeghen W, Butaye $P$, et al. Emerging Chlamydia psittaci infections in the chicken industry and pathology of Chlamydia psittaci genotype $B$ and $D$ strains in specific pathogen free chickens. Veterinary microbiology. 2013;162(2-4):740-9.

39. Page LA. Experimental Ornithosis in Turkeys. Avian diseases. 1959;3(1):51-66.

40. Vanrompay D, Ducatelle R, Haesebrouck F, Hendrickx W. Primary pathogenicity of an European isolate of Chlamydia psittaci from turkey poults. Veterinary microbiology. 1993;38(1-2):103-13.

41. Thierry S, Vorimore F, Rossignol C, Scharf S, Sachse K, Berthon P, et al. Oral Uptake of Chlamydia psittaci by Ducklings Results in Systemic Dissemination. PloS one. 2016;11(5):e0154860.

42. Salisch H VMK, Ryll M, Hinz K-H. Chlamydial infections of poultry and human health. World's Poultry Science Journal. 1996;52(3):279-308.

43. Bankowski RA, Mikami T, Kinjo T. Susceptibility of young leghorn chickens to an ornithosis agent isolated from turkeys. The Journal of infectious diseases. 1967;117(2):162-70.

44. Yin L, Lagae S, Kalmar I, Borel N, Pospischil A, Vanrompay D. Pathogenicity of low and highly virulent Chlamydia psittaci isolates for specific-pathogen-free chickens. Avian diseases. 2013;57(2):242-7.

45. Guo W, Li J, Kaltenboeck B, Gong J, Fan W, Wang C. Chlamydia gallinacea, not C. psittaci, is the endemic chlamydial species in chicken (Gallus gallus). Scientific reports. 2016;6:19638.

46. Stokes HS, Martens JM, Chamings A, Walder K, Berg ML, Segal Y, et al. Identification of Chlamydia gallinacea in a parrot and in free-range chickens in Australia. Aust Vet J. 2019;97(10):398-400.

47. Hulin V, Oger S, Vorimore F, Aaziz R, de Barbeyrac B, Berruchon J, et al. Host preference and zoonotic potential of Chlamydia psittaci and C. gallinacea in poultry. Pathogens and disease. 2015;73(1):1-11.

48. Heijne M, van der Goot JA, Fijten H, van der Giessen JW, Kuijt E, Maassen CBM, et al. A cross sectional study on Dutch layer farms to investigate the prevalence and potential risk factors for different Chlamydia species. PloS one. 2018;13(1):e0190774.

49. Donati M, Laroucau K, Guerrini A, Balboni A, Salvatore D, Catelli E, et al. Chlamydiosis in Backyard Chickens (Gallus gallus) in Italy. Vector borne and zoonotic diseases. 2018;18(4):222-5. 
50. Zocevic A, Vorimore F, Marhold C, Horvatek D, Wang D, Slavec B, et al. Molecular characterization of atypical Chlamydia and evidence of their dissemination in different European and Asian chicken flocks by specific real-time PCR. Environ Microbiol. 2012;14(8):2212-22.

51. Szymanska-Czerwinska M, Mitura A, Zareba K, Schnee C, Koncicki A, Niemczuk K. Poultry in Poland as Chlamydiaceae Carrier. J Vet Res. 2017;61(4):411-9.

52. Li L, Luther M, Macklin K, Pugh D, Li J, Zhang J, et al. Chlamydia gallinacea: a widespread emerging Chlamydia agent with zoonotic potential in backyard poultry. Epidemiology and infection. 2017;145(13):2701-3.

53. Ornelas-Eusebio E, Garcia-Espinosa G, Vorimore F, Aaziz R, Durand B, Laroucau K, et al. Crosssectional study on Chlamydiaceae prevalence and associated risk factors on commercial and backyard poultry farms in Mexico. Preventive veterinary medicine. 2020;176:104922.

54. Jeong J, An I, Oem JK, Wang SJ, Kim Y, Shin JH, et al. Molecular prevalence and genotyping of Chlamydia spp. in wild birds from South Korea. The Journal of veterinary medical science. 2017;79(7):1204-9.

55. Frutos MC, Monetti MS, Vaulet LG, Cadario ME, Fermepin MR, Ré VE, et al. Genetic diversity of Chlamydia among captive birds from central Argentina. Avian pathology : journal of the WVPA. 2015;44(1):50-6.

56. Li J, Guo W, Kaltenboeck B, Sachse K, Yang Y, Lu G, et al. Chlamydia pecorum is the endemic intestinal species in cattle while C. gallinacea, C. psittaci and C. pneumoniae associate with sporadic systemic infection. Veterinary microbiology. 2016;193:93-9.

57. Guo W, Jelocnik M, Li J, Sachse K, Polkinghorne A, Pannekoek Y, et al. From genomes to genotypes: molecular epidemiological analysis of Chlamydia gallinacea reveals a high level of genetic diversity for this newly emerging chlamydial pathogen. BMC genomics. 2017;18(1):949.

58. You J, Wu Y, Zhang X, Wang X, Gong J, Zhao Z, et al. Efficient fecal-oral and possible vertical, but not respiratory, transmission of emerging Chlamydia gallinacea in broilers. Veterinary microbiology. 2019;230:90-4.

59. Smit LA, Beer F, Opstal-van Winden AW, Hooiveld M, Beekhuizen J, Wouters IM, et al. Q Fever and pneumonia in an area with a high livestock density: a large population-based study. PloS one. 2012;7.

60. Dickx V, Vanrompay D. Zoonotic transmission of Chlamydia psittaci in a chicken and turkey hatchery. Journal of medical microbiology. 2011;60(Pt 6):775-9.

61. Beeckman DS, Vanrompay DC. Zoonotic Chlamydophila psittaci infections from a clinical perspective. Clinical microbiology and infection : the official publication of the European Society of Clinical Microbiology and Infectious Diseases. 2009;15(1):11-7.

62. Spoorenberg SM, Bos WJ, van Hannen EJ, Dijkstra F, Heddema ER, van Velzen-Blad H, et al. Chlamydia psittaci: a relevant cause of community-acquired pneumonia in two Dutch hospitals. The Netherlands journal of medicine. 2016;74(2):75-81. 

CHAPTER 2 


\section{A cross sectional study on Dutch layer farms to investigate the prevalence and potential risk factors for different Chlamydia species}

Marloes Heijne1, Jeanet A. van der Goot ${ }^{1}$, Helmi Fijten², Joke W. van der Giessen 1,3, Eric Kuijt2, Catharina B.M. Maassen ${ }^{3}$, Annika van Roon ${ }^{3}$, Ben Wit ${ }^{4}$, Ad P. Koets ${ }^{1,5}$, Hendrik I.J. Roest ${ }^{1}$

${ }^{1}$ Department of Bacteriology and Epidemiology, Wageningen Bioveterinary Research, Lelystad, the Netherlands

${ }^{2}$ Department of Infection Biology, Wageningen Bioveterinary Research, Lelystad, the Netherlands

${ }^{3}$ Center for Zoonoses and Environmental Microbiology, National Institute for Public Health and the Environment (RIVM), Bilthoven, the Netherlands

${ }^{4}$ Consumer and Safety Division, Food and Consumer Product Safety Authority (NVWA), Utrecht, the Netherlands

${ }^{5}$ Department of Farm Animal Health, Faculty of Veterinary Medicine, Utrecht University, Utrecht, the Netherlands

PLoS One. 2018 Jan 11;13(1):e0190774. doi: 10.1371/journal.pone.0190774. PMID: 29324884; PMCID: PMC5764275. 


\section{Abstract}

In poultry several Chlamydia species have been detected, but Chlamydia psittaci and Chlamydia gallinacea appear to be most prevalent and important. Chlamydia psittaci is a well-known zoonosis and is considered to be a pathogen of poultry. Chlamydia gallinacea has been described more recently. Its avian pathogenicity and zoonotic potential have to be further elucidated. Within the Netherlands no data were available on the presence of Chlamydia on poultry farms. As part of a surveillance programme for zoonotic pathogens in farm animals, we investigated pooled faecal samples from 151 randomly selected layer farms. On a voluntary base, 69 farmers, family members or farm workers from these 151 farms submitted a throat swab. All samples were tested with a generic 23S Chlamydiaceae PCR followed by a species specific PCR for C. avium, C. gallinacea and C. psittaci. C. avium and psittaci DNA was not detected at any of the farms. At 71 farms the positive result could be confirmed as $C$. gallinacea. Variables significantly associated with the presence of $C$. gallinacea in a final multivariable model were 'age of hens', 'use of bedding material' and 'the presence of horses'. The presence of $C$. gallinacea was associated with neither clinical signs, varying from respiratory symptoms, nasal and ocular discharges to diarrhoea, nor with a higher mortality rate the day before the visit. All throat swabs from farmers, family members or farm workers tested negative for Chlamydia DNA, giving no further indication for possible bird-to-human (or human-to-bird) transmission. 


\section{Introduction}

Chlamydia avium, Chlamydia gallinacea and Chlamydia psittaci belong to the family of Chlamydiaceae, a group of obligate intracellular bacteria. Chlamyia psittaci is widespread and can infect over 465 bird species and several mammalian species, including humans (1). Pathogenicity in animals depends on host species and C. psittaci strain. Clinical symptoms in birds vary from asymptomatic to acute death. Chlamydia psittaci is a wellknown zoonosis and the cause of psittacosis. Transmission from birds to humans occurs via aerosolised respiratory or faecal excretions. In the Netherlands, psittacosis is notifiable in humans and pet birds but not in poultry. In poultry, chickens appeared to be less sensitive to chlamydial infection and a sporadic source of human infection (1-3). However, in recent publications $C$. psittaci is regularly detected and chicken-to-human transmission is more frequently described (4-6).

Chlamydia avium and C. gallinacea have been detected in pet birds and poultry since 2009, first being classified as "atypical" and in 2014 added as new members of the genus Chlamydia (7-9). Chlamydia avium has been found in psittacines and pigeons, C. gallinacea in chickens, guinea fowl and turkeys (10). Recent studies hypothesised C. gallinacea to be endemic in chickens causing only mild clinical signs such as reduced weight gain in broilers (11). Its zoonotic potential was suggested, but conclusive evidence has not been presented yet (8).

The impact of transmission of zoonotic pathogens from farm animals to humans was highlighted by the Dutch Q fever outbreak (2007-2010). Due to this outbreak, studies were initiated to assess the public health risks of intensive farming in densely populated areas (12). One of the findings was a higher incidence of human pneumonia cases in the direct proximity of poultry farms $(13,14)$. The cause of this higher incidence was unknown. We therefore hypothesised that C. psittaci or C. gallinacea could play a role. However, no data were available on the presence of $C$. psittaci and $C$ gallinacea on Dutch poultry farms.

We investigated 755 faecal samples from 151 layer farms for the presence of Chlamydiacea DNA. Per farm a questionnaire was completed to identify possible risk factors. To gather information on possible bird to human transmission, farmers, family members or farm workers were invited to participate on a voluntary basis in throat swab sampling. 


\section{Materials and methods}

\section{Sampling strategy}

Between March 2015 and January 2016, a cross-sectional study on layer farms was performed as part of a surveillance programme for zoonotic pathogens in farm animals. From the 993 layer farms in the Netherlands, 154 farms were randomly selected, stratified on farming system (conventional $n=79$, free range $n=34$, organic $n=22$, enriched cages $\mathrm{n}=8$, enriched colony $\mathrm{n}=6$ ). Finally, 151 farms completed a questionnaire and were included in the analysis. For Chlamydia testing, five pooled faecal samples were collected from one barn per farm, resulting in 755 samples. Each pooled sample contained twelve scoops of fresh faeces. Additional information on farm characteristics, husbandry practices, biosecurity measures, clinical history and antibiotic usage was acquired via a questionnaire (Fig. 1). Farmers, family members and farm workers were asked to participate in the poultry-to-human transmission study by submitting two throat swabs (collected through self-sampling) for Chlamydia testing. In total 69 farmers, family members or farm workers from 41 farms participated in the study.

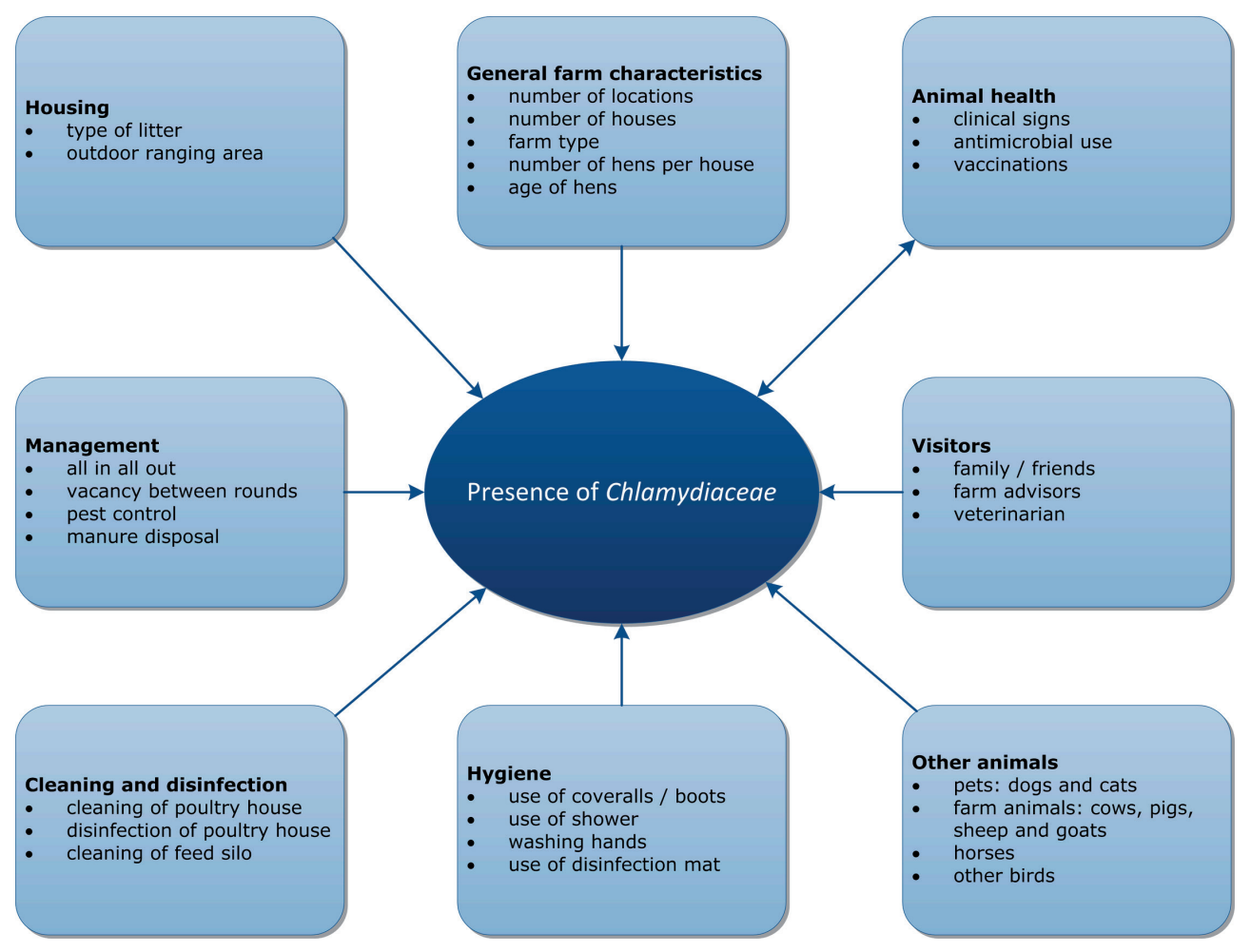

Fig. 1. Overview of risk factors

Overview of possible risk factors for the presence of Chlamydiaceae on which information was gathered via a questionnaire. 


\section{Ethics statement}

The Medical Ethics Review Committee (Utrecht Medical Centre, Utrecht) stated that the Medical Research Involving Human Subjects Act (WMO) does not apply to this study and therefore no official approval for the study is required under the WMO. All volunteers gave their written consent for participation in the study.

\section{Laboratory tests}

DNA isolation of all pooled faecal samples was performed with a NucliSENS ${ }^{\circledR}$ easyMAG ${ }^{\circledR}$ (Biomerieux, Zaltbommel, the Netherlands). In brief, faecal material was taken from each sample with a dry swab, suspended in $1.5 \mathrm{ml}$ Phosphate Buffered Saline (PBS) and thoroughly vortexed. From this suspension, $500 \mu \mathrm{l}$ was added to $2 \mathrm{ml}$ NucliSENS ${ }^{\circledR}$ lysis buffer for off-board lysis. After at least one hour of incubation at room temperature, the lysis buffer was added to $80 \mu$ of silica and extracted according to manufacturer instructions for specific protocol B. Within this protocol an optimised washing protocol is used with extra and longer washing steps. The final elution volume was $100 \mu$ l. DNA isolation of human throat swabs was performed with a MagNA Pure ${ }^{\circledR}$ LC (Roche Diagnostics, Almere, The Netherlands) according to manufacturer instructions for off-board lysis. Of the sample $200 \mu \mathrm{l}$ was processed to a final elution volume of $50 \mu \mathrm{l}$. Chlamydiaceae-DNA was detected using a generic PCR that targeted the 23S rRNA gene with primers and probes according to Ehricht et al (15). Chlamydia psittaci DNA was detected using a PCR that targeted the ompA gene with primers and probes according to Pantchev et al (16). For C. avium and C. gallinacea a duplex PCR was used targeting the enoA gene. For $C$. avium primer and probe sequences were used according to Zocevic et al (9). For C. gallinacea primer and probe sequences were used according to Laroucau et al (6). To validate the C. avium and C. gallinacea duplex PCR, 10-fold serial dilutions (single and in a mixture) of $C$. gallinacea strain 14DC0101 and C. avium strain 10DC97 were tested. The duplex PCR appeared to be as sensitive as the single PCR. No differences in Ct values were observed when C. avium and $C$. gallinacea were added in a single dilution or as a mixture. The final volume of the reaction mixture was $20 \mu \mathrm{l}$, including $5 \mu$ l of the DNA template, $10 \mu$ ITaqMan ${ }^{\circledR}$ Fast Universal PCR Master Mix (Applied Biosystems, Fisher Scientific, Landsmeer, the Netherlands), $1 \mu \mathrm{M}$ of each primer, $0.2 \mu \mathrm{M}$ of the probes, $0.2 \mu \mathrm{I}$ UDG $(5 \mathrm{U} / \mu \mathrm{l})$ and distilled PCR water to reach the final volume. Amplification was carried out in an ABI 7500 Fast Real-Time PCR system (Applied Biosystems, Fisher Scientific, Landsmeer, the Netherlands) using the following cycling parameters: 5 min at $37 \circ \mathrm{C}, 20 \mathrm{sec}$ at $95 \circ \mathrm{C}, 50$ cycles of $95 \circ \mathrm{C}$ for $3 \mathrm{sec}$ and $60 \circ \mathrm{C}$ for $30 \mathrm{sec}$. As a control for DNA extraction, a known C. psittaci positive faecal swab was used. In each $23 S$ and $C$. psittaci PCR run a dilution series of three $C$. psittaci DNA isolates was used as positive controls. In the $C$. gallinacea and $C$. avium duplex PCR, DNA from $C$. gallinacea strain 14DC0101 and C. avium 10DC97 and a mix of both strains were used as positive controls. Each real-time PCR run included a non-template control using $5 \mu \mathrm{l}$ distilled water as template, and during the extraction per 12 samples a negative sample 
with $1.5 \mathrm{ml}$ PBS was added. Samples with a $\mathrm{Ct}$ value up to 40 were considered positive and samples with a $\mathrm{Ct}$ value above 40 were considered negative. Farms were considered positive if at least one of five samples tested positive in the PCR.

\section{GIS map}

Chlamydia gallinacea positive and negative farms were plotted on a laying hen density map of the Netherlands (Fig. 2). Data were extracted from CBS Statline (http://statline.cbs. $\mathrm{nl}$ ) and imported into QGIS version 2.18.

\section{Statistical analyses}

Farm prevalence was determined with an exact (Clopper-Pearson) 95 percent confidence interval (epitools.ausvet.com.au). Data from the questionnaires were collected via a digital form in Epi Info ${ }^{\mathrm{TM}}$ and analysed using IBM SPSS Statistics for Windows, Version 23.0 (IBM Corp., Armonk, N.Y., USA). Overlapping variables or small categories were merged or summarised when possible. Potential risk factors for the presence of $C$. gallinacea were initially examined with a univariable analysis using a Chi square test or a logistic regression for continuous variables. Variables associated $(p \leq 0.20)$ with the outcome of interest (presence of $C$. gallinacea) were considered for inclusion in a stepwise, backward, multiple logistic regression analysis. The selected variables for the multivariable analysis were tested for mutual correlation. A likelihood ratio test was performed to eliminate variables from the multivariable model. Variables had to be significant $(p \leq 0.05)$ to remain in the final model. The goodness of fit of the final model was tested using the Hosmer and Lemeshow test.

\section{Results}

Chlamydiaceae DNA was detected on 74 of the 151 farms and confirmed as C. gallinacea on 71 farms (farm prevalence 47\%, 95\% Cl: 39-55\%). Neither C. psittaci DNA nor C. avium DNA was detected in any of the samples from the 151 farms. The distribution of the number of positive samples per farm in the 235 Chlamydiaceae PCR and the C. gallinacea PCR is shown in Table 1. On 31 farms all five samples were positive in both the Chlamydiaceae PCR and C. gallinacea PCR, whereas on 67 farms all five samples were negative in both the Chlamydiaceae PCR and C. gallinacea PCR. At seven farms no Chlamydiaceae DNA was detected, but per farm one or two samples tested positive for C. gallinacea DNA with $\mathrm{Ct}$ values above 36. The 71 farms that had one or more positive samples in both the Chlamydiaceae PCR and the C. gallinacea PCR were included in the risk factor analysis. 
Table 1. The distribution of the number of positive samples per farm in the Chlamydiaceae and C. gallinacea PCR

\begin{tabular}{|c|c|c|c|c|c|c|c|c|}
\hline & & \multicolumn{7}{|c|}{$\begin{array}{l}\text { Number of positive samples per farm in C. gallinacea } P C R \\
(n=5 \text { per farm) }\end{array}$} \\
\hline & & 0 & 1 & 2 & 3 & 4 & 5 & total \\
\hline \multirow{7}{*}{$\begin{array}{c}\text { Number } \\
\text { of positive } \\
\text { samples } \\
\text { per farm in } \\
\text { Chlamydiaceae } \\
\text { PCR (n=5 per } \\
\text { farm) }\end{array}$} & 0 & 67 & 6 & 1 & 0 & 0 & 0 & 74 \\
\hline & 1 & 2 & 6 & 1 & 1 & 0 & 0 & 10 \\
\hline & 2 & 2 & 3 & 1 & 2 & 2 & 0 & 11 \\
\hline & 3 & 0 & 2 & 1 & 3 & 0 & 1 & 7 \\
\hline & 4 & 0 & 0 & 2 & 1 & 3 & 1 & 6 \\
\hline & 5 & 0 & 0 & 0 & 1 & 3 & 30 & 34 \\
\hline & total & 71 & 17 & 6 & 8 & 8 & 32 & $142^{*}$ \\
\hline
\end{tabular}

* The results of 142 farms are shown. From seven farms one or more samples showed inhibition. From two farms only four samples could be tested. The results of these nine farms are not shown in the table, but the farm level results were used in the analysis.

The location of the positive and negative farms is shown in Fig. 2. Chlamydia gallinacea positive and negative farms appear to be equally distributed in the Netherlands. 


\section{Legend}

- farm positive for C. gallinacea

- farm negative for C. gallinacea

Layer hens per municipality
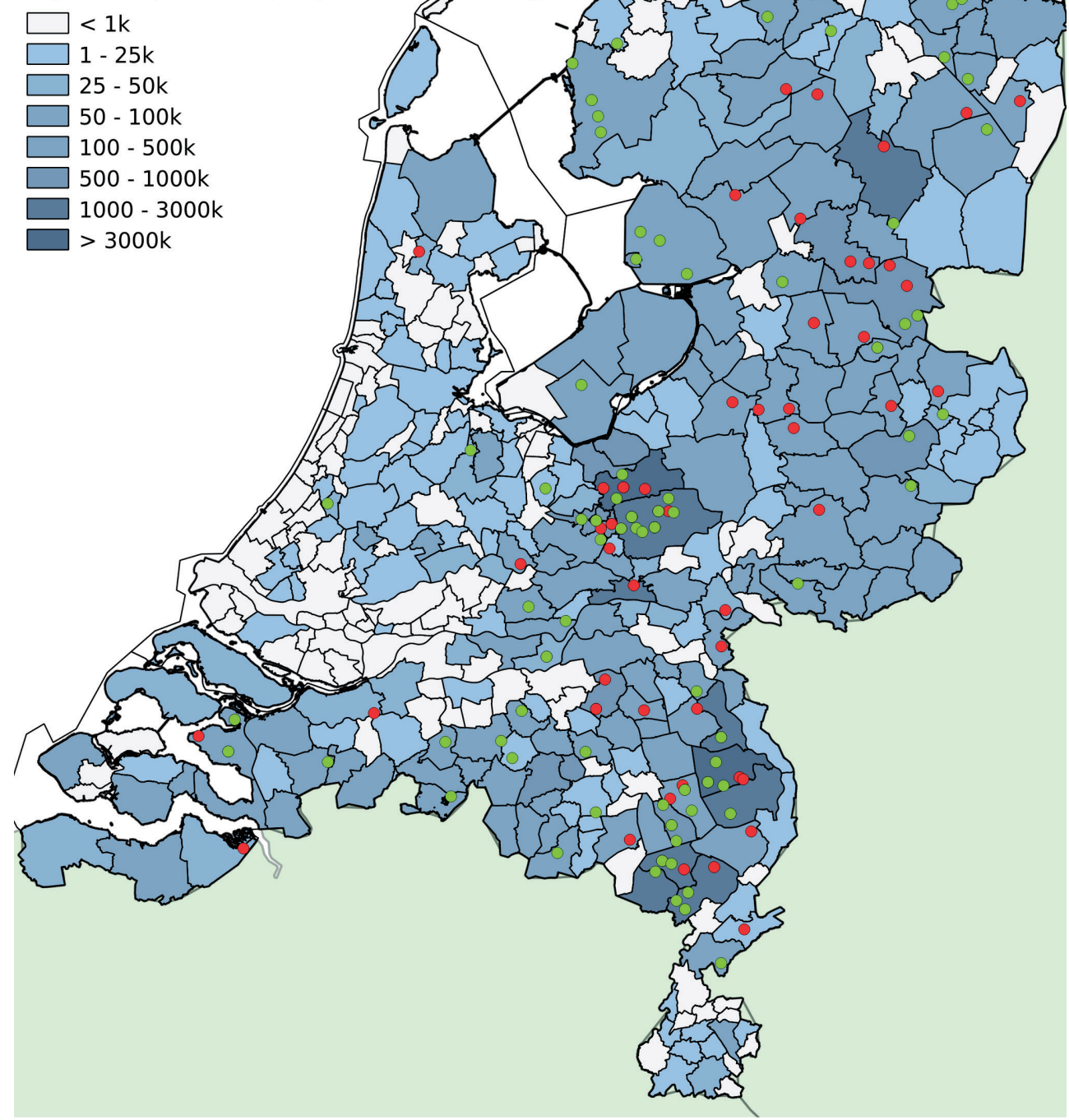

Fig. 2. Map with C. gallinacea positive and negative farms

Chlamydia gallinacea positive and negative farms plotted on a laying hen density map of the Netherlands. 
General descriptors about farm type and the median farm size are shown in Table 2. Farm type is related to farm size. Farms with enriched cages and colony systems are larger than free range and organic farms. Due to the relation with farm type, farm size was excluded from the analysis. Background and coding information on the variables in the univariable and multivariable analyses are added in the S1 and S2 files. For the variables 'age of hens' and 'manure disposal' the smallest categories were merged. From the variable 'vacancy period' (period between two flocks, when the barn is empty), outliers with a vacancy period above 90 days were excluded from the analysis.

In the univariable analysis, ten variables met the criteria of $p \leq 0.2$, i.e. 'age of hens', 'use of bedding material', 'presence of horses', 'frequency of manure disposal', 'visitors have to shower before entrance', 'other birds', 'free range', 'vaccination against Pasteurella multocida' or 'Egg Drop Syndrome' and 'vacancy period' (Table 3). No mutual correlations were found between these ten variables and they were all included in the multivariable analysis. No associations were found between the presence of $C$. gallinacea and 'one or more locations,' 'more than one poultry house', 'all in all out at farm level', 'fly control', 'visitors', 'disinfection method', 'frequency of cleaning of the feed silo', 'washing hands before entrance' and the 'presence of other farm animals or pets'. All farms reported that they controlled rats and mice. The variables 'use of disinfection mat before entrance' and 'use of tools in one or more houses' were not included in the analysis, due to inconsistent answers in the questionnaires.

In the multivariable analysis, three variables were significantly associated with the presence of C. gallinacea as shown in Table 4: 'age of hens,' 'use of bedding material' and 'presence of horses. The final model met the criteria of the Hosmer-Lemeshow goodness of fit test.

Table 2. General descriptors of farm type and farm size

\begin{tabular}{lccc}
\hline Farm type & $\begin{array}{c}\text { Number of } \\
\text { farms }\end{array}$ & $\begin{array}{c}\text { \% of participating } \\
\text { farms }\end{array}$ & $\begin{array}{c}\text { Median farm size } \\
\text { (range) }\end{array}$ \\
\hline Conventional (Barn egg) & 79 & 52.3 & $33,696(1,000-239,000)$ \\
\hline Free range & 34 & 22.8 & $24,410(900-117,000)$ \\
\hline Enriched cages & 8 & 5.3 & $97,693(648-180,000)$ \\
\hline Enriched colony system & 6 & 3.9 & $182,600(66,000-383,000)$ \\
\hline Organic & 22 & 14.5 & $11850(500-32,800)$ \\
\hline Missing information & 2 & 1.3 & n.a. \\
\hline Total & 151 & 100 & $28,750(500-383,000)$ \\
\hline
\end{tabular}


Table 3. Variables from the univariable analysis with $p \leq 0.2$ (ranked by $p$-value)

\begin{tabular}{|c|c|c|c|c|}
\hline Variable & $\begin{array}{c}\text { No. of infec- } \\
\text { ted farms } \\
n=71 *(\%)\end{array}$ & $\begin{array}{c}\text { No. of non- } \\
\text { infected farms } \\
n=80(\%)\end{array}$ & $\begin{array}{l}\text { Odds Ratio } \\
\text { (Cl 95\%) }\end{array}$ & $\begin{array}{c}\text { p-value } \\
\text { (Chi square) }\end{array}$ \\
\hline Age of hens ${ }^{\#}$ & & & & $<0.01$ \\
\hline till 40 weeks & $15 / 70(21.4)$ & $32 / 77(41.6)$ & $\operatorname{Ref}$ & $\operatorname{Ref}$ \\
\hline $40-60$ weeks & $28 / 70(40.0)$ & 13/77 (16.9) & $4.6(1.87-11.29)$ & $<0.01$ \\
\hline older than 60 weeks & $27 / 70(38.6)$ & $32 / 77(41.6)$ & $1.80(0.81-4.00)$ & 0.15 \\
\hline Use of bedding material & $64 / 69(92.8)$ & $60 / 80(75.0)$ & $4.27(1.51-12.09)$ & $<0.01$ \\
\hline Horses present & $26 / 71(36.6)$ & $14 / 80(17.5)$ & $2.72(1.28-5.78)$ & $<0.01$ \\
\hline Manure disposal ${ }^{\#}$ & & & & 0.08 \\
\hline once or less than once a week & $6 / 70(8.6)$ & $18 / 77(23.4)$ & Ref & Ref \\
\hline once every two weeks & $21 / 70(29.6)$ & $14 / 77(18.3)$ & $4.50(1.43-14.14)$ & 0.10 \\
\hline once a month & $17 / 70(23.9)$ & $18 / 77(23.4)$ & $2.83(0.91-8.83)$ & 0.07 \\
\hline less than once a month & $26 / 70(38.0)$ & $27 / 77(35.1)$ & $8.89(0.99-8.4)$ & 0.05 \\
\hline $\begin{array}{l}\text { Use of shower before entrance } \\
\text { (visitors) }\end{array}$ & $6 / 71(8.5)$ & $14 / 80(17.5)$ & $0.44(0.16-1.16)$ & 0.10 \\
\hline Other birds present ${ }^{i}$ & $5 / 71(7.0)$ & $1 / 80(1.3)$ & $5.99(0.68-52.5)$ & 0.10 \\
\hline $\begin{array}{l}\text { Vaccination against Pasteurella } \\
\text { multocida' }\end{array}$ & $8 / 71(11.3)$ & $3 / 80(3.8)$ & $3.26(0.83-12.80)$ & 0.12 \\
\hline Vacancy periodii & & & / & 0.14 \\
\hline $\begin{array}{l}\text { Vaccination against Egg Drop } \\
\text { Syndrome }\end{array}$ & $41 / 71(57.7)$ & $37 / 80(46.3)$ & $1.59(0.83-3.02)$ & 0.16 \\
\hline Free range sampled house & $39 / 69(42.0)$ & $25 / 80(31.3)$ & $1.60(0.81-3.12)$ & 0.17 \\
\hline
\end{tabular}

${ }^{*}$ Due to missing values, the number of farms per variable can differ, ${ }^{*} p$-value was calculated with logistic regression, 'iFisher exact $p$-value was used (cells with counts $n<5$ ), "icontinuous variable 
Table 4. Results of multivariable analysis (ranked by p-value)

\begin{tabular}{lcc}
\hline Variable & Odds Ratio (CI 95\%) & p-value \\
\hline Age of hens & ref & $<0.01$ \\
\hline till 40 weeks & $5.41(2.02-14.53)$ & ref \\
\hline 40-60 weeks & $2.28(0.94-5.53)$ & $<0.01$ \\
\hline older than 60 weeks & $4.22(1.40-12.75)$ & 0.07 \\
\hline Use of bedding material & $2.67(1.16-6.12)$ & 0.01 \\
\hline Horses present & & 0.02 \\
\hline
\end{tabular}

For the multivariable analysis 139 farms were selected, 12 had missing values for one or more of the selected variables.

No associations between the presence of C. gallinacea DNA and clinical signs, varying from respiratory symptoms and nasal and ocular discharges to diarrhoea, were found. The number of farms reporting clinical signs was low $(n=11)$. Also no association was found between the mortality rate the day before the visit and the presence of $C$. gallinacea. $A$ total of 83 farms reported a mortality rate per day of $<0.01 \%, 58$ a mortality rate between $0.01 \%$ and $0.05 \%$, and 7 farms a mortality rate $>0.05 \%$ ( 3 farms did not report the mortality rate the day before the visit).

The 69 human throat swabs all tested negative in the Chlamydiaceae PCR. A total of 26 human samples were collected from farmers, family members or workers from $17 \mathrm{C}$. gallinacea DNA positive farms and 42 samples from 24 C. gallinacea DNA negative farms. One human sample could not be related to a sampled farm.

In summary, C. gallinacea DNA is highly prevalent on Dutch layer farms (farm prevalence 47\%, 95\% Cl 39-55\%), while neither C. psittaci DNA nor C. avium DNA were detected in any of the samples from the 151 farms. In the multivariable model, the presence of $C$. gallinacea appears to be associated with the ' age of hens,' presence of horses' and 'use of bedding material'. No association was found with clinical signs or mortality rate the day before the visit. All of the 69 human throat swabs collected from farmers, family members or workers tested negative for Chlamydiaceae DNA.

\section{Discussion}

Our cross-sectional study shows that C. gallinacea DNA is present on $47 \%$ (95\% Cl 39-55\%) of layer farms in the Netherlands. The high prevalence of $C$. gallinacea DNA is in agreement with publications that postulate C. gallinacea to be the most important Chlamydia spp in chickens $(11,17)$. In 2012, Zocevic et al. detected mainly DNA of atypical Chlamydias (later 
redefined as C. gallinacea) in 95 of 283 samples from different poultry flocks from France, Greece, Slovenia, Croatia and China (9). Guo et al. detected C. gallinacea DNA in about $20 \%(359 / 1791)$ of oral and cloacal swabs of chickens from different provinces in China (11). Hulin et al. reported a predominance of $C$. gallinacea in a poultry slaughterhouse where mainly chickens were slaughtered; in 52 / 129 flocks one or more samples were PCR positive for C. gallinacea.

C. psittaci and C. avium DNA were not detected at any of the 151 farms (95\% Cl 0- 2\%). These results are in line with the findings of Guo et al., where 41 of 1791 (2.3\%) chicken samples were PCR positive for C. psittaci (11), and with the study of Hulin et al. where only one of the 129 flocks (bird species not specified) from the chicken slaughterhouse was PCR positive (17). In contrast, Lagae et al. PCR detected and cultured C. psittaci from individual pharynx swabs from $7 / 7$ broiler, $5 / 5$ layer and 6/7 broiler breeder farms in Belgium. Differences in sampling methods might play a role. It has been shown that pharyngeal swabs are a more sensitive sampling method than cloacal swabs or faecal samples for the detection of $C$. psittaci (18). Culturing, however has proven to be a less sensitive detection method than PCR, so this might not fully explain the large difference in prevalence (19). The prevalence of $C$. psittaci might differ between countries. The absence of $C$. avium was expected. So far, this bacterium has only been found in psittacines and pigeons and not in poultry (7).

In the risk factor analysis ' age of hens', 'use of bedding material' and 'presence of horses' were associated with the presence of $C$. gallinacea. The age related risk for the presence of $C$. gallinacea peaks between 40 and 60 weeks (OR 5.41, $p<0.01$ ). Factors that might influence this risk are the moment of introduction, the duration of $C$. gallinacea infections and the acquisition of immunity. However, this information is currently not available for C. gallinacea infections. Studies with a more longitudinal approach are therefore needed. The association with the 'use of bedding material' might be explained by the introduction of the bacterium via bedding material or the effect of this material on the persistence of the bacterium in the environment. It has been reported that the elementary bodies of other Chlamydiaceae can survive in litter for several months (20). There is no obvious explanation for the association with the 'presence of horses'. Several Chlamydia species have been detected in horses, but the presence of $C$. gallinacea has not been described (21). However, C. gallinacea has been detected in vaginal swabs from cattle in China suggesting it might not be restricted to poultry (22). There might be other associated factors as well, such as frequent movement of trailers, which explains the association with horses. More detailed studies are needed to confirm the relation between the risk factors in the final model and the presence of $C$. gallinacea DNA. 
We did not observe an association between the presence of C. gallinacea and 'clinical signs', based on the results of the questionnaire. It should be noted that only 11 farms reported overt clinical problems, which varied from respiratory symptoms and nasal and ocular discharges to diarrhoea. Also no association was found with the mortality rate the day before the visit. An association with increased mortality cannot be excluded, because the mortality rate might have increased earlier in the infection and subsequently returned to a normal level. To study this we should have analysed for a period longer than 1 day before the visit. Furthermore, a possible clinical outcome of a C. gallinacea infection could be more subtle or subclinical. For example Guo et al. did not report any clinical signs, but did find a reduction in growth of broiler chicks (11). Reinhold et al. discussed the role of Chlamydiaceae in cattle and suggested subclinical and chronic chlamydial infections might be economically more important than a clinical outbreak (20). Further studies should also take into account subclinical or more economically important parameters, such as egg production during the entire production round.

All human samples collected, tested negative for Chlamydiacea DNA. Participants were not selected for clinical signs and 26 were working or living at a C. gallinacea positive farm. A positive sample would have given an indication of possible bird-to-human (or humanto-bird) transmission. To date C. gallinacea has only been suggested as a cause of human pneumonia (8), but in our study we could not confirm this. Sputum or bronchoalveolar lavage fluid (BAL) from patients with community acquired pneumonia (CAP) should be examined to further investigate whether $C$. gallinacea could be a cause of human pneumonia.

Our study adds to the hypothesis that C. gallinacea is the endemic Chlamydia of chickens. However, many questions still need to be answered. The most important of these is to elucidate the zoonotic potential of C. gallinacea and to investigate the pathogenesis of a C. gallinacea infection, as these could be of economic significance for the poultry sector.

\section{Funding}

This study was partly financed by the Food and Consumer Product Safety Authority (NVWA) and partly as a public-private-partnership: "1H4F-Chlamydia and respiratory problems in poultry" (TKI-AF-14212, http://www.1 health4food.nl/nl/show/Chlamydia-enrespiratoire-problemen-in-pluimvee-.htm). 


\section{Acknowledgements}

The authors thank Christiane Schnee from the FLI in Jena, Germany for providing strains of C. avium (12DC97) and C. gallinacea (14DC0101 and 08DC00063). The authors acknowledge Herma Buys and Evelien Kern-van Nes of the WBVR diagnostic lab for their assistance in the DNA isolation of the faecal samples from the layers and Pieter Overduin from the National Institute for Public Health and the Environment for the DNA isolation of the human throat swabs.

\section{Supporting information}

S1 File. Background data risk factor analysis. This file holds data on the variables used in the univariable and multivariable analysis and can be found at: https://doi.org/10.1371/ journal.pone.0190774.s001

S2 File. Variable coding information. This file holds information on the coding of the variables and can be found at: https://doi.org/10.1371/journal.pone.0190774.s002 


\section{References}

1. Kaleta EF, Taday EM. Avian host range of Chlamydophila spp. based on isolation, antigen detection and serology. Avian pathology : journal of the WVPA. 2003;32(5):435-61.

2. Suwa T, Ando S, Hashimoto N, Itakura C. Pathology of experimental chlamydiosis in chicks. Nihon juigaku zasshi The Japanese journal of veterinary science. 1990;52(2):275-83.

3. Salisch H VMK, Ryll M, Hinz K-H. Chlamydial infections of poultry and human health. World's Poultry Science Journal. 1996;52(3):279-308.

4. Lagae S, Kalmar I, Laroucau K, Vorimore F, Vanrompay D. Emerging Chlamydia psittaci infections in chickens and examination of transmission to humans. Journal of medical microbiology. 2014;63(Pt 3):399-407.

5. Yin L, Kalmar ID, Lagae S, Vandendriessche S, Vanderhaeghen W, Butaye P, et al. Emerging Chlamydia psittaci infections in the chicken industry and pathology of Chlamydia psittaci genotype $B$ and $D$ strains in specific pathogen free chickens. Veterinary microbiology. 2013;162(2-4):740-9.

6. Laroucau K, Aaziz R, Meurice L, Servas V, Chossat I, Royer H, et al. Outbreak of psittacosis in a group of women exposed to Chlamydia psittaci-infected chickens. Euro surveillance : bulletin Europeen sur les maladies transmissibles = European communicable disease bulletin. 2015;20(24).

7. Sachse K, Laroucau K, Riege K, Wehner S, Dilcher M, Creasy HH, et al. Evidence for the existence of two new members of the family Chlamydiaceae and proposal of Chlamydia avium sp. nov. and Chlamydia gallinacea sp. nov. Systematic and applied microbiology. 2014;37(2):79-88.

8. Laroucau K, Vorimore F, Aaziz R, Berndt A, Schubert E, Sachse K. Isolation of a new chlamydial agent from infected domestic poultry coincided with cases of atypical pneumonia among slaughterhouse workers in France. Infection, genetics and evolution : journal of molecular epidemiology and evolutionary genetics in infectious diseases. 2009;9(6):1240-7.

9. Zocevic A, Vorimore F, Vicari N, Gasparini J, Jacquin L, Sachse K, et al. A real-time PCR assay for the detection of atypical strains of Chlamydiaceae from pigeons. PloS one. 2013;8(3):e58741.

10. Sachse K, Laroucau K, Vanrompay D. Avian Chlamydiosis. Current Clinical Microbiology Reports. 2015;2(1):10-21.

11. Guo W, Li J, Kaltenboeck B, Gong J, Fan W, Wang C. Chlamydia gallinacea, not C. psittaci, is the endemic chlamydial species in chicken (Gallus gallus). Scientific reports. 2016;6:19638.

12. Roest HI, Tilburg JJ, van der Hoek W, Vellema P, van Zijderveld FG, Klaassen $\mathrm{CH}$, et al. The Q fever epidemic in The Netherlands: history, onset, response and reflection. Epidemiology and infection. 2011;139(1):1-12.

13. Smit LA, van der Sman-de Beer F, Opstal-van Winden AW, Hooiveld M, Beekhuizen J, Wouters IM, et al. $\mathrm{Q}$ fever and pneumonia in an area with a high livestock density: a large population-based study. PloS one. 2012;7(6):e38843. 
14. Freidl GS, Spruijt IT, Borlee F, Smit LA, van Gageldonk-Lafeber AB, Heederik DJ, et al. Livestockassociated risk factors for pneumonia in an area of intensive animal farming in the Netherlands. PloS one. 2017;12(3):e0174796.

15. Ehricht R, Slickers P, Goellner S, Hotzel H, Sachse K. Optimized DNA microarray assay allows detection and genotyping of single PCR-amplifiable target copies. Molecular and cellular probes. 2006;20(1):60-3.

16. Pantchev A, Sting R, Bauerfeind R, Tyczka J, Sachse K. New real-time PCR tests for speciesspecific detection of Chlamydophila psittaci and Chlamydophila abortus from tissue samples. Veterinary journal. 2009;181(2):145-50.

17. Hulin V, Oger S, Vorimore F, Aaziz R, de Barbeyrac B, Berruchon J, et al. Host preference and zoonotic potential of Chlamydia psittaci and C. gallinacea in poultry. Pathogens and disease. 2015;73(1):1-11.

18. Andersen AA. Comparison of pharyngeal, fecal, and cloacal samples for the isolation of Chlamydia psittaci from experimentally infected cockatiels and turkeys. Journal of veterinary diagnostic investigation : official publication of the American Association of Veterinary Laboratory Diagnosticians, Inc. 1996;8(4):448-50.

19. Hewinson RG, Griffiths PC, Bevan BJ, Kirwan SE, Field ME, Woodward MJ, et al. Detection of Chlamydia psittaci DNA in avian clinical samples by polymerase chain reaction. Veterinary microbiology. 1997;54(2):155-66.

20. Reinhold P, Sachse K, Kaltenboeck B. Chlamydiaceae in cattle: commensals, trigger organisms, or pathogens? Veterinary journal. 2011;189(3):257-67.

21. Pantchev A, Sting R, Bauerfeind R, Tyczka J, Sachse K. Detection of all Chlamydophila and Chlamydia spp. of veterinary interest using species-specific real-time PCR assays. Comparative immunology, microbiology and infectious diseases. 2010;33(6):473-84.

22. Li J, Guo W, Kaltenboeck B, Sachse K, Yang Y, Lu G, et al. Chlamydia pecorum is the endemic intestinal species in cattle while C. gallinacea, C. psittaci and C. pneumoniae associate with sporadic systemic infection. Veterinary microbiology. 2016;193:93-9. 

CHAPTER 3

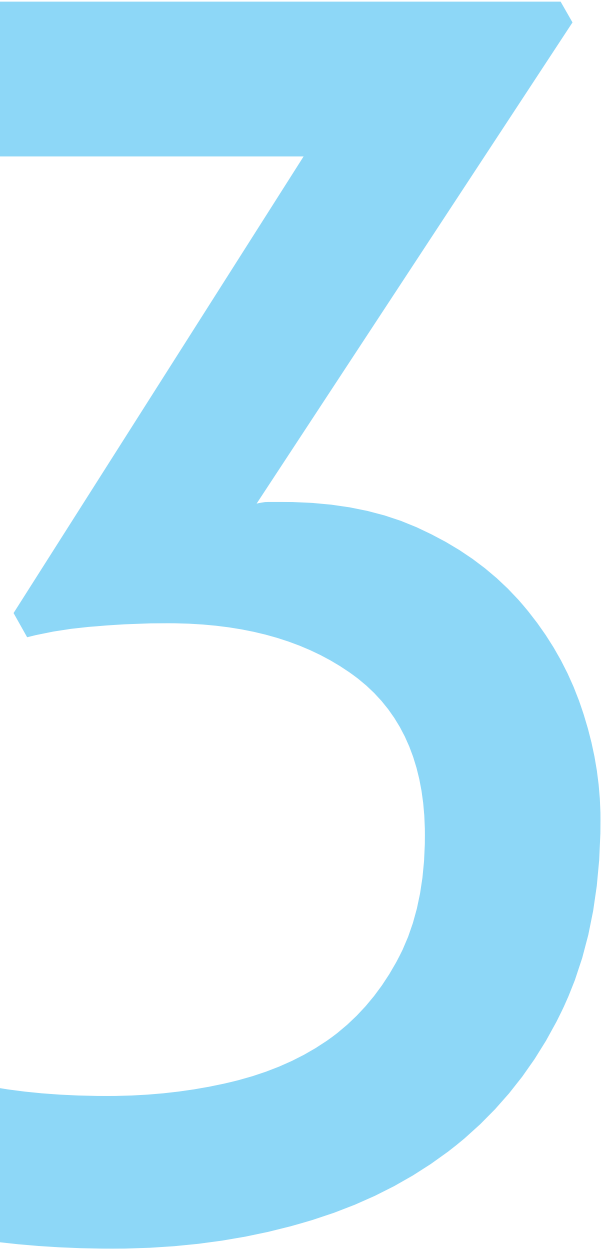




\section{Genetic and phenotypic analysis of the pathogenic potential of two novel Chlamydia gallinacea strains compared to Chlamydia psittaci}

Marloes Heijne ${ }^{1 *}$, Martina Jelocnik², Alexander Umanets ${ }^{1}$, Michael S.M. Brouwer ${ }^{1}$, Annemieke Dinkla', Frank Harders ${ }^{3}$, Lucien J.M. van Keulen ${ }^{3}$, Hendrik Jan Roest ${ }^{1, \#}$, Famke Schaafsma ${ }^{1, \# \text {, }}$ Francisca C. Velkers ${ }^{4}$, Jeanet A. van der Goot ${ }^{5}$, Yvonne Pannekoek ${ }^{6}$ and Ad P. Koets ${ }^{1,4}$

${ }^{1}$ Department of Bacteriology and Epidemiology, Wageningen Bioveterinary Research, Lelystad, the Netherlands

${ }^{2}$ Genecology Research Centre, University of the Sunshine Coast, Sippy Downs, Australia

${ }^{3}$ Department of Infection Biology, Wageningen Bioveterinary Research, Lelystad, the Netherlands

${ }^{4}$ Department of Population Health Sciences, Faculty of Veterinary Medicine, Utrecht University, Utrecht, the Netherlands

${ }^{5}$ Department of Diagnostics and Crisis Organisation, Wageningen Bioveterinary Research, Lelystad, the Netherlands

${ }^{6}$ Department of Medical Microbiology, Amsterdam UMC, University of Amsterdam, the Netherlands

\# Current affiliation: Hendrik Jan Roest, Directorate Animal Supply Chain and Animal Welfare, Ministry of Agriculture, Nature and Food Quality, The Hague, the Netherlands

\# Current affiliation: Famke Schaafsma, Department of Healthy Living, TNO, Zeist, the Netherlands

Sci Rep. 2021 Aug 13;11(1):16516. doi: 10.1038/s41598-021-95966-9. PMID: 34389764; PMCID: PMC8363750. 


\section{Abstract}

Chlamydia gallinacea is an obligate intracellular bacterium that has recently been added to the family of Chlamydiaceae. C. gallinacea is genetically diverse, widespread in poultry and a suspected cause of pneumonia in slaughterhouse workers. In poultry, C. gallinacea infections appear asymptomatic, but studies about the pathogenic potential are limited. In this study two novel sequence types of $C$. gallinacea were isolated from apparently healthy chickens. Both isolates (NL_G47 and NL_F725) were closely related to each other and have at least 99.5\% DNA sequence identity to C. gallinacea Type strain 08-1274/3. To gain further insight into the pathogenic potential, infection experiments in embryonated chicken eggs and comparative genomics with Chlamydia psittaci were performed. C. psittaci is a ubiquitous zoonotic pathogen of birds and mammals, and infection in poultry can result in severe systemic illness. In experiments with embryonated chicken eggs, C. gallinacea induced mortality was observed, potentially strain dependent, but lower compared to C. psittaci induced mortality. Comparative analyses confirmed all currently available $C$. gallinacea genomes possess the hallmark genes coding for known and potential virulence factors as found in C. psittaci albeit to a reduced number of orthologues or paralogs. The presence of potential virulence factors and the observed mortality in embryonated eggs indicates $C$. gallinacea should rather be considered as an opportunistic pathogen than an innocuous commensal. 


\section{Introduction}

Chlamydiaceae are a family of obligate intracellular bacteria containing one genus and 14 species, and comprising human and animal pathogens. In birds, infections are caused by Chlamydia psittaci or more recently recognized species such as C. gallinacea (1). C. psittaci is zoonotic and has been reported worldwide in more than 465 bird species belonging to at least 30 orders (2). Most human infections have been linked to contact with birds or their environments (3). C. gallinacea is mainly detected in poultry with reports from almost all continents (4-6). C. gallinacea has incidentally been found in wild birds and cattle as a possible result of infection spill-over $(7,8)$. Possible zoonotic transmission of $C$. gallinacea has been considered but could neither be confirmed nor ruled out in slaughterhouse workers that developed pneumonia after they were exposed to C. gallinacea infected poultry (9).

Infections with C. psittaci in birds are often asymptomatic, but can result in localized syndromes (e.g., conjunctivitis) or severe systemic illness. Chlamydial strain, avian host, host age and (environmental) stressors are important factors in the occurrence and severity of clinical signs (3). Studies investigating the pathogenesis of C. gallinacea in birds are currently limited. As yet, clinical signs of disease in C. gallinacea infections have not been reported in observational field studies $(4,9,10)$. Under experimental conditions it has been demonstrated that infection in broilers results in reduced weight gain (4). In a transmission study, C. gallinacea was mainly present in rectal and cloacal samples without clinical signs of disease and transmission occurred via the faecal-oral route (11). Thereby, at present $C$. gallinacea is considered a rather non-pathogenic species.

Molecular studies using outer membrane protein A (ompA) genotyping or Multi Locus Sequence Typing (MLST) showed C. gallinacea is diverse, with at least 13 different ompA types and 15 different sequence types (ST) in 25 strains $(4,12)$. Fine detail comparative genomics revealed that the $C$. gallinacea genome is conserved, syntenic and compact, but possesses the hallmark of chlamydial specific virulence factors: inclusion membrane (Inc) proteins, polymorphic membrane proteins (Pmps), a Type III Secretion System (T3SS), a plasticity zone with a cytotoxin (tox) gene, and the chlamydial virulence plasmid $(12,13)$. Whether this genetic diversity and the presence of chlamydial virulence genes contributes to the pathogenicity of $C$. gallinacea remains a question, as clinical disease in infected chickens has not been reported in the limited number of field and experimental studies.

The aim of this study was to investigate the pathogenicity of two novel C. gallinacea strains by comparing them to a virulent $C$. psittaci strain using an in vivo infection model in embryonated chicken eggs and performing comparative genomics with inter- and intra-species genomes. In the eggs, C. gallinacea induced mortality was observed, but to a lower extent than C. psittaci induced mortality. Comparative genomics showed that both 
novel C. gallinacea isolates possess the hallmark genes coding for known and potential virulence factors as found in C. psittaci, albeit to a reduced number of orthologs or alleles. The current results indicate C. gallinacea should be considered as an opportunistic pathogen rather than an innocuous commensal.

\section{Methods}

\section{Ethical statement and biosafety}

The cloacal and caecal sampling of the chickens was approved by the Dutch Central Authority for Scientific Procedures on Animals and the Animal Experiments Committee (permit number AVD108002016642) of Utrecht University (the Netherlands). All procedures were conducted in accordance with national regulations on animal experimentation and in compliance with the ARRIVE guidelines (14) where applicable. No ethical approval is required for work with embryonated chicken eggs until day 18 according to Dutch Law.

All culture work with C. gallinacea was performed under biosafety level 2 and all culture work with C. psittaci under biosafety level 3.

\section{Sample collection, inoculum preparation and isolation of Chlamydia}

Sample collection and inoculum preparation

Layer flocks at the Faculty of Veterinary Medicine in Utrecht, the Netherlands were monitored for the presence of $C$. gallinacea with boot sock sampling. The flocks were obtained from commercial laying hen rearing farms at 18 -weeks of age and had an average size of 50 hens that were distributed evenly over two pens. Background data on the flock are supplied in Supplementary Fig.. S1 and Supplementary Data S1. From each pen, environmental boot sock samples (Poultry Boot Swabs, BioTrading) were collected monthly. After collection, the boot socks were suspended in $100 \mathrm{ml}$ Dulbecco's Phosphate Buffered Saline (DPBS, Gibco, Life Technologies Limited). The suspension was centrifuged 15 minutes at $500 \times g$ and $500 \mu \mathrm{l}$ of the supernatant was used for DNA isolation. When the boot socks were PCR positive for Chlamydia, individual cloacal swabs and caeca were collected. Cloacal swabs were stored in one millilitre Sucrose Phosphate Glutamate (SPG) and caeca in ten percent weight per volume $(\mathrm{w} / \mathrm{v})$ according to standard protocols $(15,16)$. SPG contains sucrose $(75$ $\mathrm{g} /$ litre), $\mathrm{KH}_{2} \mathrm{PO}_{4}\left(0.52 \mathrm{~g} /\right.$ litre), $\mathrm{K}_{2} \mathrm{HPO}_{4}(1.25 \mathrm{~g} /$ /itre) and L-glutamic acid (0.92 g/litre). Before use, fetal bovine serum $(0.1 \mathrm{ml} / \mathrm{ml})$, amphotericin $B(4 \mu \mathrm{g} / \mathrm{ml})$, gentamicin $(40 \mu \mathrm{g} / \mathrm{ml}$ and vancomycin $(25 \mu \mathrm{g} / \mathrm{ml})$ were added. Samples were stored at $-80^{\circ} \mathrm{C}$.

To prepare the inoculum for the eggs, swabs were thawed at room temperature for approximately one hour. Swabs were centrifuged for ten minutes at $500 \times g$ and $200 \mu l$ of the supernatant was used for inoculation. Caeca were prepared following two methods. 
For the isolation of NL_G47 the caecum was cut lengthways in parts of approximately two $\mathrm{cm}$. Subsequently the parts were washed in SPG and the epithelium was removed by scraping with a scalpel. The scrapings of epithelium were washed in two $\mathrm{ml}$ of SPG and the suspension was filtered over a $0.8 \mu \mathrm{m}$ filter (Acrodisc Syringe Filter, Pall Life Sciences). After one $\mathrm{hr}$ of incubation at room temperature the suspension was used for inoculation. For the isolation of NL_F725, caeca were homogenized in a $10 \% \mathrm{w} / \mathrm{v}$ suspension in an ULTRATURRAX tube (BMT-20-S, IKA) on an ULTRA-TURRAX Tube Drive (IKA) at 6000 RPM for 90 seconds and switching direction every 30 s. The suspension was centrifuged at $500 \times g$ for $15 \mathrm{~min}$ and the supernatant was used for culturing as described below.

Inoculation of embryonated SPF chicken eggs

Specific-pathogen-free (SPF) embryonated chicken eggs were delivered after five days of incubation, candled to check viability and incubated overnight at $37.5-38{ }^{\circ} \mathrm{C}$ and $65 \%$ relative humidity in small egg incubators (Octagon 20 Advance, Brinsea). Inoculation was performed at day six of incubation (one day after delivery).

Before inoculation, the eggs were candled, and the air chamber was marked with a pencil. The eggs were cleaned with a wipe drenched in $70 \%$ ethanol. In the middle of the area of the marked air chamber, a hole was drilled with a $0.8 \mathrm{~mm}$ engraving bit (26150105JA, Dremel). Subsequently, the eggs were moved to a flow cabinet and sprayed with 70 percent ethanol. Per egg, $200 \mu \mathrm{l}$ was inoculated in the yolk sac with a one millilitre syringe and a $22 \mathrm{G} \times 40 \mathrm{~mm}$ needle. The full needle was inserted perpendicularly into the drilled hole.

Per clinical sample, four eggs were inoculated. As a negative control, two eggs were inoculated with DPBS (Gibco, Life Technologies Limited) and, as a positive control, two eggs were inoculated with C. gallinacea strain 08DC65. Strain 08DC65 was obtained from the Friedrich Loeffler Institute in Jena, Germany.

After inoculation eggs were wiped with $70 \%$ ethanol and the hole was closed with a droplet of nail polish. The eggs were placed in the egg incubators and incubated until day 16 or until mortality. At day 16 , eggs were chilled overnight at $4^{\circ} \mathrm{C}$ to euthanise the embryo non-invasively.

\section{Candling of embryonated SPF chicken eggs}

Mortality was monitored by daily candling. With candling, the appearance of vessels and movement of the embryo was monitored (17). The result of candling was graded:

- no abnormalities observed: vessels are visible, movement of the embryo

- abnormalities observed: congestion or bleeding from vessels, decreased movement of the embryo

- mortality: no or less vessels visible and no movement of the embryo 
When abnormalities were observed an extra candling was performed on the same day. After mortality or an increase in the severity of the abnormalities, eggs were chilled overnight at $4{ }^{\circ} \mathrm{C}$ until harvesting.

\section{Harvesting of embryonated SPF chicken eggs}

Mortality within three days after inoculation (day nine of incubation) was considered as acute mortality inconsistent with a Chlamydia infection (18). These eggs were disinfected with $70 \%$ ethanol, opened at the air sac side and checked for any visual deformations. Furthermore, a sheep blood agar plate was inoculated with a loopful from the yolk sac and incubated overnight at $37^{\circ} \mathrm{C}$ to check for bacterial contamination.

Eggs were harvested for the isolation of C. gallinacea when mortality occurred from day nine of incubation or when no mortality was observed at day 16 of incubation. At harvesting the part of the egg shell covering the air sac was removed, and subsequently the egg shell membrane and the allantois membrane were opened with disposable tweezers. The allantoic fluid was removed with a pipette, the egg was then emptied in a Petri dish to harvest the yolk sac membrane. The yolk sac membrane was weighed and transferred to an ULTRA-TURRAX tube (BMT-20-S, IKA). Depending on the volume of the yolk sac and the size of the tube, SPG buffer was added and the yolk sac membrane was homogenized on an ULTRA-TURRAX Tube Drive (IKA) for $90 \mathrm{~s}$ (switching between forward and reverse every $30 \mathrm{~s}$ ) at $6000 \mathrm{RPM}$. The suspension was transferred to $50 \mathrm{ml}$ Falcon tubes and SPG buffer was added until a $20 \% \mathrm{w} / \mathrm{v}$ suspension.

The yolk sac membranes from eggs inoculated with the same sample and harvested at the same day, were pooled to create one homogenous batch of an isolate. A $10 \mu \mathrm{l}$ droplet of the yolk sac suspension was spotted in duplo on glass slides and air dried. The glass slides were tested with the IMAGEN Chlamydia test kit (immunofluorescence test, IFT) according to manufacturer's instructions (Thermo Scientific). Two hundred $\mu$ l of the suspension was used for PCR testing.

\section{Isolation in cell culture}

Isolation and propagation in cell culture was performed as described earlier (19). Briefly, Buffalo Green Monkey (BGM) cells were seeded with Dulbecco's Modified Eagle Medium (DMEM, Gibco, Life Technologies Limited) and 10\% serum in 24-well plates (Greiner BioOne $\mathrm{GmbH}$, Germany). The plates were incubated at $37{ }^{\circ} \mathrm{C}$ with $5 \% \mathrm{CO}_{2}$ in a humidified incubator until $80 \%$ confluency of the monolayer. After inoculation, the plates were centrifuged at $2450 \times g$ and $37^{\circ} \mathrm{C}$ for $60 \mathrm{~min}$ and subsequently incubated for two hours. The medium was then replaced with UltraMDCK serum-free medium (Lonza). At day one and day four, $200 \mu \mathrm{l}$ of the supernatant was collected for PCR to monitor replication. Plates were harvested at day four for DNA isolation, further passaging or storage at $-80^{\circ} \mathrm{C}$. 


\section{Titration experiments in embryonated SPF chicken eggs}

The isolated C. gallinacea strains NL_G47 and NL_F725, and C. psittaci strain NL_Borg were tested in titration experiments. Strain NL_Borg was selected because it is genetically closely related to strain FalTex and NJ1, which are both isolated from outbreaks in poultry (turkeys) (20).

To standardise the inocula before the start of the titration experiments, all three strains were passaged three times in embryonated eggs under similar conditions. The third passage yolk sac membrane suspensions were used to prepare tenfold serial dilutions in DPBS (Gibco, Life Technologies Limited) for inoculation of the yolk sac of six-day incubated chicken eggs. The eggs were incubated at $37{ }^{\circ} \mathrm{C}$ and $65 \%$ relative humidity in egg incubators (Octagon 20 Advance, Brinsea). After mortality or six days after inoculation the eggs were chilled overnight at $4{ }^{\circ} \mathrm{C}$ and harvested as described earlier.

In a first experiment the range for the dilution series was defined by inoculating a limited number of eggs per dilution. In a subsequent experiment the range was limited to four dilution steps. Per dilution step, four or five eggs were inoculated with $200 \mu$ l suspension. Two or more eggs were inoculated with sterile DPBS (Gibco, Life Technologies Limited) as a negative control and, as a positive control, two eggs were inoculated with a lower dilution than the range that was used in the experiment.

After each titration experiment the $50 \%$ egg infective dose $\left(E_{1 D_{50}}\right)$ and, when possible, the $50 \%$ egg lethal dose $\left(\mathrm{LD}_{50}\right)$ per $\mathrm{ml}$ inoculum was calculated according the SpearmanKarber method $(21,22)$. The difference in EID $_{50}$ between strains was assessed using the Wilcoxon-Mann-Whitney test.

\section{Histology and immunohistochemistry}

From infected and non-infected eggs, the chorioallantoic membrane, yolk sac and embryo were harvested for histology and immunohistochemistry. After fixation in 10\% neutral buffered formalin, tissues were routinely processed into paraffin blocks. Four $\mu \mathrm{m}$ sections were cut and collected on coated glass slides. Sections were stained with haematoxylineosin (HE) or immuno-stained with a polyclonal anti-Chlamydia antibody (LS-C85741) and a monoclonal anti-Chlamydia antibody (MBS830551).

For the polyclonal antibody the antigen was retrieved by proteolysis-induced epitope retrieval $\left(0.1 \%\right.$ Trypsin in TBS for $30 \mathrm{~min}$ at $\left.37^{\circ} \mathrm{C}\right)$. For the monoclonal antibody heatinduced epitope retrieval was used (citrate buffer, $\mathrm{pH} 6.0,21^{\circ} \mathrm{C}$ for five $\mathrm{min}$ ). The primary antibody (dilution 1:100) was incubated for $60 \mathrm{~min}$. HRP EnVision anti-Mouse or HRP Envision anti-Rabbit (Dakopatts) were used as a secondary antibody for $30 \mathrm{~min}$, depending 
on the nature of the first antibody. Subsequently, sections were incubated for five min in $\mathrm{DAB}+$ substrate (Dakopatts) and then counterstained with Mayer's haematoxylin.

\section{DNA extraction, PCR and genome sequencing}

Five hundred $\mu \mathrm{l}$ of the sample suspensions, washing suspension, yolk sac suspension or cell culture supernatant was used for DNA extraction. DNA extraction was performed with a MagNA Pure LC total Nucleic Acid Isolation kit in the MagNA Pure system (Roche Diagnostics, Almere, the Netherlands). Samples were tested with a Chlamydiaceae PCR targeting the $23 \mathrm{~S}$ rRNA and $C$. gallinacea PCR targeting the enoA gene or C. psittaci PCR targeting the ompA gene as described earlier $(10,23)$.

For genome sequencing, twenty-four-well cell culture plates were freeze-thawed twice and the cells were subsequently harvested for DNA extraction as described earlier (19). DNA was isolated according to the DNeasy Blood and Tissue kit (Qiagen GmbH, Germany).

The DNA samples were prepared for Illumina sequencing using the SMARTer ThruPLEX DNA-Seq kit (Takara Bio, USA) according to manufacturer protocol. Quality control of the library preparation was performed on a Tapestation 2200 (Agilent Technologies, Germany) and the DNA concentration was determined on a Clariostar (BMG Labtech, the Netherlands) with use of the Quant-IT PicoGreen dsDNA kit (Invitrogen Ltd, UK). Sequencing was performed on an Illumina MiSeq platform. The complete genome and plasmid sequences were assembled using SKESA 2.4.0 (24). Contigs containing sequences of BGM cells were removed prior to subsequent analysis.

Assembled contigs (from Illumina short reads) were annotated using the PGAP pipeline using C. gallinacea Type Strain 08-1274/3 (accession number NZ_CP015840.1) as the reference genome for the newly isolated C. gallinacea strains and C. psittaci NJ1 (accession number CP003798.1) for C. psittaci NL_Borg (25). All data are available in the $\mathrm{NCBI}$ database under BioProject number PRJNA687129 (including reads available under SRR15184193; SRR15184194 and SRR15212117) and the publicly available Bacterial Isolate Genome Sequence Database (BIGSdb) ((http://pubmlst.org/chlamydiales) (C. gallinacea isolates NL_G47 (id: 4548) and NL_725 (id: 4560) and C. psittaci NL_Borg (id: 4561)).

\section{Molecular typing and phylogenetic analysis}

Sequence types for our strains were determined using contigs deposited and queried against the Chlamydiales PubMLST database (http://pubmlst.org/chlamydiales). Phylogenetic trees were generated by exporting gene sequences from the Chlamydiales database (http://pubmlst.org/chlamydiales) as an XMFA file containing each locus as an aligned block. The XMFA file was converted to an aligned concatenated sequence 
for Neighbor-Joining tree analysis using the Maximum Composite Likelihood model in MEGA7(26). Bootstrap tests were for 1000 repetitions (27-29).

For rMLST, complete sequences ( 22.000 bp) of 52 genes encoding ribosomal proteins (rps) were analysed (30). The rps gene $r p m D$, encoding the $50 \mathrm{~S}$ ribosomal protein $\mathrm{L} 30$ is absent in genomes of Chlamydia isolates analysed so far. For MLST, sequences of fragments (400 - 500 base pairs) from seven housekeeping genes (enoA, fumC, gatA, gidA, hemN, hIfX, oppA) were analysed (31). Isolates used for rMLST and MLST including provenance and allelic profile data are listed in Supplementary Data $\mathrm{S6.}$

\section{Comparative genome analyses}

Average nucleotide identity (ANI) determination for the newly sequenced $C$. gallinacea genomes was performed using the ANI calculator available at enve-omics.ce.gatech.edu/ ani/ (32), whilst the genome completeness based on the percent of bases aligned to the reference genome and quality of the assemblies was estimated using Quast (32-34). SNPs in contigs assembled from Illumina reads, were identified using Snippy v4.6.0(35).

C. gallinacea pairwise genome comparisons were performed using the Geneious Prime 2020.2 platform (https://www.geneious.com). Our strains were compared against C. gallinacea strain 08-1274/3 (accession number NZ_CP015840.1) and JX-1 (accession number CP019792). The genomic regions of interest and/or polymorphic loci were extracted from the analysed genomes and aligned with MAFFT and/or Clustal Omega (as implemented in Geneious Prime) for further nucleotide and/or translated protein sequence analyses performed using DNASp 6.0 (36). The total number of polymorphisms (and gaps), \% nucleotide and amino acid sequence identity, number of haplotypes and haplotype diversity $(\mathrm{Hd})$, and ratios of the rates of non-synonymous to synonymous nucleotide substitutions per site (dn/ds) averaged over the entire gene alignment were calculated.

As the Type 3 Secretion System (T3SS) play a key role in the interaction of Chlamydia and hosts, EffectiveDB (http://effectivedb.org) was used to predict the T3S secreted proteins of $C$. gallinacea. For prediction the standard Effective T3 classification module 2.0.1 was used with a cut-off score of 0.9999 (37). Similarly, to predict transmembrane $C$. gallinacea proteins, and identify inclusion membrane proteins characterised by bilobed hydrophobic domains, TMHMM 2.0 server (https://services.healthtech.dtu.dk/service. php?TMHMM-2.0) was used (38).

The visualisation of nucleotide BLAST comparisons of our newly sequenced draft $C$. gallinacea genomes to published C. gallinacea genomes 08-1274/3 and JX-1, and/or $C$. psittaci NJ1 (accession number CP003798.1) was performed with BLAST Ring Image 
Generator (BRIG)(39). Visualisation of the BLAST comparison, sequence identity and genomic structure of the plasticity zone for C. gallinacea and those from other related species, was performed using EasyFig, with the -tblastx option with a minimum E-value of $1 \times 10^{-3}$ used as BLAST parameters for EasyFig (40).

For the identification of orthologous genes in C. gallinacea and C. psittaci, an all-vsall comparison of the translated coding sequences (CDSs) was performed using global sequence alignment of each CDS. Translated CDSs were aligned using DIAMOND v0.9.14 and the best hit for each query was selected (41). Only hits with an expect (E) value less than $1 \times 10^{-3}$ were included. CDS with no hits or hits with an E-value above the threshold were further investigated and the annotation artefacts were removed. The remaining CDS were assigned unique. In addition, all CDS were investigated using both nucleotide and translated amino acid sequence blast analyses. Results of the alignment were structured and visualized using the tidyverse package and $\mathrm{R}$ v3.6.1 $(42,43)$.

\section{Results}

\section{Isolation and pathology of C.gallinacea NL_G47 and NL_F725 in embryonated chicken eggs}

Layer flocks at the Faculty of Veterinary Medicine in Utrecht, the Netherlands were monitored for the presence of $C$. gallinacea to isolate Dutch field strains. In these flocks, C. gallinacea strain NL_G47 could be isolated from a caecal scraping sample collected in January 2018 from a 40-week old clinically healthy layer hen. C. gallinacea strain NL_ F725 could be isolated from a caecal suspension sample collected in August 2017 from a 34-week old layer hen. Both hens originated from different flocks, but were housed at the same location at different time points. About one month before the $C$. gallinacea positive caecal samples were collected, both flocks tested PCR positive for $C$. gallinacea in environmental boot sock samples as shown in the timeline of Supplementary Fig. S1. C. gallinacea positivity in the flock from strain NL_F725 preceded a coinciding Infectious Laryngotracheitis (ILT) infection. To prevent further spread of ILT the flock had to be culled. Background data of the flocks are added to Supplementary Data S1.

C. gallinacea NL_G47 and NL_F725 were isolated in the yolk sac of embryonated specific-pathogen-free (SPF) chicken eggs and replication was confirmed with positive immunofluorescence of the yolk sac membrane (see Supplementary Fig. S2) and a positive Chlamydiaceae PCR targeting the 23S rRNA gene. With the isolation of NL_G47 in the yolk sac of embryonated eggs, mortality was observed at day 10 after inoculation (incubation day 16) and at day 6 (incubation day 12) in the second passage. At primary isolation of NL_F725 no mortality of the embryos was observed, but eggs were harvested before day 
10 after inoculation (day 8 after inoculation, incubation day 14) for logistical reasons. With the second passage of NL_F725, mortality of the embryos was observed at day 6 or day 7 after inoculation (incubation day 12 or 13). Based on egg candling, congestion of the blood vessels was observed prior to mortality of the embryos. At harvest the embryos were deep red (rubor), showed cyanotic toes and haemorrhaging of the skin (Supplementary Fig. S2).

To investigate any histological lesions NL_G47 infected eggs were harvested at day 10 of incubation when anomalies of the vessels were observed with candling. Granular basophilic intracellular inclusions were seen in the epithelial cells of both the chorioallantoic membrane and the yolk sac membrane (Figs $1 \mathrm{~A}$ and $\mathrm{C}$ ). These intracellular inclusions were strongly positive for chlamydial antigen labelling (Fig. 1B, D).
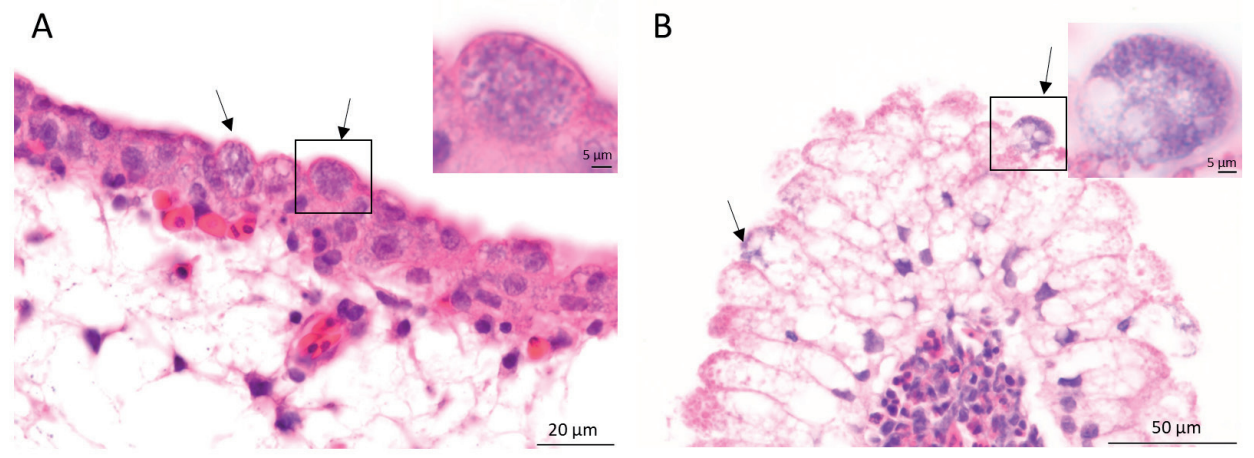

C

D
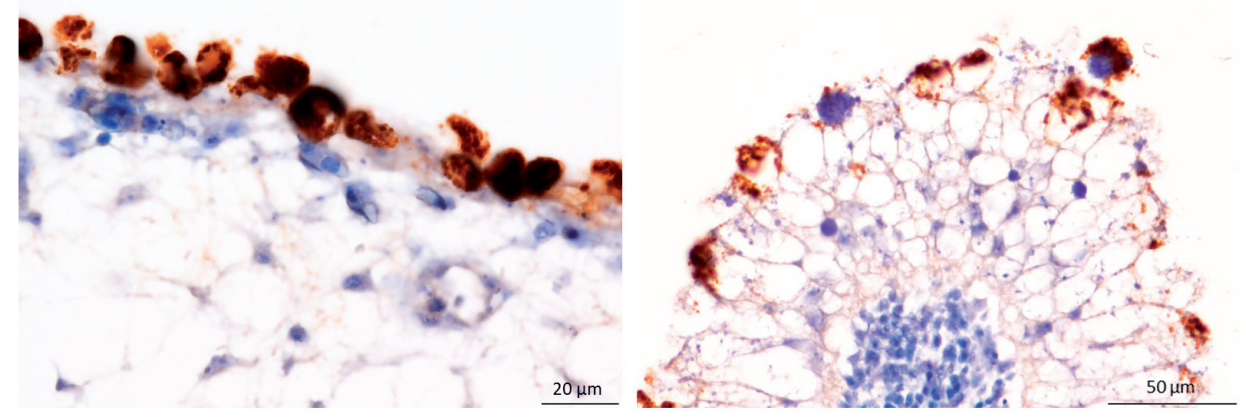

Fig. 1. Chorioallantoic membrane and yolk sac membrane of 10 days embryonated eggs infected with NL_G47

Intracellular inclusions (arrows) in the epithelial cells of the chorioallantoic membrane (A) and yolk sac membrane (B). Inset: higher magnification showing the granular basophilic inclusions in the HE staining. Positive immunolabelling of the intracellular inclusions for chlamydial antigen in the chorioallantoic membrane (C) and yolk sac membrane (D). Sections were photographed with an Olympus BX51 microscope equipped with a high-resolution digital camera and using Olympus' cellSens software. 
Primary isolation and propagation of C. gallinacea NL_G47 and NL_F725 in Buffalo Green Monkey (BGM) cells initially failed, but after three passages in eggs the strains could be propagated in BGM cells.

\section{Assessment of virulence of $C$. gallinacea in embryonated eggs}

Titration experiments in embryonated chicken eggs were performed to quantify the infectious dose and gain further insight into the pathogenic potential of the novel isolates compared to $C$. psittaci. Ten-fold serial dilutions of third passage yolk sac cultures of $C$. gallinacea NL_G47 and NL_F725, and C. psittaci NL_Borg, were used to calculate the 50\% egg infective dose (EID ${ }_{50}$ ) based on positivity in the immunofluorescence test (IFT) of the yolk sac membrane (with or without mortality of the eggs). The experiments were repeated seven times for NL_G47 with a median EID 50 of $10^{5.6}$, two times for NL_F725 with a median EID $_{50}$ of $10^{5.9}$ and three times for NL_Borg with a median EID F0 $^{8.2}$. All negative control eggs that were inoculated with Dulbecco's Phosphate Buffered Saline (DPBS), remained viable until harvesting and tested Chlamydia negative by the IFT, except in one experiment with NL_G47 where aspecific mortality was observed in two of four eggs within three days after inoculation. As shown in Fig. $2 A$, the $E_{50}$ of $C$. psittaci strain $\mathrm{NL}$ BBorg was significantly higher than the $\operatorname{EID}_{50}(\mathrm{P}<0.05$, Wilcoxon-Mann-Whitney test) of C. gallinacea NL_G47. The EID 50 of NL_F725 was in the same range as the EID 50 of NL_G47, but could not be statistically assessed due the low number of observations.

For C. psittaci NL_Borg the $50 \%$ lethal dose $\left(\mathrm{LD}_{50}\right)$ could also be calculated from the experiments with a median $\mathrm{LD}_{50}$ of $10^{7.4}$. The $\mathrm{LD}_{50}$ of the experiments with $C$. psittaci NLBorg showed overlap with the calculated EID $_{50}$ (Fig 2A). The $L_{50}$ from the experiments with C. gallinacea NL_G47 and NL_F725 could not be calculated, because the number of eggs in the dilutions with observed mortality was too low to calculate the $\mathrm{LD}_{50}$. To get further insight into differences in mortality and infectivity between C. gallinacea and C. psittaci, the data from all separate experiments were merged into one dataset (see Supplementary Table S1).

The percentage of eggs that was IFT positive with mortality, IFT positive without mortality and IFT negative is shown per dilution and per Chlamydia strain (Figs 2B-D). For C. gallinacea strain NL_G47, mortality was observed until the $10^{-2}$ dilution and IFT positivity until the $10^{-6}$ dilution (Fig. 2B). For C. gallinacea strain NL_F725 no mortality was observed in the dilutions that were tested (from $10^{-2}$ until $10^{-7}$ ), but IFT positivity was seen until the $10^{-6}$ dilution similar to C. gallinacea NL_G47 (Fig. 2C). For C. psittaci strain NL_Borg, mortality was observed until dilution $10^{-7}$ and IFT positivity until $10^{-8}$ (Fig. 2D). These results indicate mortality in the $C$. psittaci infected eggs was relatively higher than in the $C$. gallinacea infected eggs and there might be a difference in mortality between C. gallinacea strains, although the number of observations was low. 
A

Results per titration experiment

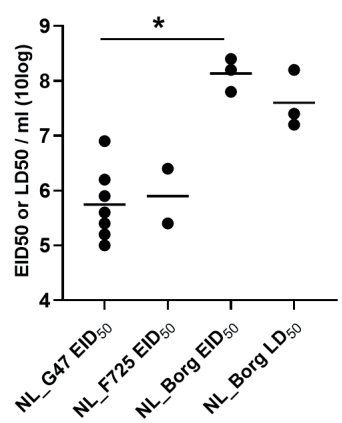

C

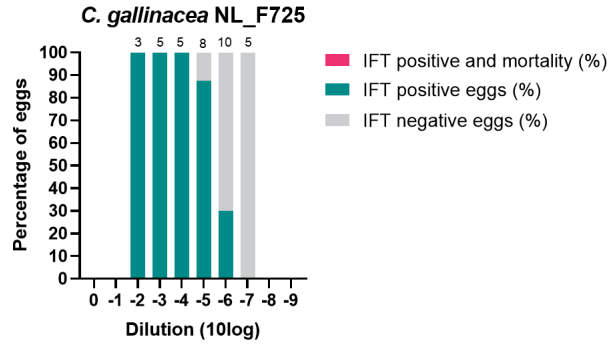

B

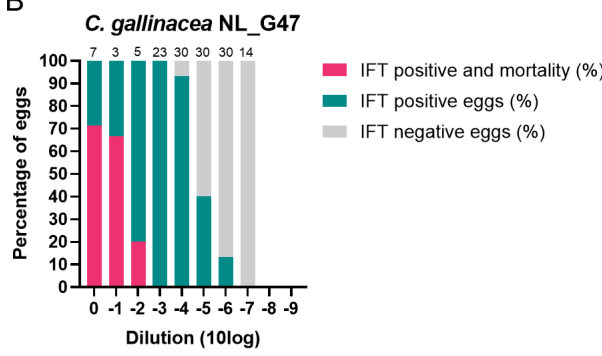

D

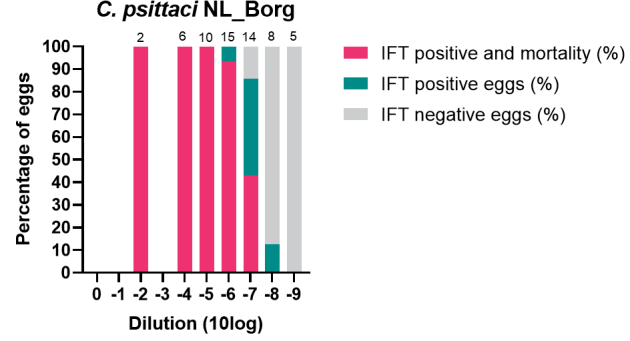

Fig. 2. Assessment of virulence of $C$. gallinacea in embryonated eggs

A shows the $50 \%$ egg infective dose 50 (EID 50 ) of C. gallinacea NL_G47, NL_F725 and C. psittaci NL_Borg based on IFT of the yolk sac. The difference between EID ${ }_{50}$ of NL_G47 and NL_Borg was significantly different $\left({ }^{*}, P<0.05\right.$, Wilcoxon-Mann-Whitney test). For $C$. psittaci NL_Borg the $50 \%$ lethal dose $\left(L D_{50}\right)$ was also calculated. The median EID ${ }_{50}$ or $L D_{50}$ of the experiments is indicated with a bar.

B, $\mathbf{C}$ and $\mathbf{D}$ depict the cumulative results of the separate titration experiments per Chlamydia strain. Per dilution, the percentage of eggs that was positive for Chlamydia in the immunofluorescence test (IFT) with mortality, IFT positive without mortality and IFT negative are shown. The total number of eggs per dilution are presented at the top of every bar. These data are also included in Supplementary Table S1. The figure was created in GraphPad Prism 9.0.0.

\section{General characteristics of the genome sequences of Dutch C. gallinacea isolates}

After isolation in eggs and one passage in BGM cells, DNA of both isolates was sequenced to confirm their genetic identity. The genomes of NL_G47 and NL_F725 have a total length of 1,066,007 and 1,064,097 bp, respectively, and include the $\sim 1.059 \mathrm{Mbp}$ chromosome and a 7.5 kbp chlamydial plasmid (Table 1). Ribosomal MLST (rMLST) (30) and phylogenetic analysis of concatenated rRNA genes confirmed that both isolates belong to C. gallinacea (Fig. 3A), whilst the MLST showed that both isolates are genetically diverse and assigned to unique sequence types (ST280 and ST284). Phylogenetically, these clustered in distinct clades, with NL_G47 forming a well-supported clade with the French isolate 08-1274/3, whilst NL_F725 clustered in a genetically diverse clade consisting of Chinese C. gallinacea strains (Fig. 3B). 
Table 1.Genome descriptions of C. gallinacea NL_G47, C. gallinacea NL_F725 and C. psittaci NL_Borg

\begin{tabular}{|c|c|c|c|}
\hline & C. gallinacea NL_G47 & C. gallinacea NL_F725 & C. psittaci NL_Borg \\
\hline Host & Chicken (Gallus gallus) & Chicken (Gallus gallus) & $\begin{array}{c}\text { In-house reference } \\
\text { strain }\end{array}$ \\
\hline Anatomical site & Caecum & Caecum & Unknown \\
\hline Clinical presentation & Asymptomatic & Asymptomatic & Unknown \\
\hline Total No. of Illumina reads & 1912918 & 1762101 & 3029302 \\
\hline Percent of mapped reads & $72.92 \%$ & $73.06 \%$ & $93.46 \%$ \\
\hline No. of de novo contigs ${ }^{a}$ & 12 & 19 & 11 \\
\hline N50 & 114660 & 96259 & 254182 \\
\hline Average coverage depth & $366 X$ & $181 X$ & $633 X$ \\
\hline$\% G C$ of de novo contigs & $37.89 \%$ & $37.89 \%$ & $38.92 \%$ \\
\hline $\begin{array}{l}\text { Number of bp mapped against } \\
\text { reference genome chromosome } \\
\text { (\% complete compared to } \\
\text { reference strains }{ }^{b} \text { ) }\end{array}$ & 1058515 bp (99.89\%) & 1057023 bp (99.75\%) & 1144332 bp (98.5\%) \\
\hline $\begin{array}{l}\text { Number of bp mapped against } \\
\text { the reference plasmid }\end{array}$ & 7492 bp & 7492 bp & 7552 bp \\
\hline Number of predicted CDS & 916 & 919 & 989 \\
\hline \multirow{3}{*}{$\%$ Average nucleotide identityc } & $\begin{array}{c}\text { 99.63\% (SD: } 1.09 \%) \\
\text { to C. gallinacea } \\
\text { 08_1274/3 }\end{array}$ & $\begin{array}{c}\text { 99.50\% (SD: } 1.53 \% \\
\text { to C. gallinacea } \\
08 \_1274 / 3\end{array}$ & - \\
\hline & $\begin{array}{l}99.42 \% \text { (SD: } 1.43 \%) \\
\text { to C. gallinacea JX-1 }\end{array}$ & $\begin{array}{l}99.52 \% \text { (SD: } 1.31 \%) \\
\text { to C. gallinacea JX-1 }\end{array}$ & - \\
\hline & - & - & $\begin{array}{c}99.99 \% \text { (SD: } 0.04 \%) \\
\text { to C.psittaci NJ1 }\end{array}$ \\
\hline No of SNPs to reference strains ${ }^{d}$ & $\begin{array}{c}2608 \\
\text { to C. gallinacea } \\
08 \_1274 / 3 \\
\end{array}$ & $\begin{array}{c}3328 \\
\text { to C. gallinacea } \\
08 \_1274 / 3 \\
\end{array}$ & $\begin{array}{c}65 \\
\text { to C. psittaci NJ1 }\end{array}$ \\
\hline Plasticity Zone length & $15861 b p$ & $15845 \mathrm{bp}$ & 29000bp \\
\hline Accession numbers & JAEMHG000000000 & JAEMHН000000000 & - \\
\hline
\end{tabular}

a de novo chlamydial contigs; $b$ Quast analyses using Short read assemblies where NL_G47 and NL_F725 were compared to 08_1274/3, and NL_Borg with NJ1; C Average nucleotide identity (ANI) determination was performed at enve-omics.ce.gatech.edu/ani/ (Goris et al., 2007) using both best hits (one-way ANI) and reciprocal best hits (two-way ANI) between two genomic datasets with C. gallinacea 08_1274/3, C. gallinaceae JX-1 or C. psittaci NJ1 as the reference genome; $d$ SNPs identified using Snippy v4.6.0. 
A

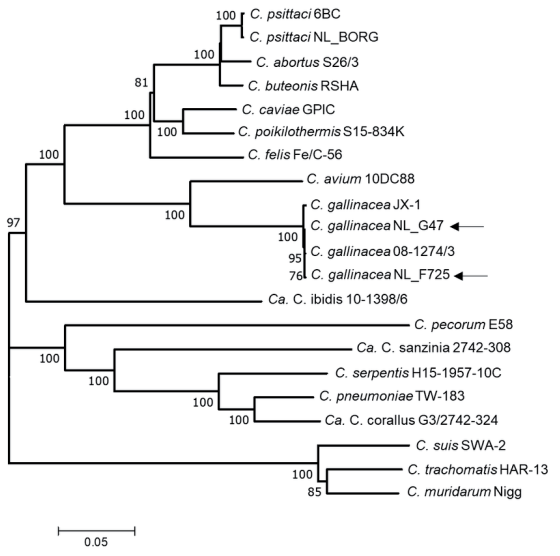

B

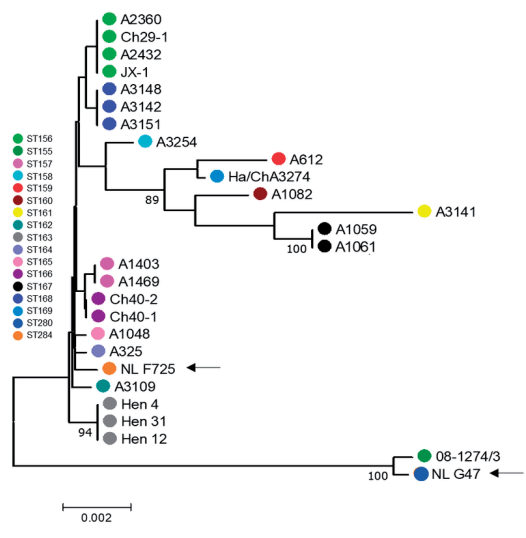

Fig. 3. Phylogenetic analyses of concatenated sequences of Chlamydia.

Concatenated sequences were aligned and analysed in MEGA7(26).

Numbers on tree nodes indicate bootstrap values over $75 \%$ of the main branches. Horizontal lines are scale for nucleotide substitutions per site.

A Neighbor-Joining tree of concatenated sequences of 52 ribosomal genes (rMLST)(30) of Chlamydia Type strains as well as three Candidatus species (Ca. C. corallus, Ca. C. ibidis and Ca. C. sanzinia), C. psittaci strain NL_Borg and two additional C. gallinacea strains. All C. gallinacea strains (Dutch strains indicated by an arrow) clustered together in a well-supported and distinct clade with Chlamydia avium as the closest relative.

B Neighbor-Joining tree of concatenated sequences of 7 housekeeping genes fragments (MLST)(31) of 27 C. gallinacea strains. Shared Sequence types (ST) in clades are indicated by color and STs are denoted by the color key.

\section{Comparative genome analysis of $C$. gallinacea and C. psittaci}

To investigate genomic differences that might be related to the observed differences in the degree of pathology and mortality in eggs, the C. gallinacea and C. psittaci genomes were analysed and compared. As evaluated by whole genome alignments, $C$. gallinacea genomes NL_G47 and NL_F725 are syntenic with the same gene number and order, sharing at least $99.4 \%$ sequence identity with C. gallinacea strain 08-1274/3 (type strain; accession number NZ_CP015840.1) and JX-1 (accession number CP019792). All C. gallinacea genomes contain conserved hallmark chlamydial virulence genes coding for Incs, Pmps, T3SS and a Plasticity Zone (PZ) with a gene coding for the large cytotoxin (toxB) (Fig. 4A, C, Supplementary Fig. S3). Most sequence variation was found in several distinct chromosomal regions, namely in genes encoding the membrane proteins (e.g. ompA and pmps), a conserved hypothetical protein, a phage tail protein, heme (hemE, and hemN) and glycogen ( $g / g P$ ) metabolism genes (Supplementary Data S2). The PZ, a region of high genetic variability in chlamydial species, was conserved in number of genes and sequence among the four C. gallinacea genomes with $99.3-99.8 \%$ nucleotide identity, but varied in 
gene content, namely lack of hypothetical protein, MAC/Perforin (MAC/P) and nucleotide metabolism genes, compared to the related avian species (Fig. 4C). Although, the length of the PZ of $C$. gallinacea is reduced compared to $C$. psittaci, it does contain an intact CDS for the cytotoxin (toxB), in contrast to the PZ of $C$. avium that lacks this gene. As observed previously, this locus has a premature stop codon in strain JX-1 (Fig. 4C).

The genome sequence of our in-house reference strain C. psittaci NL_Borg was almost identical (99.99\% sequence identity) to reference strain C. psittaci NJ1 (accession number CP003798.1) with only 65 synonymous Single Nucleotide Polymorphisms (SNPs), evenly distributed across the chromosome. In the whole genome alignment, it was observed that the C. psittaci genome is $101.85 \mathrm{Kbp}$ longer than the genome of $C$. gallinacea and contains 73 more CDSs (Fig. 4A).

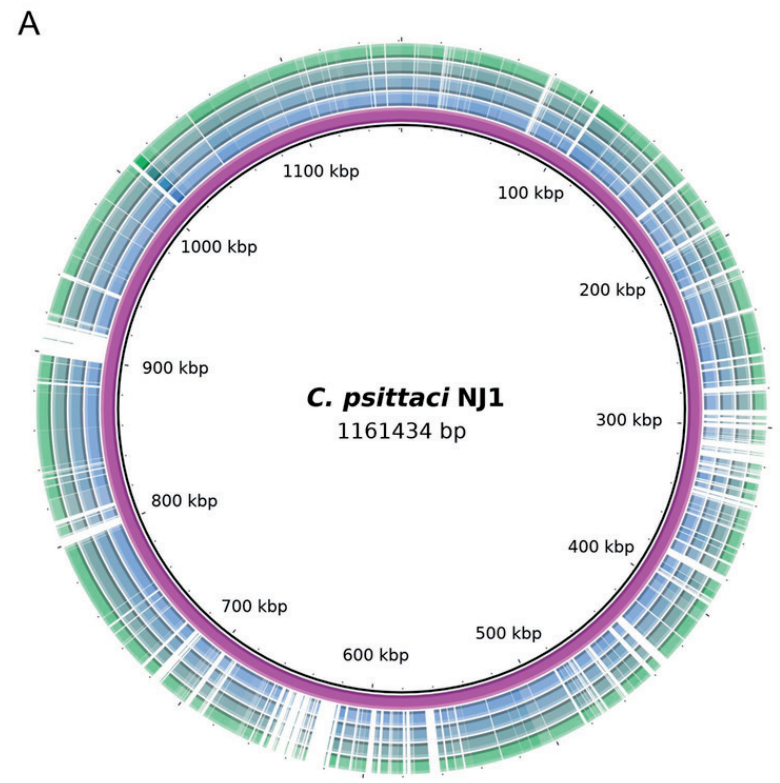

\author{
C. psittaci NJ1 \\ $100 \%$ identity \\ $70 \%$ identity \\ $50 \%$ identity \\ C. gallinacea 08-1274 \\ $100 \%$ identity \\ $70 \%$ identity \\ $50 \%$ identity \\ C. gallinacea JX-1 \\ $100 \%$ identity \\ $70 \%$ identity \\ $50 \%$ identity \\ C. gallinacea NL G47 \\ $100 \%$ identity \\ $70 \%$ identity \\ $50 \%$ identity \\ C. gallinacea NL F725 \\ $100 \%$ identity \\ $70 \%$ identity \\ $50 \%$ identity
}




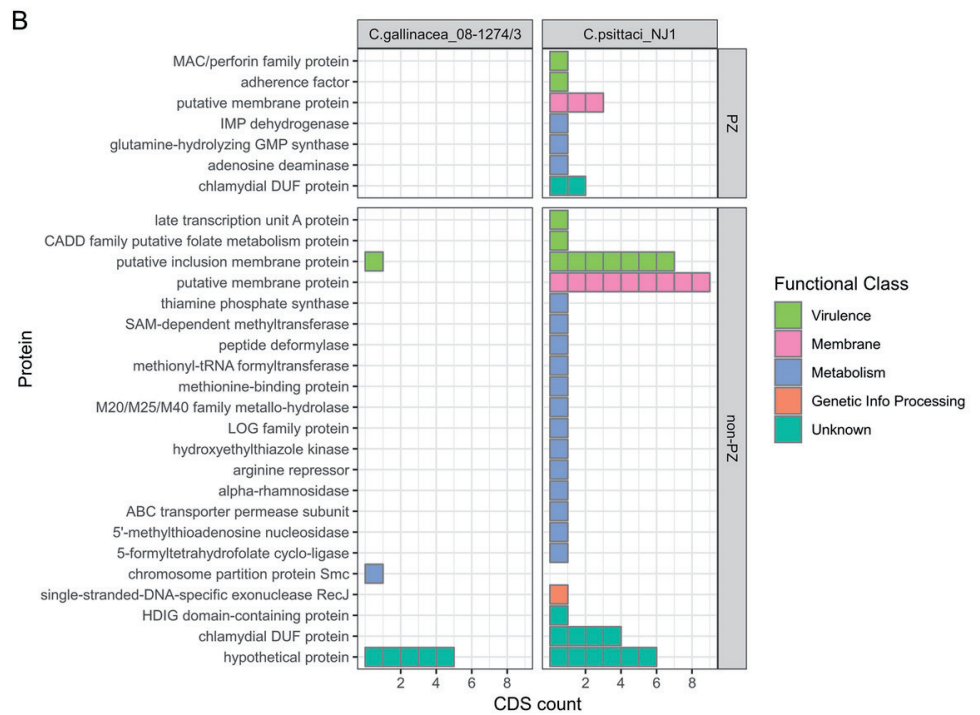

C. ibidis $10-1398 / 6$ C. abortus 15-70d24 C. buteonis RSHA C. psittaci N 1

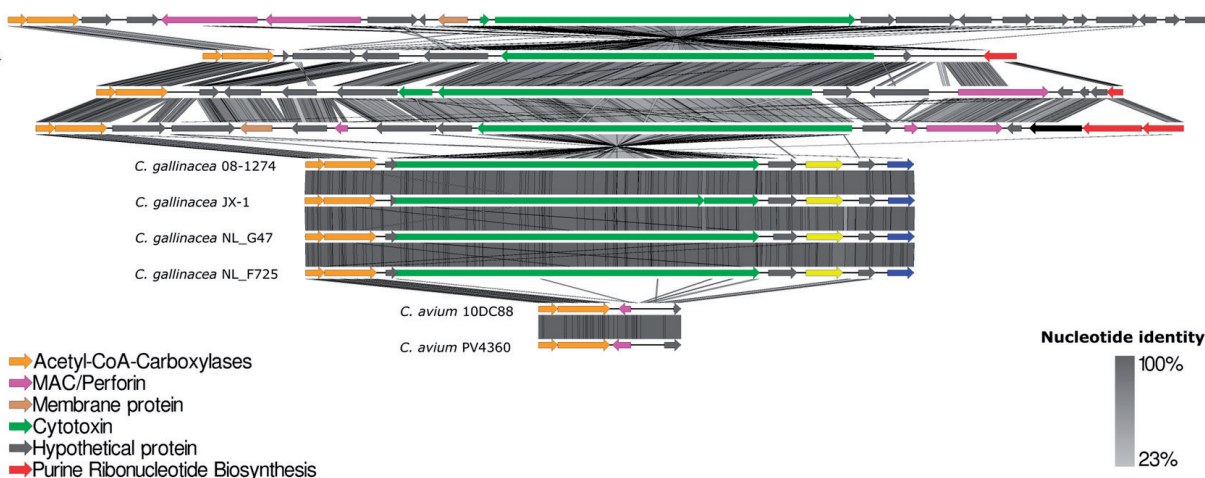
$\Rightarrow$ Hypothetical protein

$\Rightarrow$ Purine Ribonucleotide Biosynthesis

$\Rightarrow$ Adenosine/AMP deaminases

Gamma-glutamylcyclotransferase

$\Rightarrow$ Queuosine precursor transporter

Fig. 4. Genome comparison of C. gallinacea and C. psittaci.

A Whole genome BLAST comparison between C. psittaci NJ1 and four C. gallinacea genomes (including Type strain 08-1274/03). The image is created with BLAST Ring Image Generator (BRIG)(39) and the first ring corresponds to the genome that was used for the comparison.

BCDS for which no homologue (alignment $E$ score higher than $1 \times 10^{-3}$ ) could be identified in C. gallinacea 08-1274/03 or C. psittaci NJ1. Every colored block in the figure corresponds to a CDS. The different proteins are categorized and colored according to their function and location. The figure was created using the tidyverse package and $R$ v3.6.1(42, 43)

$C$ Graphical representation of the gene content of the PZs of representative Chlamydia species of avian origin including the Dutch C. gallinacea strains. Arrows represent $P Z$ genes colored according to function (see key). Grey shading scale denotes \% nucleotide identity. The image was created with Easyfig(40) . 
Given that our newly sequenced genomes are syntenic and almost identical to the comparator reference genomes, but only cover $98.51-99.75 \%$ of the reference chromosome lengths (Table 1), genomes of the type strains 08-1274/3 and NJ1 were used as representatives for $C$. gallinacea and C. psittaci species, respectively, in a translated coding sequences (CDSs) comparison. With a local alignment approach, all translated CDSs of C. gallinacea 08-1274/3 $(n=913)$ and C. psittaci NJ1 $(n=986)$ were compared to each other to identify unique and/or highly variable regions (Supplementary Data S3). The plasmids of C. gallinacea and C. psittaci were not included, because they are syntenic with both eight CDSs encoding the conserved chlamydial plasmid proteins.

As expected in closely related species and analysed by both amino acid and sequence similarity analyses, the majority of CDSs have orthologues in both species. In C. gallinacea, for only seven CDSs an orthologue could not be identified in C. psittaci (Fig. 4B, Supplementary Data S4). Of those, one belonged to the family of putative Incs, a second had a metabolic function related to chromosome partition and the remaining five were hypothetical proteins with unknown function. Fifty-three CDS were unique to C. psittaci relative to C. gallinacea (Supplementary Data S4). Ten of these CDSs were located at the PZ coding for proteins such as the Membrane Attack Complex/Perforin domain-containing protein (MAC/PF), proteins involved in purine metabolism (guaAB-ADA operon), adherence domain and a putative membrane protein.

Outside the $P Z, 18$ of the unique CDS of $C$. psittaci were related to previously characterised potential virulence factors (Fig. 4B, Supplementary Data S4). Most of these proteins belonged to the family of putative Inc proteins, membrane proteins and conserved hypothetical proteins. The remaining unique CDS were related to metabolism or to CDS coding for proteins of unknown function. Additional analysis of secretion signals of T3SS effector CDSs, important in Chlamydia virulence, revealed that a serine protease referred to as chlamydial protease-like activating factor (CPAF) is not predicted to be secreted in C. gallinacea in contrast to C. psittaci (44). However, C. psittaci orthologues of the recently described T3SS that associate with the host's inner nuclear membrane (SINC), and translocated actin-recruiting phosphoprotein (TARP) were identified and predicted to be secreted (Supplementary Data S5).

Overall, the analysis revealed the novel C. gallinacea genomes NL_G47 and NL_F725 have at least $99.5 \%$ sequence identity to the Type strain 08-1274/3 and include the hallmark chlamydial virulence genes. However, C. psittaci has a larger set of genes that are related to virulence and metabolism, including more incs, pmps, T3SS effectors and additional genes in the $\mathrm{PZ}$. 


\section{Discussion}

In this study, the pathogenic potential of two new chicken-derived C. gallinacea strains (NL_G47 and NL_F725) were investigated combining classical in vitro methods using embryonated chicken eggs and whole-genome analyses. During isolation of NL_G47 and NL_F725, pathogenic changes were observed that also have been described for other Chlamydia species (18), such as deep red colour (rubor), cyanotic toes and skin haemorrhage of the embryo. Mortality in embryonated eggs after yolk sac inoculation with C. gallinacea has been reported by Guo et al.(4), but was not mentioned by Laroucau et al. (9).

The layer flocks from which the strains originated were apparently healthy, which is in line with observations from other field studies $(4,9,10)$. It could not be evaluated if $C$. gallinacea infection led to impaired production as data on egg production were not collected in this teaching flock. The duration and frequency of shedding during C. gallinacea infection was only assessed to a limited extent due to the sampling strategy.

In the flock of strain NL_F725, the C. gallinacea infection preceded an infection with Infectious Laryngotracheitis (ILT) resulting in preventative culling to limit the spread of ILT. Whether a primary infection of $C$. gallinacea enhances infection with other pathogens or whether co-infection might exacerbate the disease outcome, is currently unknown. For C. gallinacea, only co-infections with C. psittaci have been reported in chickens without details about the clinical outcome $(5,45)$. For $C$. psittaci, it has been suggested that coinfections with respiratory pathogens might lead to a more severe disease outcome (46, 47). The effect of co-infection could be a topic for future investigations.

In titration experiments in embryonated eggs, the pathogenicity of C. gallinacea was compared to a virulent $C$. psittaci poultry strain. The infectious dose and mortality in $C$. gallinacea infected eggs was lower compared to C. psittaci infected eggs. Furthermore, although the observations were limited, a small difference in pathogenicity between both C. gallinacea strains was observed. C. gallinacea NL_G47 infection resulted in mortality up to the $10^{-2}$ dilution ( 1 of 5 eggs), while no mortality was observed in the $10^{-2}$ dilution with strain NL_F725 ( 0 of 3 eggs). As follow, this is a first indication of a possible difference in pathogenicity between genetically different $C$. gallinacae strains, but needs to be confirmed due the low number of observations.-

Furthermore, a higher mortality in C. psittaci infected eggs compared to C. gallinacea is in line with findings in available field and experimental studies. In these studies, C. gallinacea infection led to reduced weight gain in chickens and the absence of clinical symptoms, while exposure to a known high virulent $C$. psittaci strain can lead to severe systemic 
infections in chickens and turkeys $(4,10,48,49)$. In contrast, exposure to a less virulent $C$. psittaci strain resulted in mild respiratory symptoms indicating the importance of detailed strain knowledge and infection conditions (48).

The difference in infectious dose and mortality between C. gallinacea and C. psittaci in embryonated eggs might be a result of a shorter development cycle of $C$. psittaci. The development cycle of $C$. gallinacea takes about 60 to 72 hours while that of C. psittaci about 50 hours $(3,50)$. In the experiments, all eggs were harvested at the same time point, which could mean C. psittaci was able to replicate to a higher number of bacteria. The difference in replication time could therefore contribute to the virulence of $C$. psittaci.

To get further insight into the genetic background of $C$. gallinacea in relation to pathogenicity, additional genomic comparisons were performed. Both C. gallinacea isolates were at least $99.4 \%$ identical to C. gallinacea Type strain 08-1274/3, with genetic diversity contained to several distinct chromosomal regions, and had a smaller set of potential virulence genes compared to C. psittaci. However, the question remains if a smaller set of virulence genes is a disadvantage for the particular isolate or species involved and determines the observed difference in pathogenicity. The closest genetic relative of $C$. gallinacea, $C$. avium, also has a reduced set of virulence genes compared to $C$. psittaci, and exhibits the smallest $\mathrm{PZ}$ region of all Chlamydia, but in cases involving pigeons and psittacines infection does lead to clinical signs and mortality $(19,51)$.

Moreover, C. gallinacea does contain all hallmark virulence factors such as Incs, Pmps, T3SS and an intact cytotoxin in the PZ, except in strain JX-1 (12). In addition, C. gallinacea has genes encoding for the well-known T3SS effectors TARP and SINC that play a role in the pathogenesis of Chlamydia spp. In C. psittaci, TARP influences the active uptake in the host cell and SINC targets the nuclear envelope where it is hypothesized to interact with host proteins that control nuclear structure, signaling, chromatin organization, and gene silencing $(52,53)$. Future studies need to confirm if both effectors are indeed secreted in C. gallinacea, with which host proteins they interact, and whether differences in gene expression can be identified that might play a role in pathogenicity.

Based on our current results in embryonated eggs and the genomic comparisons, it is too early to conclude if $C$. gallinacea is a phenotypical commensal. Although less pathogenic than the C. psittaci strains of avian origin, C. gallinacea does possess the hallmark Chlamydia virulence genes, and infection does lead to mortality in embryonated chicken eggs after yolk sac inoculation. Furthermore, there might be small differences in virulence between C. gallinacea strains. Additional pathogenesis studies in chickens, including predisposing conditions such as co-infections, are therefore needed to further elucidate the pathogenic 
potential of $C$. gallinacea and possible strain differences. These future studies will help to assess the importance of this pathogen for poultry industry.

\section{Funding}

This work was supported by the Dutch Ministry of Agriculture, Nature and the Environment (grant WOT-01-002-005.02, WOT-01-01-002-005.13 and KB-21-006-022) and the Australian Research Council Discovery Early Career Research Award (DE190100238) awarded to MJ.

\section{Acknowledgements}

The authors acknowledge Herma Buys, Irene Oud and Marianne Vahl of the WBVR diagnostic lab and for their assistance with PCR tests; Marielle van den Esker for proof reading; Lars Ravesloot for optimizing pictures in the supplementary; Arie Kant and Quillan Dijkstra for technical assistance with Nanopore sequencing; the animal caretakers, Carmen Minnee, Freek Weites and Marc Kranenburg of the department Population Health Sciences, division Farm Animal Health of the faculty of Veterinary Medicine in Utrecht for their assistance in sampling the chickens. The authors would also like to thank Dr. Christiane Schnee from the Friedrich Loeffler Institute in Jena, Germany for providing strain C. gallinacea 08DC65. 


\section{Supporting information}

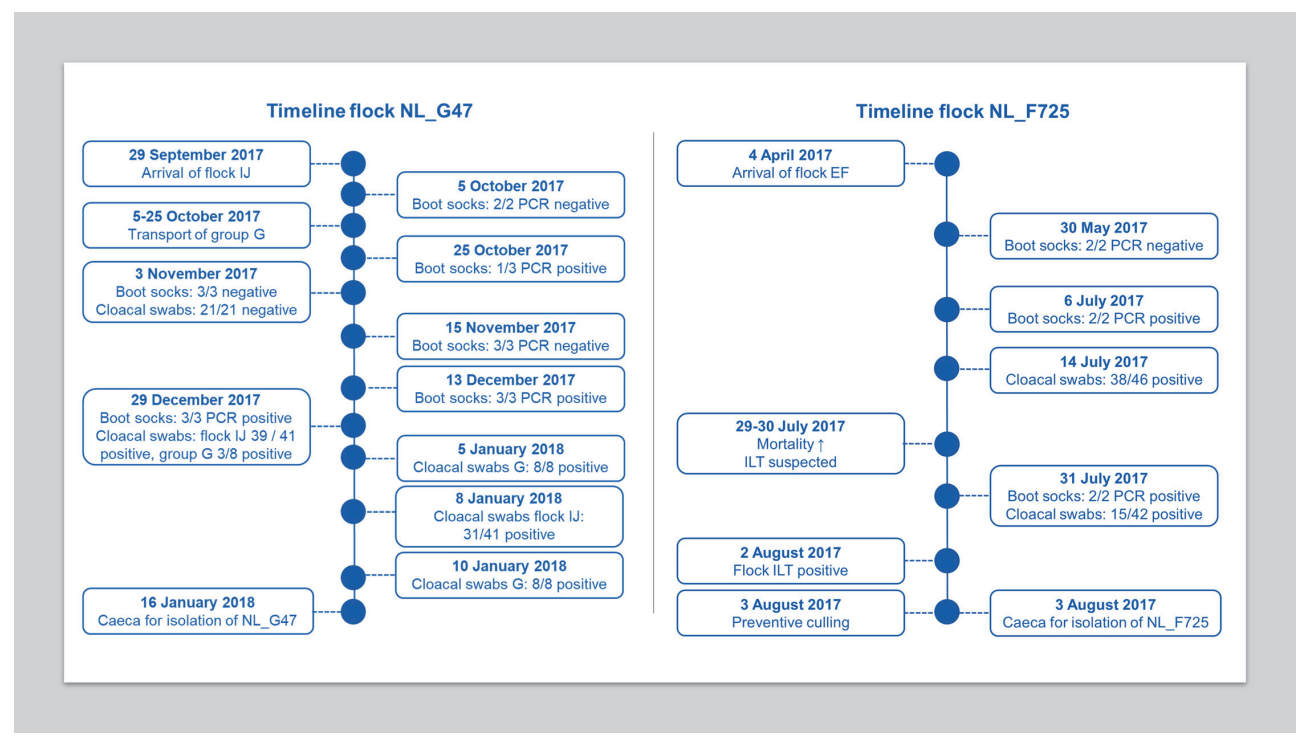

Fig. S1 . Timeline with flock information

In the timeline sampling data are provided of the flocks from which C. gallinacea strain NL_G47 and NL_ F725 could be isolated. All boot sock and cloacal samples were tested with the $23 \mathrm{~S}$ Chlamydiaceae PCR. In the caecal samples for isolation the presence of $C$. gallinacea was confirmed with the C. gallinacea PCR. ILT positivity of flock NL_F725 was confirmed with an antibody ELISA.

A

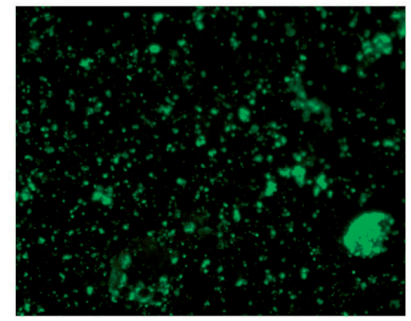

C

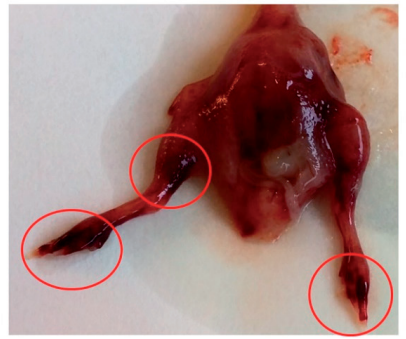

B

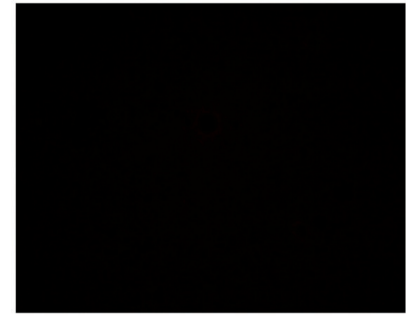

D

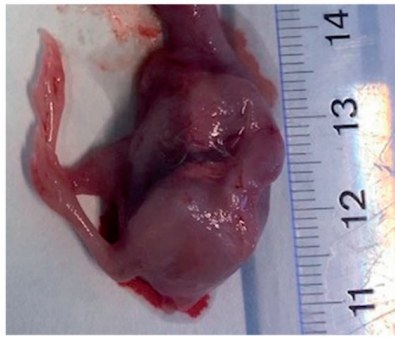

Fig. S2. IFT of the yolk sac membrane and pathologic lesions of the embryo $A$ and $B$ show a positive and a negative IFT result of the yolk sac membrane. $C$ and $D$ show an embryo of an uninfected and a C. gallinacea infected egg with haemorrhages of the toes and upper leg. 


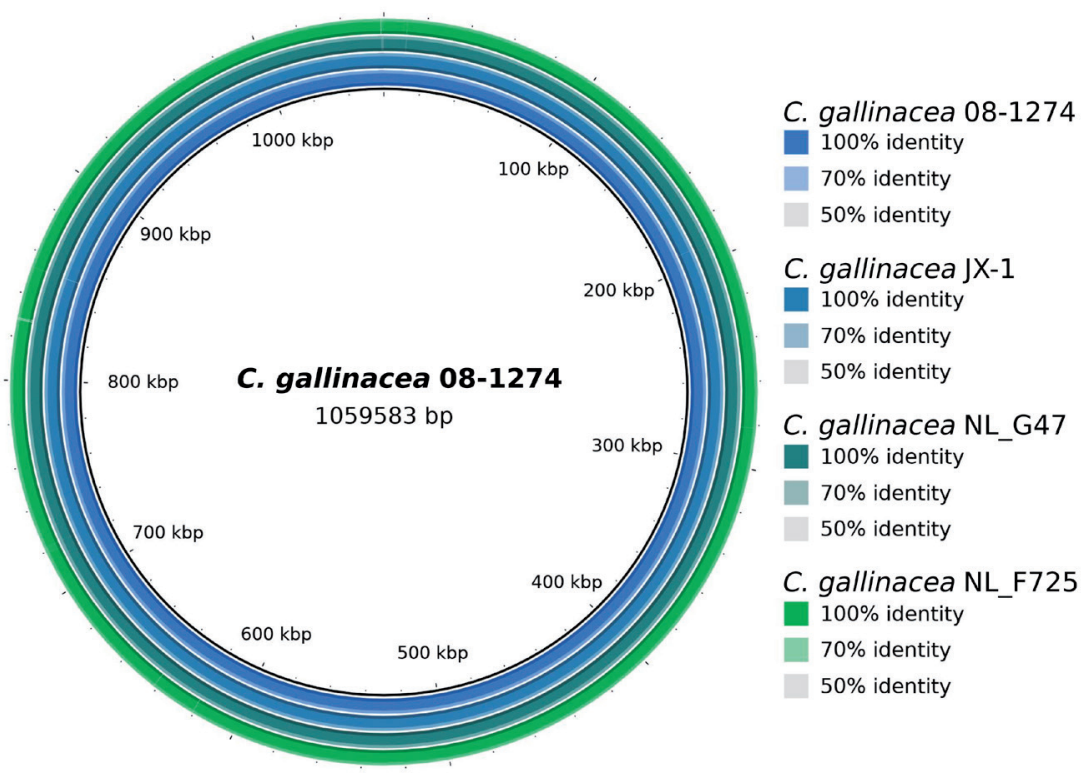

Fig. S3. Genome comparison of four different $\boldsymbol{C}$. gallinacea strains Whole genome BLAST comparisons between four C. gallinacea genomes created with BLAST Ring Image Generator (BRIG)(39) 
Table S1. Results of titration experiments.

In the table the data from all the separate titration experiments were included.

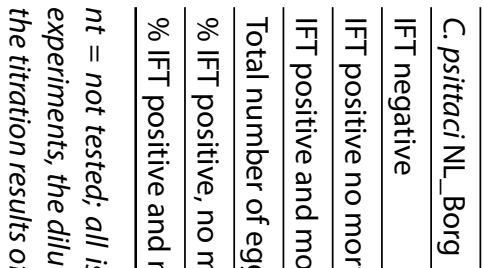

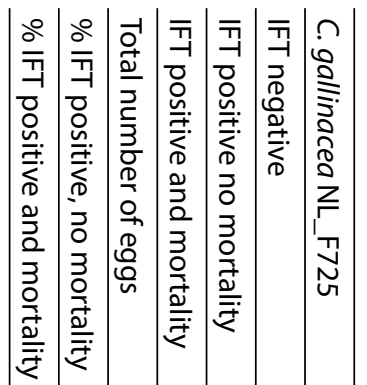

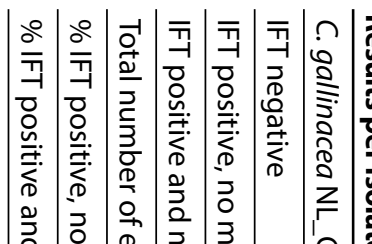



定

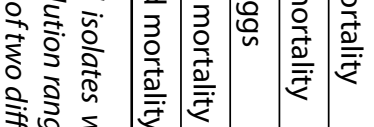

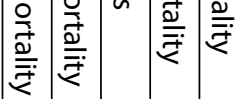

$\frac{0}{9} \stackrel{\frac{0}{2}}{\frac{1}{0}}$

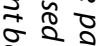

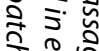

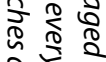

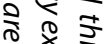





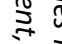

วิ

웛

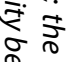

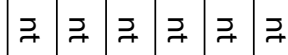

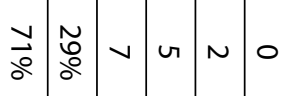

독

帝

है

구 구 굼구 구 구

ə ఫ

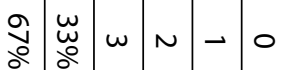

䓌

₹

各

유

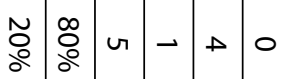

察

ริ ร)

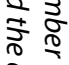

ริ

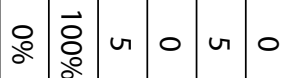

유일

察

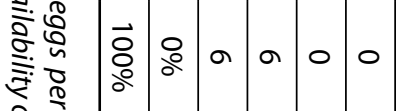

융

○

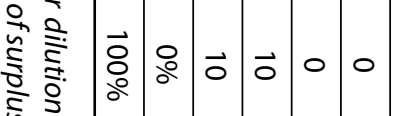

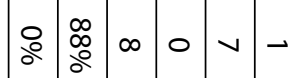

율

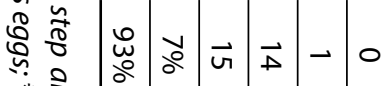

율

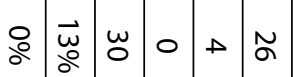
$* \frac{1}{2}$

ㅇำ

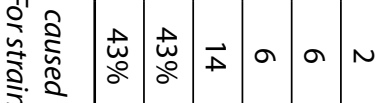

a

유

$\cap \stackrel{0}{2}$

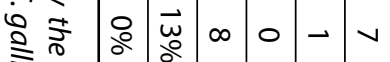

감가 감카 감가

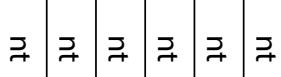

ริ่

\begin{tabular}{lll|l|l|l}
0 & 0 & 0 & 0 \\
5 & 0 & $u$ & 0 & 0 & $u$
\end{tabular}

$\stackrel{\circ}{\mathrm{V}}$

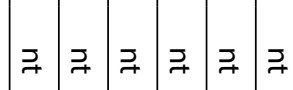

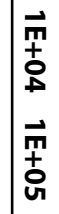

家

㝑

宣

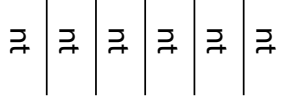


Data S1. Background of flocks. The file provides information about the origin of the flocks, the number of chickens per pen, the breed and the date of hatch and arrival. The file can be found at: https://static-content.springer.com/esm/art\%3A10.1038\%2 Fs41598-021-95966-9/MediaObjects/41598 202195966 MOESM1 ESM.xIsx

\section{Data S2. Details of loci with sequence variation in C. gallinacea.}

The file can be found at: https://static-content.springer.com/esm/art\%3A10.1038\%2 Fs41598-021-95966-9/MediaObjects/41598 202195966 MOESM2 ESM.xIsx

Data S3. Comparison of translated CDSs. Results of the local alignment approach in which all translated CDSs of C. gallinacea 08-1274/3 and C. psittaci NJ1, and vice versa were compared to each other to identify regions with less or no homology. The file can be found at: $\quad$ https://static-content.springer.com/esm/art\%3A10.1038\%2Fs41598-021-95966-9/ MediaObjects/41598 202195966 MOESM3 ESM.xIsx

Data S4. Unique CDSs. List of CDS for which no homologue could be identified in C. gallinacea or C. psittaci. These data are graphically depicted in Fig. 4B.

The file can be found at: https://static-content.springer.com/esm/art\%3A10.1038\%2 Fs41598-021-95966-9/MediaObjects/41598 202195966 MOESM4 ESM.xIsx

\section{Data S5. Results of analysis of predicted T3SS effectors.}

The file can be found at: https://static-content.springer.com/esm/art\%3A10.1038\%2 Fs41598-021-95966-9/MediaObjects/41598_2021_95966_MOESM5_ESM.xIsx

Data S6. MLST and rMLST data. In the file isolates used for rMLST and MLST including provenance and allelic profile data are listed with their allele numbers.

The file can be found at: https://static-content.springer.com/esm/art\%3A10.1038\%2 Fs41598-021-95966-9/MediaObjects/41598_2021_95966_MOESM6_ESM.xIsx 


\section{References}

1. Sachse K, Laroucau K, Vanrompay D. Avian Chlamydiosis. Current Clinical Microbiology Reports. 2015;2(1):10-21.

2. Kaleta EF, Taday EM. Avian host range of Chlamydophila spp. based on isolation, antigen detection and serology. Avian pathology : journal of the WVPA. 2003;32(5):435-61.

3. Harkinezhad T, Geens T, Vanrompay D. Chlamydophila psittaci infections in birds: a review with emphasis on zoonotic consequences. Veterinary microbiology. 2009;135(1-2):68-77.

4. Guo W, Li J, Kaltenboeck B, Gong J, Fan W, Wang C. Chlamydia gallinacea, not C. psittaci, is the endemic chlamydial species in chicken (Gallus gallus). Scientific reports. 2016;6:19638.

5. Hulin V, Oger S, Vorimore F, Aaziz R, de Barbeyrac B, Berruchon J, et al. Host preference and zoonotic potential of Chlamydia psittaci and C. gallinacea in poultry. Pathogens and disease. 2015;73(1):1-11.

6. Li L, Luther M, Macklin K, Pugh D, Li J, Zhang J, et al. Chlamydia gallinacea: a widespread emerging Chlamydia agent with zoonotic potential in backyard poultry. Epidemiology and infection. 2017;145(13):2701-3.

7. Stokes HS, Martens JM, Chamings A, Walder K, Berg ML, Segal Y, et al. Identification of Chlamydia gallinacea in a parrot and in free-range chickens in Australia. Aust Vet J. 2019;97(10):398-400.

8. Li J, Guo W, Kaltenboeck B, Sachse K, Yang Y, Lu G, et al. Chlamydia pecorum is the endemic intestinal species in cattle while C. gallinacea, C. psittaci and C. pneumoniae associate with sporadic systemic infection. Veterinary microbiology. 2016;193:93-9.

9. Laroucau K, Vorimore F, Aaziz R, Berndt A, Schubert E, Sachse K. Isolation of a new chlamydial agent from infected domestic poultry coincided with cases of atypical pneumonia among slaughterhouse workers in France. Infection, genetics and evolution : journal of molecular epidemiology and evolutionary genetics in infectious diseases. 2009;9(6):1240-7.

10. Heijne M, van der Goot JA, Fijten H, van der Giessen JW, Kuijt E, Maassen CBM, et al. A cross sectional study on Dutch layer farms to investigate the prevalence and potential risk factors for different Chlamydia species. PloS one. 2018;13(1):e0190774.

11. You J, Wu Y, Zhang X, Wang X, Gong J, Zhao Z, et al. Efficient fecal-oral and possible vertical, but not respiratory, transmission of emerging Chlamydia gallinacea in broilers. Veterinary microbiology. 2019;230:90-4.

12. Guo W, Jelocnik M, Li J, Sachse K, Polkinghorne A, Pannekoek Y, et al. From genomes to genotypes: molecular epidemiological analysis of Chlamydia gallinacea reveals a high level of genetic diversity for this newly emerging chlamydial pathogen. BMC genomics. 2017;18(1):949.

13. Holzer M, Barf LM, Lamkiewicz K, Vorimore F, Lataretu M, Favaroni A, et al. Comparative Genome Analysis of 33 Chlamydia Strains Reveals Characteristic Features of Chlamydia Psittaci and Closely Related Species. Pathogens. 2020;9(11).

14. Kilkenny C, Browne WJ, Cuthill IC, Emerson M, Altman DG. Improving bioscience research reporting: The ARRIVE guidelines for reporting animal research. J Pharmacol Pharmacother. 2010;1(2):94-9. 
15. Kuo CC, Stephens, R.S., Bavoil, P.M. and Kaltenbroeck, B. Chlamydia, Bergey's Manual of Systematics of Archae and Bacteria2015.

16. Anonymous. Avian Chlamydiosis. OIE Terrestrial Manual 20182018. p. 783-95.

17. Andersson C, Gripenland J, Johansson J. Using the chicken embryo to assess virulence of Listeria monocytogenes and to model other microbial infections. Nature protocols. 2015;10(8):1155-64.

18. Storz J. Chlamydia and Chlamydia-Induced Diseases: Charles C Thomas; 1971.

19. Kik M, Heijne M, ljzer J, Grinwis G, Pannekoek Y, Gröne A. Fatal <em>Chlamydia avium</em> Infection in Captive Picazuro Pigeons, the Netherlands. Emerging Infectious Disease journal. 2020;26(10):2520.

20. Branley J, Bachmann NL, Jelocnik M, Myers GS, Polkinghorne A. Australian human and parrot Chlamydia psittaci strains cluster within the highly virulent $6 \mathrm{BC}$ clade of this important zoonotic pathogen. Scientific reports. 2016;6:30019.

21. SPEARMAN C. THE METHOD OF 'RIGHT AND WRONG CASES' ('CONSTANT STIMULI') WITHOUT GAUSS'S FORMULAE. British Journal of Psychology, 1904-1920. 1908;2(3):227-42.

22. Kärber G. Beitrag zur kollektiven Behandlung pharmakologischer Reihenversuche. NaunynSchmiedebergs Archiv für experimentelle Pathologie und Pharmakologie. 1931;162(4):480-3.

23. Ehricht R, Slickers P, Goellner S, Hotzel H, Sachse K. Optimized DNA microarray assay allows detection and genotyping of single PCR-amplifiable target copies. Molecular and cellular probes. 2006;20(1):60-3.

24. Souvorov A, Agarwala R, Lipman DJ. SKESA: strategic k-mer extension for scrupulous assemblies. Genome Biol. 2018;19(1):153.

25. Tatusova T, DiCuccio M, Badretdin A, Chetvernin V, Nawrocki EP, Zaslavsky L, et al. NCBI prokaryotic genome annotation pipeline. Nucleic acids research. 2016;44(14):6614-24.

26. Kumar S, Stecher G, Tamura K. MEGA7: Molecular Evolutionary Genetics Analysis Version 7.0 for Bigger Datasets. Mol Biol Evol. 2016;33(7):1870-4.

27. Saitou N, Nei M. The neighbor-joining method: a new method for reconstructing phylogenetic trees. Mol Biol Evol. 1987;4(4):406-25.

28. Felsenstein J. Confidence Limits on Phylogenies: An Approach Using the Bootstrap. Evolution. 1985;39(4):783-91.

29. Tamura K, Nei M, Kumar S. Prospects for inferring very large phylogenies by using the neighborjoining method. Proceedings of the National Academy of Sciences of the United States of America. 2004;101(30):11030-5.

30. Jolley KA, Bliss CM, Bennett JS, Bratcher HB, Brehony C, Colles FM, et al. Ribosomal multilocus sequence typing: universal characterization of bacteria from domain to strain. Microbiology (Reading). 2012;158(Pt 4):1005-15.

31. Pannekoek Y, Morelli G, Kusecek B, Morre SA, Ossewaarde JM, Langerak AA, et al. Multi locus sequence typing of Chlamydiales: clonal groupings within the obligate intracellular bacteria Chlamydia trachomatis. BMC microbiology. 2008;8:42. 
32. Goris J, Konstantinidis KT, Klappenbach JA, Coenye T, Vandamme P, Tiedje JM. DNA-DNA hybridization values and their relationship to whole-genome sequence similarities. Int J Syst Evol Microbiol. 2007;57(Pt 1):81-91.

33. Jain C, Rodriguez RL, Phillippy AM, Konstantinidis KT, Aluru S. High throughput ANI analysis of 90K prokaryotic genomes reveals clear species boundaries. Nat Commun. 2018;9(1):5114.

34. Gurevich A, Saveliev V, Vyahhi N, Tesler G. QUAST: quality assessment tool for genome assemblies. Bioinformatics. 2013;29(8):1072-5.

35. Seeman T. Snippy: fast bacterial variant calling from NGS reads https://github.com/tseemann/ snippy [30 December 2018].

36. Rozas J, Ferrer-Mata A, Sanchez-DelBarrio JC, Guirao-Rico S, Librado P, Ramos-Onsins SE, et al. DnaSP 6: DNA Sequence Polymorphism Analysis of Large Data Sets. Mol Biol Evol. 2017;34(12):3299-302.

37. Eichinger V, Nussbaumer T, Platzer A, Jehl MA, Arnold R, Rattei T. EffectiveDB--updates and novel features for a better annotation of bacterial secreted proteins and Type III, IV, VI secretion systems. Nucleic acids research. 2016;44(D1):D669-74.

38. Moller S, Croning MD, Apweiler R. Evaluation of methods for the prediction of membrane spanning regions. Bioinformatics. 2001;17(7):646-53.

39. Alikhan NF, Petty NK, Ben Zakour NL, Beatson SA. BLAST Ring Image Generator (BRIG): simple prokaryote genome comparisons. BMC genomics. 2011;12:402.

40. Sullivan MJ, Petty NK, Beatson SA. Easyfig: a genome comparison visualizer. Bioinformatics. 2011;27(7):1009-10.

41. Buchfink $B$, Xie $C$, Huson DH. Fast and sensitive protein alignment using DIAMOND. Nat Methods. 2015;12(1):59-60.

42. Wickham H, Averick, M., Bryan, J., Chang, W., D’Agostino McGowan, L., Francois, R., Grolemund, G., Hayes, A., Henry, L., Hester, J., Kuhn, M., Lin Pedersen, T., Miller, E., Bache, S.M., Muller, K., Ooms, J., Robinson D., Paige Seidel, D., Spinu, V., Takahashi, K., Vaughan,. D., Wilke, C., Woo, K., Yutani, H. Welcome to the Tidyverse. Journal of Open Source Software. 2019;4.

43. Team RC. A language and environment for statistical computing. Vienna, Austria: R Foundation for Statistical Computing; 2019.

44. Knittler MR, Berndt A, Bocker S, Dutow P, Hanel F, Heuer D, et al. Chlamydia psittaci: new insights into genomic diversity, clinical pathology, host-pathogen interaction and anti-bacterial immunity. Int J Med Microbiol. 2014;304(7):877-93.

45. Donati M, Laroucau K, Guerrini A, Balboni A, Salvatore D, Catelli E, et al. Chlamydiosis in Backyard Chickens (Gallus gallus) in Italy. Vector borne and zoonotic diseases. 2018;18(4):222-5.

46. Chu J, Zhang Q, Zhang T, Han E, Zhao P, Khan A, et al. Chlamydia psittaci infection increases mortality of avian influenza virus H9N2 by suppressing host immune response. Scientific reports. 2016;6:29421.

47. Chu J, Zhang Q, Zuo Z, El-Ashram S, Guo Y, Zhao P, et al. Co-infection of Chlamydia psittaci with H9N2, ORT and Aspergillus fumigatus contributes to severe pneumonia and high mortality in SPF chickens. Scientific reports. 2017;7(1):13997. 
48. Yin L, Lagae S, Kalmar I, Borel N, Pospischil A, Vanrompay D. Pathogenicity of low and highly virulent Chlamydia psittaci isolates for specific-pathogen-free chickens. Avian diseases. 2013;57(2):242-7.

49. Page LA. Experimental Ornithosis in Turkeys. Avian diseases. 1959;3(1):51-66.

50. Sachse K, Laroucau K, Riege K, Wehner S, Dilcher M, Creasy HH, et al. Evidence for the existence of two new members of the family Chlamydiaceae and proposal of Chlamydia avium sp. nov. and Chlamydia gallinacea sp. nov. Systematic and applied microbiology. 2014;37(2):79-88.

51. Laroucau K, Popelin-Wedlarski F, Roux A, Aaziz R, Vorimore F, Lagourette P, et al. Multiple cases of Chlamydia avium infection in captive psittacines. Avian diseases. 2020.

52. Radomski N, Einenkel R, Muller A, Knittler MR. Chlamydia-host cell interaction not only from a bird's eye view: some lessons from Chlamydia psittaci. FEBS Lett. 2016;590(21):3920-40.

53. Mojica SA, Hovis KM, Frieman MB, Tran B, Hsia RC, Ravel J, et al. SINC, a type III secreted protein of Chlamydia psittaci, targets the inner nuclear membrane of infected cells and uninfected neighbors. Mol Biol Cell. 2015;26(10):1918-34. 
CHAPTER 4 


\section{Pathogenicity of Chlamydia gallinacea in chickens after oral inoculation}

Marloes Heijne ${ }^{*}$, Jeanet van der Goot ${ }^{2}$, Herma Buys², Annemieke Dinkla' ${ }^{1}$, Hendrik Jan Roest ${ }^{3}$, Lucien van Keulen', Ad Koets ${ }^{1,4}$

\footnotetext{
${ }^{1}$ Department of Bacteriology, Host-Pathogen Interaction and Diagnostics Development, Wageningen Bioveterinary Research, Lelystad, the Netherlands

2 Department of Diagnostics and Crisis Organisation, Wageningen Bioveterinary Research, Lelystad, the Netherlands

${ }^{3}$ Directorate Animal Supply Chain and Animal Welfare, Ministry of Agriculture, Nature and Food Quality, The Hague, the Netherlands

${ }^{4}$ Department of Population Health Sciences, Faculty of Veterinary Medicine, Utrecht

University, Utrecht, the Netherlands
}

Vet Microbiol. 2021 Aug;259:109166. doi: 10.1016/j.vetmic.2021.109166. Epub 2021 


\section{Abstract}

Chlamydia gallinacea is a recently discovered and widespread obligate intracellular bacterium in chickens. In chickens, infections appear to be asymptomatic, but can result in reduced weight gain in broilers. Molecular typing revealed $C$. gallinacea is genetically diverse which might lead to differences in pathogenic potential between strains. However, studies about the pathogenesis of different C. gallinacea strains are still limited. In this study, the pathogenesis of C. gallinacea strain NL_G47 was investigated in three consecutive animal experiments. The first experiment served as a pilot in which a maximum culturable dose was administered orally to 13 chickens. Excretion of chlamydial DNA in cloacal swabs was measured during 11 days post infection, but no clinical signs were observed. The second and third experiment were a repetition of the first experiment, but now chickens were sacrificed at consecutive time points to investigate tissue dissemination of $C$. gallinacea. Again excretion of chlamydial DNA in cloacal swabs was detected and no clinical signs were observed in line with the results of the first experiment. PCR and immunohistochemistry of tissue samples revealed $C$. gallinacea infected the epithelium of the jejunum, ileum and caecum. Furthermore, $C$. gallinacea could be detected in macrophages in the lamina propria and in follicular dendritic cells (FDCs) of the B cell follicles in the caecal tonsil. Results of serology showed a systemic antibody response from day seven or eight and onward in all three experiments. The experiments with strain NL_G47 confirmed observations from field studies that $C$. gallinacea infection does not result in acute clinical disease and mainly resides in the epithelium of the gut. Whether the presence of $C$. gallinacea results in chronic persistent infections with long term and less obvious health effects in line with observations on other infections caused by Chlamydiae, needs further investigation. 


\section{Introduction}

Chlamydia gallinacea is an obligate intracellular bacterium belonging to the family of Chlamydiaceae. This family comprises important pathogens including the zoonotic Chlamydia psittaci and the strictly human pathogen Chlamydia trachomatis. Since the proposal of C. gallinacea as a new species in 2014, high prevalences are reported in poultry in different countries around the world (1-4). In poultry, transmission occurs via the fecaloral route (5). Infections appear to be asymptomatic, although yolk sac inoculation of embryonated chicken eggs caused mortality and experimental infection of broilers resulted in reduced weight gain $(4,6)$. Zoonotic transmission of $C$. gallinacea has been considered, but there is no definite proof $(2,5,7)$.

Genomic studies revealed C. gallinacea has the hallmark Chlamydia virulence genes, although to a lesser number than $C$. psittaci $(6,8,9)$. However, the relation between the number and type of virulence genes and the phenotypical outcome is not straightforward. Chlamydia avium, for example, the closest relative of $C$. gallinacea, probably has the lowest number of virulence associated genes compared to other chlamydial species (9), but infections are associated with clinical disease and mortality in pigeons and parrots $(1,10)$. Furthermore, molecular typing provided evidence for substantial genetic diversity among C. gallinacea strains, which might result in differences in pathogenicity (8). Therefore, further research into the pathogenicity of various $C$. gallinacea strains is needed.

Here, we investigated the primary pathogenicity of $C$. gallinacea strain NL_G47 in chickens. Previous Multi Locus Sequence Typing (MLST) revealed strain NL_G47 has an unique sequence type (ST 280) and forms a well-supported clade with Type strain 08-1274/3(6). Furthermore, strain NL_G47 is genetically different from strain JX-1 which was used in other published experimental studies $(4,5)$. Strain NL_G47 was isolated from an asymptomatic laying hen from a Dutch flock in 2018, and, after inoculation in the yolk sac of embryonated chicken eggs, mortality was observed (6). In the present study, chickens were inoculated orally with NL_G47 and shedding was measured in throat and cloacal swabs during 11 days post infection. In addition, tissue dissemination was investigated through sequentially sacrificing of animals and blood was collected to measure a serologic response. The results from this study will help to assess if $C$. gallinacea infection causes acute disease in chickens and if $C$. gallinacea should be considered a threat to poultry health. 


\section{Materials and methods}

\section{Ethical statement}

The animal experiment was conducted in accordance with the national regulations on animal experimentation. The project license was approved by the Dutch Central Authority for Scientific Procedures on Animals (CCD) (permit number AVD4010020173926).

\section{Inoculum}

Chlamydia gallinacea NL_G47 was isolated from caecal material from a clinically healthy laying hen rom a Dutch flock in 2018 as described earlier. The isolate was passaged three times in the yolk sac of SPF chicken eggs and stored at $-80^{\circ} \mathrm{C}$ as a $20 \%$ yolk sac suspension in Sucrose Phosphate Glutamate (SPG) until inoculation. The infectious dose of the suspension was determined by egg titration experiments and expressed as the Egg Infectious Dose $50\left(\operatorname{EID}_{50}\right)(6)$.

\section{Animals and housing}

A total of 39 five-week-old Specified Pathogen Free (SPF) White Leghorn hens were obtained from MSD Animal Health (Boxmeer, the Netherlands). Chlamdiaceae are not included in standard SPF testing, therefore three additional drag swabs of the incubators of the parent flock were collected. All drag swabs tested PCR negative for Chlamydia spp. At arrival a pooled fecal sample taken from the transport boxes of the five-week old hens also tested PCR negative for Chlamydia spp. All chickens had a 6-day acclimatization period prior to inoculation.

At arrival the hens were housed as a group on sawdust bedding in temperature-controlled rooms under optimal light conditions and humidity. Feed and water were provided ad libitum. The experiment was performed in biosafety level 2 (BSL 2) facilities at Wageningen Bioveterinary Research (WBVR, Lelystad, the Netherlands).

\section{Experimental design}

Three subsequent experiments were performed with thirteen chickens per experiment. In every experiment chickens were assigned a number randomly. The first experiment was a pilot experiment to test the inoculation route and dose. If shedding could be shown and the experiment would not lead to severe clinical signs or mortality, the second and third experiment would be repeated with the same dose and inoculation route as the first experiment.

In the second and third experiment chickens were sequentially sacrificed: three chickens at day zero (before inoculation), three at day four, three at day eight and four at day 11 after inoculation. The chickens that were sacrificed at day zero served as a negative control group.

In every separate experiment inoculation was performed orally with a $1 \mathrm{ml}$ syringe and an oral gavage needle. All chickens, except the control groups were inoculated with 0.5 
$\mathrm{ml}$ of a $20 \%$ yolk suspension in SPG with an infectious dose of $10^{5.2}$ EID $_{50}$ per bird. The inoculation dose was confirmed by back-titration, for each experiment and the infectious dose was found to be within a range of $0.7 \log 10 \mathrm{EID}_{50} / \mathrm{mL}$ of the initial dose.

Clinical signs were recorded daily according to a clinical scoring card (Table S1). Throat and cloacal swabs were collected daily. Serum samples were collected at day zero, day seven and at euthanasia at day four, eight or 11. A timeline of the experiments including sampling moments is given in Fig. 1. All samples at day zero were collected prior to inoculation to confirm the absence of a current Chlamydia infection. All experiments finished 11 days after inoculation.

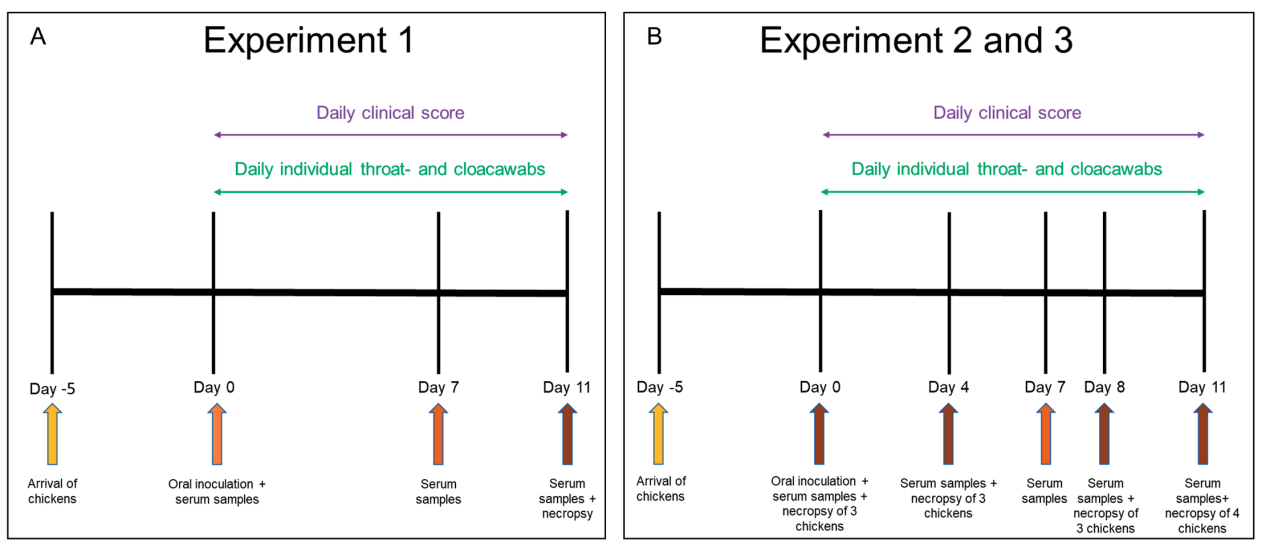

Fig. 1. Timeline of experiments with sampling moments

The chickens in the first experiment were euthanized by intraperitoneal administration of one $\mathrm{ml}$ pentobarbital (Euthasol 50\% solution, AST Farma, Oudewater, the Netherlands). In the second and third experiment the chickens were euthanized by maximum blood collection via heart puncture under generalised anesthesia by intramuscular injection of a mixture of $0.3 \mathrm{ml} / \mathrm{kg}$ ketamine (Ketamine 10\% Alfasan) and $0.5 \mathrm{ml} / \mathrm{kg}$ xylazine (Sedamun, Dechra).

\section{Necropsy}

In the first experiment all animals were sacrificed at day 11 and samples were collected from airsac, lung, liver, spleen, ileum, caecum and colon. In the second and third experiment all carcasses were opened on a clean plastic sheet which was replaced after each necropsy. To prevent cross contamination new sterile instruments and petridishes were used for every tissue sample. Tissue samples (approximately $0,5 \mathrm{~cm}^{3}$ ) were collected from the airsac, lung, liver, spleen, kidney, esophagus, proventriculus, ventriculus, duodenum, jejunum, ileum, caecum, caecal tonsil and colon. Samples for PCR were collected in $1 \mathrm{ml} \mathrm{SPG}$ in Lysing Matrix D tubes (MP Biomedicals) and ribolysed ( $2 \times 20 \mathrm{sec}$ at $4 \mathrm{~m} / \mathrm{sec}$ ) before storage at -80 
${ }^{\circ} \mathrm{C}$. Tissue samples for histology and immunohistochemistry were collected in $10 \%$ neutral buffered formalin and routinely processed into paraffin blocks. In the third experiment additional tissue samples were collected from jejunum, ileum, caecum and caecal tonsil, embedded in OCT compound using cryomoulds (TissueTek ${ }^{\circledR}$, Sakura Finetek, USA), snap frozen with liquid nitrogen and stored at $-80^{\circ} \mathrm{C}$.

\section{PCR analyses}

Swabs were suspended in $1.5 \mathrm{ml}$ PBS and thoroughly vortexed. From swab or tissue suspension, $200 \mu \mathrm{l}$ was used for DNA extraction. DNA extraction was performed with a MagNA Pure LC total Nucleic Acid Isolation kit in the MagNA Pure ${ }^{\circledR}$ system (Roche Diagnostics, Almere, the Netherlands). DNA was tested with a Chlamydiaceae PCR targeting the $23 \mathrm{~S}$ rRNA $(2,11)$.

\section{Histology and immunohistochemistry}

Frozen and formalin fixed tissue samples were cut into $4 \mu \mathrm{m}$ sections and collected on positively charged glass slides (SuperfrostPlus ${ }^{\circledast}$, Thermo Scientific). Frozen samples were fixed for 10 minutes in acetone and air dried. Sections were then stained with haematoxylin-eosin (HE) or immunostained with a polyclonal anti-Chlamydia antibody (LifeSpan BioSciences, Cat\# LS-C85741-1000, RRID:AB_1813851) or a monoclonal antiChlamydia antibody (MyBioSource, Cat\# MBS830551). Epitope retrieval of the formalin fixed sections consisted of proteolysis induced epitope retrieval for the polyclonal antibody $\left(0,1 \%\right.$ protK in TBS for $30 \mathrm{~min}$ at $\left.37^{\circ} \mathrm{C}\right)$ and heat induced epitope retrieval (citrate buffer, $\mathrm{pH} 6.0,121^{\circ} \mathrm{C}$ for $5 \mathrm{~min}$ ) for the monoclonal antibody. Anti-rabbit or anti-mouse HRP conjugated polymer was used as a secondary antibody (Invitrogen, Carlsbad, USA).

Subsequently, formalin fixed sections were incubated for 5 minutes in DAB+ substrate (Dako, Agilent, Santa Clara, USA), counterstained with Mayer's hematoxylin and mounted permanently. Cryo sections were incubated with Alexa Fluor ${ }^{\mathrm{TM}} 488,546$, or 647 tyramide reagent (Invitrogen) and mounted in antifading mounting medium containing DAPI (Vector laboratories, Peterborough, UK). Co-localisation of chlamydial antigen was assesed by double immunofluoresence staining using a mouse anti-chicken monocyt / macrophages monoclonal (Clone KUL01, Southern Biotech, Birmingham, USA) or a mouse anti-chicken FDCs monoclonal (Clone 74.3, WBVR, Lelystad, the Netherlands). Sections were photographed with an Olympus BX51 (fluorescence) microscope equipped with a highresolution digital camera. Monochromatic digital photographs for immunofluorescence were false colored using CellSense ${ }^{\circledast}$ software.

\section{Serology}

Serum samples were tested with an in-house ELISA coated with a commercial mix of Chlamydia abortus and Chlamydia trachomatis antigen (Institut Virion\Serion $\mathrm{GmbH}$, Würzburg, Germany), because specific serological tests for C. gallinacea are currently not 
available. Ninety-six-well microtiter plates (Nunc MaxiSorp ${ }^{\mathrm{TM}}$, Thermo Fisher Scientific, Landsmeer, the Netherlands) were coated overnight at $37^{\circ} \mathrm{C}$ with $100 \mu$ per well with a concentration of $4 \mu \mathrm{g} / \mathrm{ml}$ of each antigen in coating buffer BM112 (WBVR, Lelystad, the Netherlands). Following six washes with $0.05 \%$ Tween $^{\circledast} 80$, the plates were blocked with $190 \mu \mathrm{l}$ per well of $5 \%$ skimmed-milk powder (Campina Elk, the Netherlands) in TBST (BM309, WBVR, Lelystad, the Netherlands) for $60 \mathrm{~min}$ at room temperature (RT). The plates were washed as described above, then $100 \mu$ of chicken serum per well (diluted 1:500 in $5 \%$ skimmed milk powder-TBST) was added and the plates were incubated for $60 \mathrm{~min}$ at $37^{\circ} \mathrm{C}$. After further washing, $100 \mu \mathrm{l}$ of goat anti-chicken $\lg \mathrm{Y}(\mathrm{H}+\mathrm{L})$-HRP (Southern Biotech, Birmingham, USA, diluted 1:6,000 in 5\% skimmed milk powder-TBST) was added per well, and the mixture was incubated for $60 \mathrm{~min}$ at $37^{\circ} \mathrm{C}$. Again six washes with $0.05 \%$ Tween $^{\circledast} 80$ were performed and one wash with Super- $\mathrm{Q}^{\circledR}$ water. Bound antibody was detected with TMB One component HRP Microwell substrate (TMBW-1000-01, SurModics, Minnesota, USA). The reaction was terminated after $10 \mathrm{~min}$ by the addition of $0.5 \mathrm{M}$ sulfuric acid. The optical density (OD) was measured at $450 \mathrm{~nm}$ on a Thermo Labsystems Multiskan RC microplate reader (Thermo Fisher Scientific, Landsmeer, the Netherlands).

Per plate, two plate controls were included with two wells per control. In one control, no serum and no conjugate was added to the wells, in the other control no serum was added. All obtained chicken sera were tested in one batch and the individual OD values were corrected for plate differences by subtracting the mean OD value of the plate control (without serum but with conjugate).

\section{Results}

\section{Clinical signs and shedding}

In the first experiment no clinical signs were observed and shedding was shown in both throat and cloacal swabs (Fig. 2A and D). The second and third experiment were therefore performed with the same inoculation dose and route. During experiment two and three no clinical signs were reported, all chickens appeared clinically healthy at necropsy and no pathological lesions were observed. The PCR results of shedding in throat and cloacal swabs of the second an third experiment are shown in Fig. 2B, E, C and F.

In all three experiments, a similar shedding pattern in both throat and cloacal swabs was observed. Overall shedding was higher in cloacal swabs than in throat swabs. In cloacal swabs shedding increased in the first four to five days and then flattened. 
A

Experiment 1: throat swabs

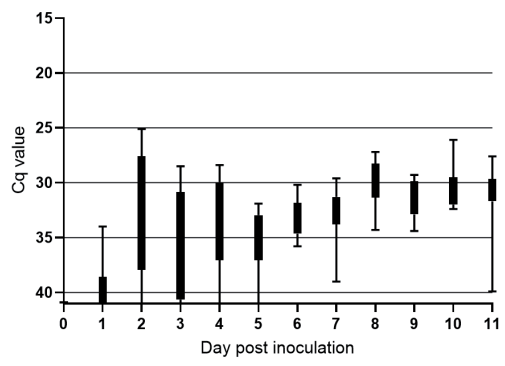

C

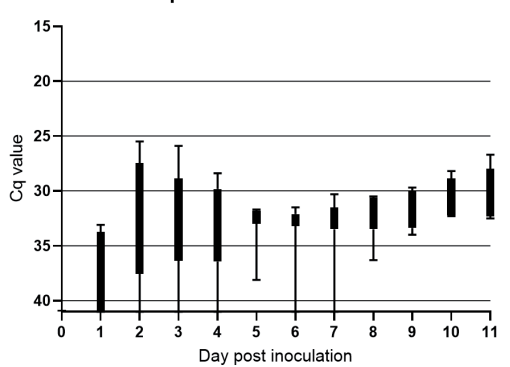

$E$

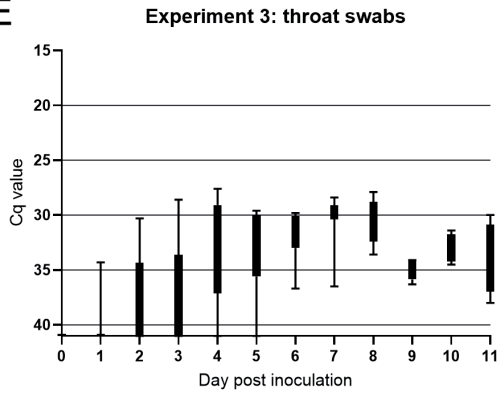

B Experiment 1: cloacal swabs

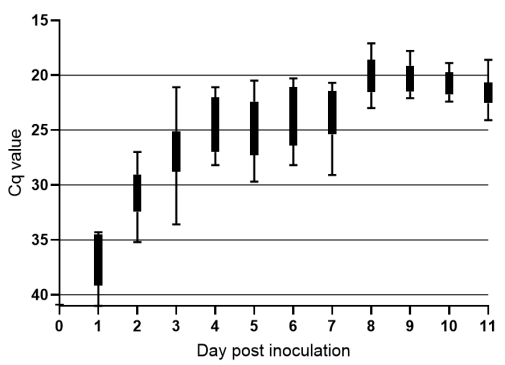

D

Experiment 2: cloacal swabs

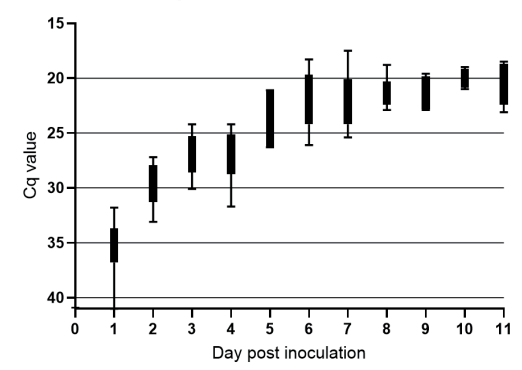

$\mathrm{F}$

Experiment 3: cloacal swabs

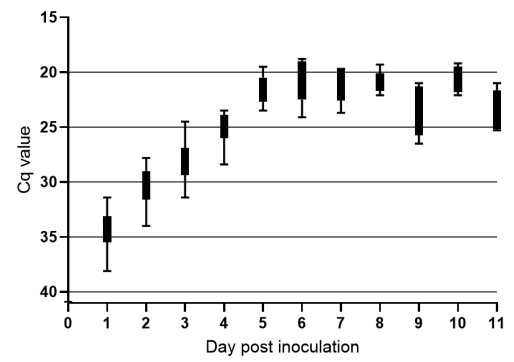

Fig. 2. $P C R$ results of throat $(A, C, E)$ and cloacal swabs $(B, D, F)$

The results are shown per experiment. On the $Y$-axis the cycle treshold $(\mathrm{Cq})$ value is depicted. The $Y$-axis has been rotated and $C q$ values $>40$ are shown as $C t 41$. The whiskers plot down to the smallest value and up to the largest and the box extends from the 25th to 75th percentile. In A and B every day post infection (dpi) at the $X$-axis shows the $P C R$ results of 13 chickens. In $C, D, E$ and $F$ dpi 0 shows the results of 13 chickens, dpi 1-4 of 10 chickens, dpi 5-8 of 7 chickens and dpi 9-11 of 4 chickens. 


\section{Dissemination in the gastro-intestinal tract}

In Fig. 3, PCR results of dissemination of C. gallinacea per timepoint in the gastrointestinal tract in the second and third experiment are depicted. The results of experiment 2 and 3 show that the load of chlamydiae increases towards the more distal parts of the gut, i.e. jejunum, ileum, caecum and colon. The load also increases in time from day 4 to day 8 in all sample types, and appears to be in the same range at day 8 and day 11 .

A

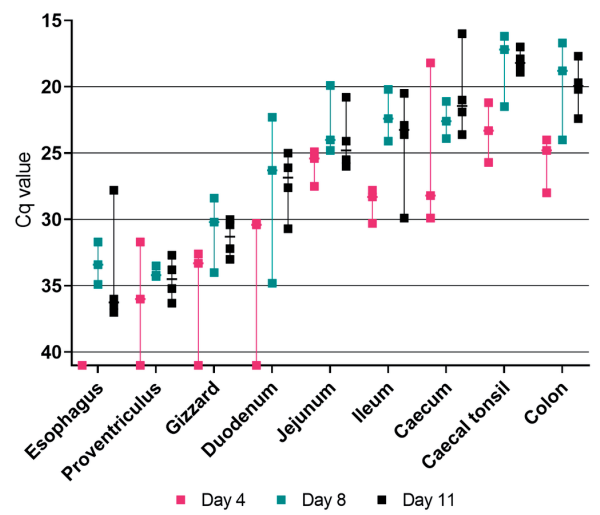

B

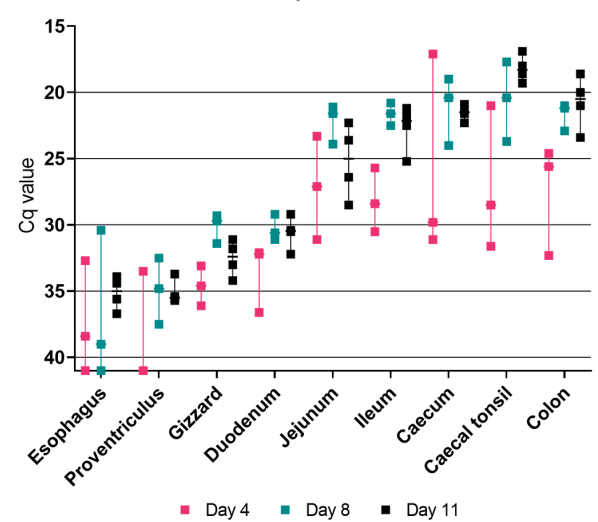

Fig. 3. PCR results of samples from the gastrointestinal tract

A shows the results of experiment 2 and $B$ of experiment 3. Per timepoint the median and range of the individual samples are shown. The results of day 0 are not presented as all samples tested $P C R$ negative. On the $Y$-axis the cycle treshold $(\mathrm{Cq})$ value is depicted. The axis has been rotated and Cq values $>40$ are shown as $C q 41$.

In the HE sections of the gut, chlamydiae were not clearly discernible in any of the tissues. In addition, no inflammatory response was seen in the lamina propria or submucosa (Fig. $4 \mathrm{~A}$ and $\mathrm{B}$ ). However, using immunohistochemistry, chlamydial antigen was detected from day 4 onward in the epithelium of the jejunum, ileum and caecum but not in the colon (Fig. 4A). Chlamydiae were seen in rounded structures at the luminal side of the cells (inclusion bodies) or located diffusely in the cytoplasm (Fig. 4B). 



Fig. 4. Histology and immunohistochemistry of the ileum and caecum

$A$ and B: IHC staining for Chlamydia in the ileum at day 4 post infection Bacteria are clearly visible in the epithelium either as apical located inclusion bodies or diffusely present in the cytoplasm. A bar $=100 \mu \mathrm{m}$, $B$ bar $=20 \mu \mathrm{m}$. C and D:IHC staining for Chlamydia in the caecal tonsil at 11 days post infection increased bacterial load in the epithelium compared to day 4 resulting in an almost continuous lining of the gut lumen. Chlamydial antigen is also present within single cells in the lamina propria (arrow) and in the lymphoid follicles (arrowhead). C. bar $=200 \mu \mathrm{m}$, D. bar $=100 \mu \mathrm{m}$

At day 8 and 11 after infection, the number of epithelial cells that stained positively for chlamydia had increased. Chlamydiae were most abundant in the epithelium of the caecum and caecal tonsil (Fig. 5C and D). In addition to the staining of the epithelium, chlamydial antigen was seen in single cells within the lamina propria and within the lymphoid follicles of the ileum and caecal tonsil.

Double immunostaining for chlamydial antigen and chicken cell markers showed that chlamydia co-localized with follicular dendritic cells in the lymphoid follicles (Fig. 5 A-C) and mononuclear phagocytes within the lamina propria (Fig. 5 D-F). 

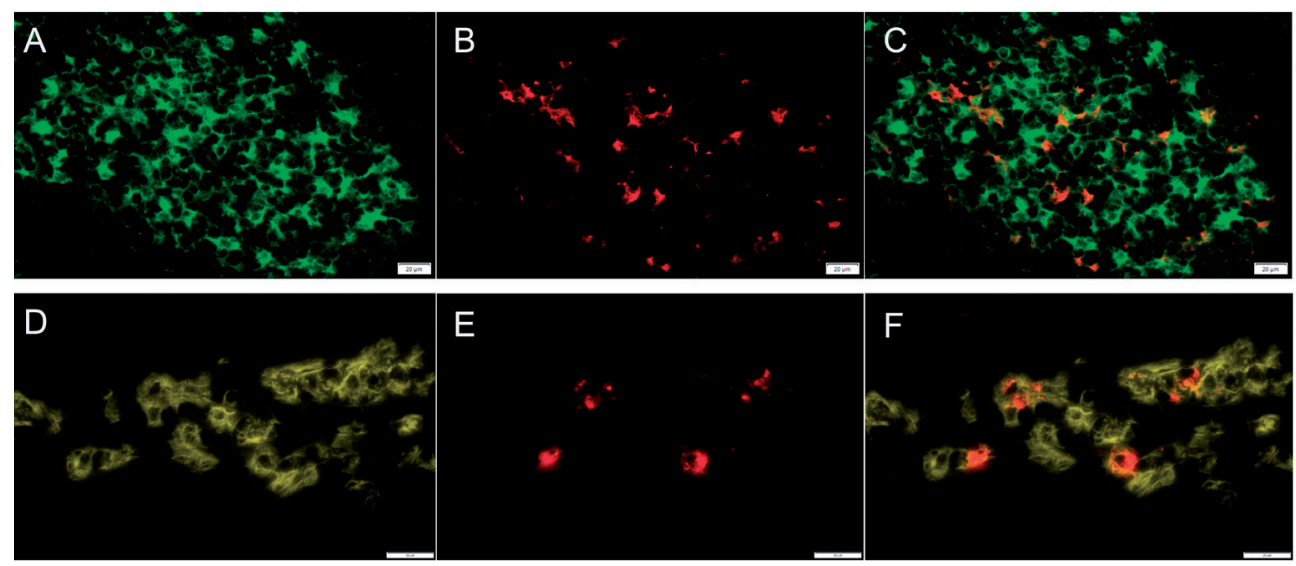

Fig 5. Co-localization of chlamydial antigen and follicular dendritic cells (A-C) or mononuclear phagocytes (D-F) in the caecal tonsil at 11 days post infection

A: Follicular dendritic cell staining with $m A b$ 74.3, B: Chlamydia staining with pAb LS-C85741, C: merge of $A$ and $B, D$ : mononuclear phagocytes staining with mAb KULO1, E: Chlamydia staining with $p A b$ LSC85741, F: merge of $D$ and $E$.

\section{Dissemination to other organs}

In the second and third experiment dissemination of Chlamydia to tissues outside the gastro-intestinal tract was investigated (Fig. S2). In both experiments no chlamydial DNA was detected in spleen samples. In experiment 3 at day 4, one kidney sample had a Cq value of 25 and one liver sample a Cq value of 39 (Fig. S2 B, red encircled). This was most probably a result of contamination, because a part of the gut ruptured during necropsy. Chlamydial DNA could only be detected scarcely in airsac, liver and lung with Cq values above 30 , and the presence of chlamydia antigen in these tissues could not be confirmed with IHC.

\section{Serologic response}

The ELISA results indicate the development of a serologic response against Chlamydia in all three experiments (Fig. S3). At day zero all serum samples had a corrected OD value below 0.3. From day seven or eight an increase in OD (450 nm) was observed in all experiments. The level of response varied between animals but each individual animal displayed increased antibody response in the course of infection. 


\section{Discussion}

C. gallinacea is a relatively recently discovered and widespread pathogen in poultry, but studies investigating the pathogenicity of $C$. gallinacea are still limited. Here, the pathogenicity of C. gallinacea strain NL_G47 was investigated in six-week old SPF layers. The layers were orally inoculated which resulted in throat- and cloacal shedding and infection of epithelial cells of the jejunum, ileum and caecum without signs of clinical disease, and macroscopic or histologic signs of inflammation. At day 11, chlamydial antigen was co-localised within macrophages in the lamina propria and FDCs in the caecal tonsil and, from day 7 onwards, a rise in antibody titre was shown. The presence of chlamydial antigen in epithelial cells of the gut, macrophages in the lamina propria and FDCs in the caecal tonsil, in combination with the development of an antibody response, has not been shown before for C. gallinacea.

Examination of the gut showed that the chlamydial load increased over time and towards the more distal parts, i.e. jejunum, ileum, caecum and colon, based on the results of qPCR. In the epithelial cells of the jejunum, ileum and caecum, the presence of chlamydial antigen was confirmed with immunohistochemistry. In contrast, the presence of chlamydial antigen in the epithelial cells of the colon could not be confirmed, although $\mathrm{Cq}$ values in the PCR overlapped at day 4, 8 and 11 in the jejunum, ileum, caecum and colon (see Fig.. 3). Reisolation of viable C. gallinacea from these tissues was not performed, because it would be very difficult to discriminate whether bacteria were present in epithelium or faecal content. However, the absence of chlamydial antigen in the colon epithelium suggests chlamydiae were only present in the lumen (residing in the fecal content) of the colon and replication in epithelial cells occured in the more proximal parts of the gut, i.e. jejunum, ileum and caecum.

Studies investigating the infection of Chlamydia in different parts of the poultry gut are limited. Experimental studies with $C$. psittaci in chickens reported the presence in the jejunum (12) or recovery of viable bacteria from the colo-rectum (13), but did not mention the presence of chlamydial antigen in the epithelium of the colon. In oral infections with C. psittaci in ducks, chlamydial antigen was detected in the caecum, but no data were presented about the colon (14). Therefore, we cannot conclude if this difference has been observed in other chlamydial infections in poultry as well.

Furthermore, the possible cause of the observed difference in chlamydial infection of the epithelium in the jejunum, ileum and caecum and colon, is unknown. Perhaps that differences in the microbiome or mucin layer related to the function of the various parts of the gut might facilitate or prevent epithelial infection. For example in mice and humans the epithelium of the colon mainly secretes peptides that bind and aggregate bacteria, while 
the ileum mainly produces antibacterial peptides that kill bacteria reaching proximity to the epithelium (15). Aggregation of bacteria could be a more successful barrier for chlamydial infection than killing by antimicrobial peptides. Further research into the role of the microbiome and mucin layer in chlamydial infection would help to understand how C. gallinacea infects the gut epithelium.

The asymptomatic presence of Chlamydia in the gut is regarded as a typical feature of Chlamydiae and has been described in virtually all hosts (16). In a murine model with C. muridarum, oral infection resulted in an adaptive immune response, but infections in the caecum were not resolved and did lead to pathologic changes, probably due to the downregulation of the local immune response (17). These findings are in line with the results of our study. We did measure an increase in antibody response from day 7 onward, which might be an underestimation of the response against $C$. gallinacea as a mix of $C$. abortus and $C$. trachomatis antigen was used. Furthermore, we did not observe macroscopic or histological signs of inflammation in the gut, although chlamydial antigen was present in jejunum, ileum and caecum. We could also co-localise chlamydial antigen within macrophages in the lamina propria and FDCs in the caecal tonsil, which probably reflects the successful probing or uptake of chlamydiae by macrophages / dendritic cells from the intestinal lumen and subsequent presentation of antigen to FDCs in the B cell follicles resulting in the increase of the adaptive immune response.

A successful adaptive (systemic) immune response could also explain the limited systemic dissemination of $C$. gallinacea and might be a consequence of its relatively nonpathogenic nature in chickens. In our study chlamydial DNA was only detected incidently in airsac, liver and lung and the presence of chlamydial antigen was not confirmed with IHC. Reisolation of viable Chlamydia was not performed, but the limited systemic dissemination of $C$. gallinacea is in line with findings in other studies $(4,5,7)$. Studies investigating the pathogenic potential of $C$. abortus and $C$. psittaci in comparative chicken models, revealed expression of both immunologically relevant and bacterial relevant factors was higher in C. psittaci infection $(18,19)$. These differences could explain why C. psittaci is more invasive than C. abortus in avian hosts. It would be useful to perform similar studies with C. gallinacea to further understand its pathogenic nature and hostpathogen interaction. In particular, because chickens are considered the natural host of $C$. gallinacea (4) and in contrast to C. abortus for which small ruminants are considered the predominant host (20).

Although our study focused on the short term health effects, C. gallinacea could cause persistent infections in gut epithelium due to the possible local downregulation of the immune response as hypothesised earlier. In our experiments, C. gallinacea was still highly present at the end of the experiments at day 11 . In other studies $C$. gallinacea was 
detected in the rectum at day 26 post infection (5), or for at least three months in cloacal swabs (4) suggesting a persistent infection of the gut. Persistent infections of Chlamydia in the gut can result in long term or chronic health effects (21), because an infection in gut epithelial cells (due to a possible higher cell turnover) and an increase in adaptive immune response will result in (metabolic) costs that might have an adverse effect on production parameters (22). A negative effect of C. gallinacea infection on production has already been shown in broilers with reduced weight (4). In layers, this effect, on for example egg production, deserves further investigation. Though, this type of field research would require a rigorous design considering the high prevalence of C. gallinacea at farm level (2).

In conclusion, our study confirms C. gallinacea infection mainly resides in the gut and results in asymptomatic cloacal shedding. The combination of asymptomatic shedding and possible persistent infection of the gut could result in adverse long term health effects. Furthermore, persistent cloacal shedding of $C$. gallinacea facilitates orofecal transmission and probably explains why it is highly endemic in poultry $(2,4,23)$.

\section{Funding}

This work was supported by the Dutch Ministry of Agriculture, Nature and the Environment (grant WOT-01-002-005.02, WOT-01-01-002-005.13 and KB-21-006-022).

\section{Acknowledgements}

The authors acknowledge the animal care takers of WBVR for their assistance during the animal experiments; Sebastiaan van der Broek, Lars Ravesloot and Rob Zwart of WBVR for preparing the samples for immunohistochemistry; Eugenie Ellen, Irene Oud and Marianne Vahl of WBVR for their technical assistance in the PCR analyses of the samples; Marielle den Esker of WBVR for proofreading. 


\section{Supporting information}

S1 Table. Scoring card clinical signs

\begin{tabular}{|c|c|c|c|}
\hline & No signs (0) & Mild (1) & Severe (2) \\
\hline Mental state & $\begin{array}{l}\text { Active, makes noise, } \\
\text { responds to environment } \\
\text { or handling }\end{array}$ & $\begin{array}{l}\text { Less active, bulging } \\
\text { (with feathers upright), } \\
\text { but responding to } \\
\text { environment }\end{array}$ & $\begin{array}{l}\text { No response to } \\
\text { environment, lying, } \\
\text { retreating, hardly to no } \\
\text { response to handling, } \\
\text { stopped eating and } \\
\text { drinking }\end{array}$ \\
\hline Head & $\begin{array}{l}\text { No discharge from nose } \\
\text { or eye, no red eyes }\end{array}$ & $\begin{array}{c}\text { Watery to mucous } \\
\text { discharge from eye and/ } \\
\text { or nose (tear stripe), red } \\
\text { eyes }\end{array}$ & $\begin{array}{l}\text { Severe mucous or bloody } \\
\text { discharge and/or dense } \\
\text { red, swollen eyes }\end{array}$ \\
\hline Upper airways & $\begin{array}{c}\text { No sneezing or shaking } \\
\text { with the head }\end{array}$ & Occasionally sneezing & $\begin{array}{l}\text { Frequent sneezing and } \\
\text { shaking of the head }\end{array}$ \\
\hline Lower airways & $\begin{array}{l}\text { No increased respiration } \\
\text { frequency or symptoms } \\
\text { of shortness of breath }\end{array}$ & $\begin{array}{l}\text { Slightly increased } \\
\text { respiration frequency } \\
\text { and / or noises such as } \\
\text { gargling and grating }\end{array}$ & $\begin{array}{c}\text { Clearly increased } \\
\text { breathing frequency, } \\
\text { open mouth, stretched } \\
\text { neck, symptoms of } \\
\text { shortness of breath, } \\
\text { noises such as gargling } \\
\text { and rattling }\end{array}$ \\
\hline $\begin{array}{l}\text { Gait and } \\
\text { balance }\end{array}$ & $\begin{array}{c}\text { Normal gait, no } \\
\text { uncoordinated } \\
\text { movements or tremors }\end{array}$ & $\begin{array}{c}\text { Difficulty with } \\
\text { coordination when } \\
\text { standing up, can } \\
\text { walk but seems to } \\
\text { have more difficulty } \\
\text { with coordination of } \\
\text { movements } \\
\end{array}$ & $\begin{array}{c}\text { Disturbed balance, } \\
\text { difficulty walking or } \\
\text { paralysis, twisted neck, } \\
\text { walking in circles, severe } \\
\text { muscle tremors }\end{array}$ \\
\hline Feces & $\begin{array}{l}\text { Normal chicken feces, no } \\
\text { abnormal consistency or } \\
\text { color }\end{array}$ & $\begin{array}{l}\text { Feces with abnormal color } \\
\text { (green to yellow) and/or } \\
\text { consistency (wetter) }\end{array}$ & $\begin{array}{c}\text { Feces with abnormal color, } \\
\text { consistency and quantity, } \\
\text { presence of blood }\end{array}$ \\
\hline
\end{tabular}


Experiment 2

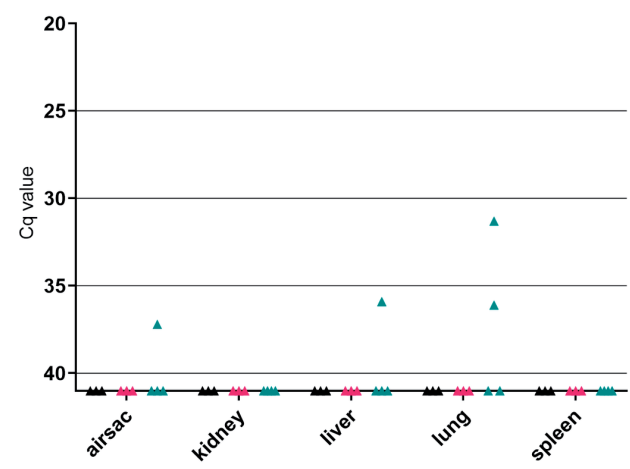

- Day 4 A Day 8 \& Day 11
Experiment 3



$\triangle$ Day 4 Day 8 Day 11

S2 Fig. PCR results of tissue samples outside the gastro-intestinal tract

$A$ shows the results of experiment 2 and $B$ of experiment 3. Per timepoint the results of the individual samples are shown. The results of day 0 are not presented. On the $Y$-axis the cycle treshold ( $\mathrm{Cq}$ ) value is depicted. The axis has been rotated in order to make the graph more intuitive. Red circles indicate falsepositives (see text).
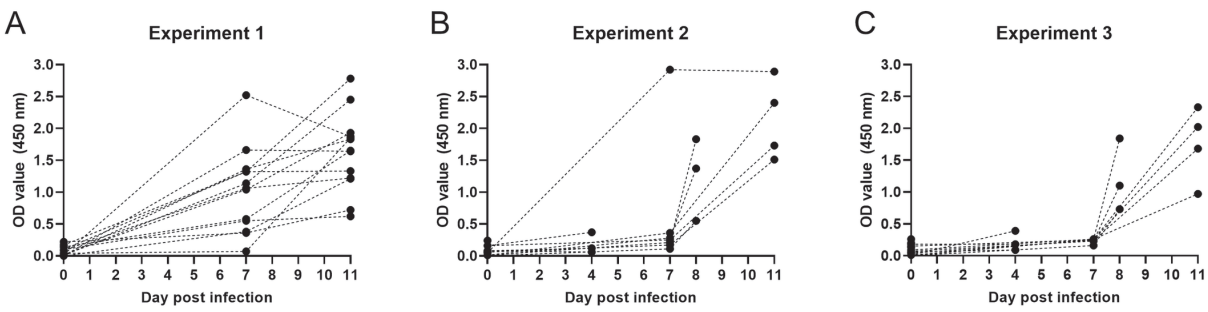

\section{S3 Fig. ELISA results of experiment 1,2 and 3}

In $B$ and $C$ the number of animals decreases due to sequential sacrificing. Every dot represents a sampling moment. 


\section{References}

1. Sachse K, Laroucau K, Riege K, Wehner S, Dilcher M, Creasy HH, et al. Evidence for the existence of two new members of the family Chlamydiaceae and proposal of Chlamydia avium sp. nov. and Chlamydia gallinacea sp. nov. Systematic and applied microbiology. 2014;37(2):79-88.

2. Heijne M, van der Goot JA, Fijten H, van der Giessen JW, Kuijt E, Maassen CBM, et al. A cross sectional study on Dutch layer farms to investigate the prevalence and potential risk factors for different Chlamydia species. PloS one. 2018;13(1):e0190774.

3. Ornelas-Eusebio E, Garcia-Espinosa G, Vorimore F, Aaziz R, Durand B, Laroucau K, et al. Crosssectional study on Chlamydiaceae prevalence and associated risk factors on commercial and backyard poultry farms in Mexico. Preventive veterinary medicine. 2020;176:104922.

4. Guo W, Li J, Kaltenboeck B, Gong J, Fan W, Wang C. Chlamydia gallinacea, not C. psittaci, is the endemic chlamydial species in chicken (Gallus gallus). Scientific reports. 2016;6:19638.

5. You J, Wu Y, Zhang X, Wang X, Gong J, Zhao Z, et al. Efficient fecal-oral and possible vertical, but not respiratory, transmission of emerging Chlamydia gallinacea in broilers. Veterinary microbiology. 2019;230:90-4.

6. Heijne M, Jelocnik M, Umanets A, Brouwer MSM, Dinkla A, Harders F, et al. Genetic and phenotypic analysis of the pathogenic potential of two novel Chlamydia gallinacea strains compared to Chlamydia psittaci. Scientific reports. 2021;11(1):16516.

7. Laroucau K, Vorimore F, Aaziz R, Berndt A, Schubert E, Sachse K. Isolation of a new chlamydial agent from infected domestic poultry coincided with cases of atypical pneumonia among slaughterhouse workers in France. Infection, genetics and evolution : journal of molecular epidemiology and evolutionary genetics in infectious diseases. 2009;9(6):1240-7.

8. Guo W, Jelocnik M, Li J, Sachse K, Polkinghorne A, Pannekoek Y, et al. From genomes to genotypes: molecular epidemiological analysis of Chlamydia gallinacea reveals a high level of genetic diversity for this newly emerging chlamydial pathogen. BMC genomics. 2017;18(1):949.

9. Holzer M, Barf LM, Lamkiewicz K, Vorimore F, Lataretu M, Favaroni A, et al. Comparative Genome Analysis of 33 Chlamydia Strains Reveals Characteristic Features of Chlamydia Psittaci and Closely Related Species. Pathogens. 2020;9(11).

10. Kik M, Heijne M, ljzer J, Grinwis G, Pannekoek Y, Gröne A. Fatal <em>Chlamydia avium</em> Infection in Captive Picazuro Pigeons, the Netherlands. Emerging Infectious Disease journal. 2020;26(10):2520.

11. Ehricht R, Slickers $P$, Goellner $S$, Hotzel H, Sachse K. Optimized DNA microarray assay allows detection and genotyping of single PCR-amplifiable target copies. Molecular and cellular probes. 2006;20(1):60-3.

12. Yin L, Kalmar ID, Lagae $S$, Vandendriessche $S$, Vanderhaeghen W, Butaye $P$, et al. Emerging Chlamydia psittaci infections in the chicken industry and pathology of Chlamydia psittaci genotype $B$ and $D$ strains in specific pathogen free chickens. Veterinary microbiology. 2013;162(2-4):740-9. 
13. Takahashi T, Takashima I, Hashimoto N. Shedding and transmission of Chlamydia psittaci in experimentally infected chickens. Avian diseases. 1988;32(4):650-8.

14. Thierry S, Vorimore F, Rossignol C, Scharf S, Sachse K, Berthon P, et al. Oral Uptake of Chlamydia psittaci by Ducklings Results in Systemic Dissemination. PloS one. 2016;11(5):e0154860.

15. Schroeder BO. Fight them or feed them: how the intestinal mucus layer manages the gut microbiota. Gastroenterol Rep (Oxf). 2019;7(1):3-12.

16. Rank RG, Yeruva L. Hidden in plain sight: chlamydial gastrointestinal infection and its relevance to persistence in human genital infection. Infection and immunity. 2014;82(4):1362-71.

17. Yeruva L, Spencer N, Bowlin AK, Wang Y, Rank RG. Chlamydial infection of the gastrointestinal tract: a reservoir for persistent infection. Pathogens and disease. 2013;68(3):88-95.

18. Braukmann M, Sachse K, Jacobsen ID, Westermann M, Menge C, Saluz HP, et al. Distinct intensity of host-pathogen interactions in Chlamydia psittaci- and Chlamydia abortus-infected chicken embryos. Infection and immunity. 2012;80(9):2976-88.

19. Kalmar I, Berndt A, Yin L, Chiers K, Sachse K, Vanrompay D. Host-pathogen interactions in specific pathogen-free chickens following aerogenous infection with Chlamydia psittaci and Chlamydia abortus. Veterinary immunology and immunopathology. 2015;164(1-2):30-9.

20. Essig A, Longbottom D. Chlamydia abortus: new aspects of infectious abortion in sheep and potential risk for pregnant women. Current clinical microbiology reports. 2015;2(1):22-34.

21. Reinhold P, Sachse K, Kaltenboeck B. Chlamydiaceae in cattle: commensals, trigger organisms, or pathogens? Veterinary journal. 2011;189(3):257-67.

22. Kogut $\mathrm{MH}$, Genovese $\mathrm{KJ}$, Swaggerty $\mathrm{CL}$, He H, Broom L. Inflammatory phenotypes in the intestine of poultry: not all inflammation is created equal. Poultry science. 2018;97(7):2339-46.

23. Hulin V, Oger S, Vorimore F, Aaziz R, de Barbeyrac B, Berruchon J, et al. Host preference and zoonotic potential of Chlamydia psittaci and C. gallinacea in poultry. Pathogens and disease. 2015;73(1):1-11. 

CHAPTER 5 


\section{Host-pathogen interactions during experimental cross infection of Chlamydia gallinacea and Chlamydia psittaci in chickens}

Marloes Heijne ${ }^{1 *}$, Jeanet van der Goot², Herma Buys², Annemieke Dinkla', Hendrik Jan Roest $^{1,3}$, Lucien van Keulen', Ad Koets ${ }^{1,4}$

\footnotetext{
${ }^{1}$ Department of Bacteriology, Host-Pathogen Interaction and Diagnostics Development, Wageningen Bioveterinary Research, Lelystad, the Netherlands

${ }^{2}$ Department of Diagnostics and Crisis Organisation, Wageningen Bioveterinary Research, Lelystad, the Netherlands

${ }^{3}$ Current affiliation: Directorate Animal Supply Chain and Animal Welfare, Ministry of Agriculture, Nature and Food Quality, The Hague, the Netherlands

${ }^{4}$ Department of Population Health Sciences, Faculty of Veterinary Medicine, Utrecht University, Utrecht, the Netherlands
} 


\section{Abstract}

Chlamydia psittaci was considered the predominant chlamydial species in poultry until Chlamydia gallinacea was discovered in 2009. C. psittaci is a zoonotic obligate intracellular bacterium reported in more than 465 bird species including poultry. In poultry, infections can result in asymptomatic disease, but also in more severe systemic illness. The zoonotic potential of $C$. gallinacea has yet to be proven. Infections in poultry appear to be asymptomatic and in recent prevalence studies $C$. gallinacea was the main chlamydial species found in chickens. The high prevalence of C. gallinacea resulted in the question if an infection with C. gallinacea might protect against an infection with C. psittaci.

To investigate possible cross protection, chickens were inoculated with C. gallinacea NL_G47 and subsequently inoculated with either a different strain of C. gallinacea (NL_F725) or C. psittaci. Chickens that had not been pre-inoculated with C. gallinacea NL_G47 were used as a C. gallinacea or C. psittaci infection control. In the groups that were inoculated with C. psittaci, no difference in throat or cloacal shedding, or in tissue dissemination was observed between the control group and the preinoculated group. In the groups inoculated with C. gallinacea NL_F725, shedding in cloacal swabs and tissues dissemination was lower in the group pre-inoculated with C. gallinacea NL_G47.

These results indicate previous exposure to $C$. gallinacea does not protect against an infection with $C$. psittaci, but might protect against a new infection of $C$. gallinacea. 


\section{Introduction}

Chlamydia gallinacea and Chlamydia psittaci belong to the Chlamydiaceae, a family of obligate intracellular bacteria that currently exists of one genus and 14 species (1). Until 2009, C. psittaci was considered the predominant chlamydial species in poultry. C. psittaci is ubiquitous and has been reported in several mammalian species and more than 465 bird species including poultry (2). Infections can remain asymptomatic, but also result in severe systemic illness and mortality depending on the chlamydial strain, host, host age and (environmental) stressors (3). Moreover, C. psittaci has a known zoonotic potential; infections in humans can ultimately result in severe pneumonia (3).

In 2009, a novel Chlamydia species was identified in poultry and later classified as C. gallinacea $(4,5)$. Soon after its discovery, it became clear that $C$. gallinacea is highly prevalent in chickens (6-8). Infections with C. gallinacea do not result in clinical signs of disease, but can lead to production loss such as reduced weight gain (8). There is currently no microbiological evidence of a zoonotic potential of $C$. gallinacea, although $C$. gallinacea has been considered the causative agent in cases of pneumonia in slaughterhouse workers $(5,6)$.

In a Dutch cross sectional study in 2018, C. gallinacea was detected by PCR in pooled faecal samples at 71 of the 151 investigated layer farms. C. psittaci was not detected in any sample from these farms (6). This was unexpected, since a Belgian study in 2014 reported 6/7 broiler breeder, 7/7 broiler and 5/5 layer farms PCR and culture positive for $C$. psittaci in pharyngeal swabs (9). Other studies in Belgium and Northern-France from 2010 and 2013 also reported a high prevalence of $C$. psittaci determined with $P C R$, culture (on pharyngeal swabs and tissues) and/or serology $(10,11)$. Culture with PCR confirmation is Chlamydia species specific, but with the current understanding of $C$. gallinacea in poultry, a high seroprevalence might also be explained by possible cross reactive antibodies as a major outer membrane protein (MOMP) based C. psittaci ELISA was used (11).

Cross reactive antibodies between chlamydial species are known to occur because of the close structural similarity among some of the major surface antigens such as MOMP (12) and, could potentially result in cross protection against multiple Chlamydia species. Apart from differences in methodology, this may offer an explanation why C. psittaci was not detected in the Dutch prevalence study (6) or, vice versa, C. gallinacea was not detected in the Belgian prevalence study (9). We therefore hypothesised that the high prevalence of C. gallinacea in Dutch layers resulted in herd immunity against $C$. psittaci due to possible cross protection. 
To investigate the hypothesis of possible cross protection, chickens were inoculated with C. gallinacea NL_G47 and, after five weeks, inoculated with either a different strain of $C$. gallinacea (NL_F725) or with a strain of C. psittaci. These treatments were compared to single exposure with either C. gallinacea (NL_F725) or C. psittaci. Reduced shedding or tissue dissemination in the groups that had been pre-inoculated with C. gallinacea $\mathrm{NL}_{-}$ G47 would be an indication of possible cross protection between C. gallinacea strains and/ or C. psittaci. Cross protection between C. gallinacea and C. psittaci could be a beneficial scenario from a one health perspective, because infections with $C$. gallinacea seem relatively harmless for poultry and C. gallinacea has no proven zoonotic potential thus far.

\section{Materials and methods}

\section{Ethical statement}

The animal experiment was conducted in accordance with the national regulations on animal experimentation. The project was approved by the Dutch Central Authority for Scientific Procedures on Animals (CCD) (permit number AVD4010020173926).

\section{Inocula}

Chlamydia gallinacea NL_G47 and NL_F725 were isolated from caecal material from laying hens as described earlier (13). Chlamydia psittaci strain NL_Borg is an in-house reference strain closely related to the turkey outbreak strain C. psittaci NJ1 (13). All strains were passaged three times in the yolk sac of embryonated SPF chicken eggs and stored at $-80^{\circ} \mathrm{C}$ in a $20 \%$ yolk sac suspension in Sucrose Phosphate Glutamate (SPG) until inoculation (14). The infectious dose of the suspensions was calculated via egg titration experiments and expressed as the Egg Infectious Dose $50\left(\operatorname{EID}_{50}\right)(13,15,16)$.

\section{Animals and housing}

A total of 48 five-week-old Specified Pathogen Free (SPF) White Leghorn layers were obtained from Royal GD (Deventer, the Netherlands). Chlamdiaceae are not included in standard SPF testing, therefore 10 cloacal swabs from layers of the mother flock were collected. All swabs tested PCR negative for Chlamydia spp. before the chickens were delivered. All chickens had a seven-day acclimatization period prior to the first inoculation.

After arrival the hens were housed in groups on sawdust bedding in temperaturecontrolled rooms under optimal light conditions and humidity. Feed and water were provided ad libitum. Control chickens or chickens infected with C. gallinacea were housed in veterinary biosafety level 2 (vBSL 2) facilities and chickens infected with C. psittaci were housed in biosafety level 3 (BSL 3) facilities at Wageningen Bioveterinary Research (WBVR, Lelystad, the Netherlands). 


\section{Experimental design}

The experiment consisted of two parts as shown in Fig. 1A. In the first part 26 randomly selected chickens were orally inoculated with C. gallinacea NL_G47 seven days after arrival. The remaining twenty-two chickens were not inoculated and served as a control group. Both groups were housed seperately and chickens were numbered randomly. At day 28, after the first inoculation, the groups were transported to a new location with BSL-3 facilities. Both groups were transported separately to prevent cross contamination.

At the new location the control group and the infected group were further subdivided in two groups (resulting in four experimental groups, Fig. 1A). The chickens were allocated to the groups alternately by number. At day 35, chickens were either inoculated with $C$. gallinacea strain NL_F725 or with the C.psittaci NL_Borg strain. For C.gallinacea inoculation was performed orally, because the faecal-oral route is the main route of transmission (17). For C. psittaci inoculation was performed oro-nasally, because both the respiratory and oral route have been described (18). At day 42 the animals were sacrificed (see Fig. 1B).

The inoculations were performed with a $1 \mathrm{ml}$ syringe (Terumo Europe N.V.) and an oral gavage needle (18Gx1,5", Terumo Europe N.V.). For the oro-nasal inoculation, chickens first received one droplet of the suspension in one nostril after which the remaining suspension was inoculated orally. At the first inoculation with C. gallinacea NL_G47 chickens received $0.5 \mathrm{ml}$ of a yolk suspension of NL_G47 with an infectious dose of $10^{5.9}$ EID $_{50}$ per ml. At the second round chickens were inoculated with either $0.5 \mathrm{ml}$ of a yolk suspension of NL_F725 or with $0.5 \mathrm{ml}$ of a yolk suspension of $C$. psittaci NL_Borg, both with an infectious dose of $10^{5.4}$ EID $_{50}$ per $\mathrm{ml}$. The inoculation dose was confirmed by back-titration and the infectious dose was within a range of one log step of the initial dose.

In the first part of the experiment the weight of the animals was recorded at day 0 (before inoculation) and day 28. During the whole experiment (42 days) clinical signs were recorded daily according to a clinical scoring card (Additional file 1). In the first part of the experiment, cloacal swabs (Puritan HydraFlock sterile swab, ITK Diagnostics BV) were collected at day $0,4,7,14$ and 28 . In the second part cloacal swabs were collected daily from day 35 until day 42 in all groups. In the C. psittaci exposed groups, additional throat swabs were collected daily, because for $C$. psittaci throat swabs might be a more sensitive method to measure shedding (19). In the C. gallinacea groups no throat swabs were collected based on earlier results that showed shedding in cloacal swabs was higher (14). In the first part serum samples were collected at day 0 , day 7, day 14, day 28 and, in the second part, at day 35 and day 42 at necropsy. All samples at day 0 were collected prior to inoculation to confirm the absence of a current Chlamydia infection. A timeline of the experiments including sampling moments is given in Fig. 1B. 
At the end of the experiment the chickens were euthanized by maximum blood collection via heart puncture, under anesthesia by intramuscular injection of a mixture of $0.3 \mathrm{ml} /$ kg ketamine (Ketamine 10\%, Alfasan Diergeneesmiddelen B.V.) and $0.5 \mathrm{ml} / \mathrm{kg}$ xylazine (Sedamun, Dechra Veterinary Products).

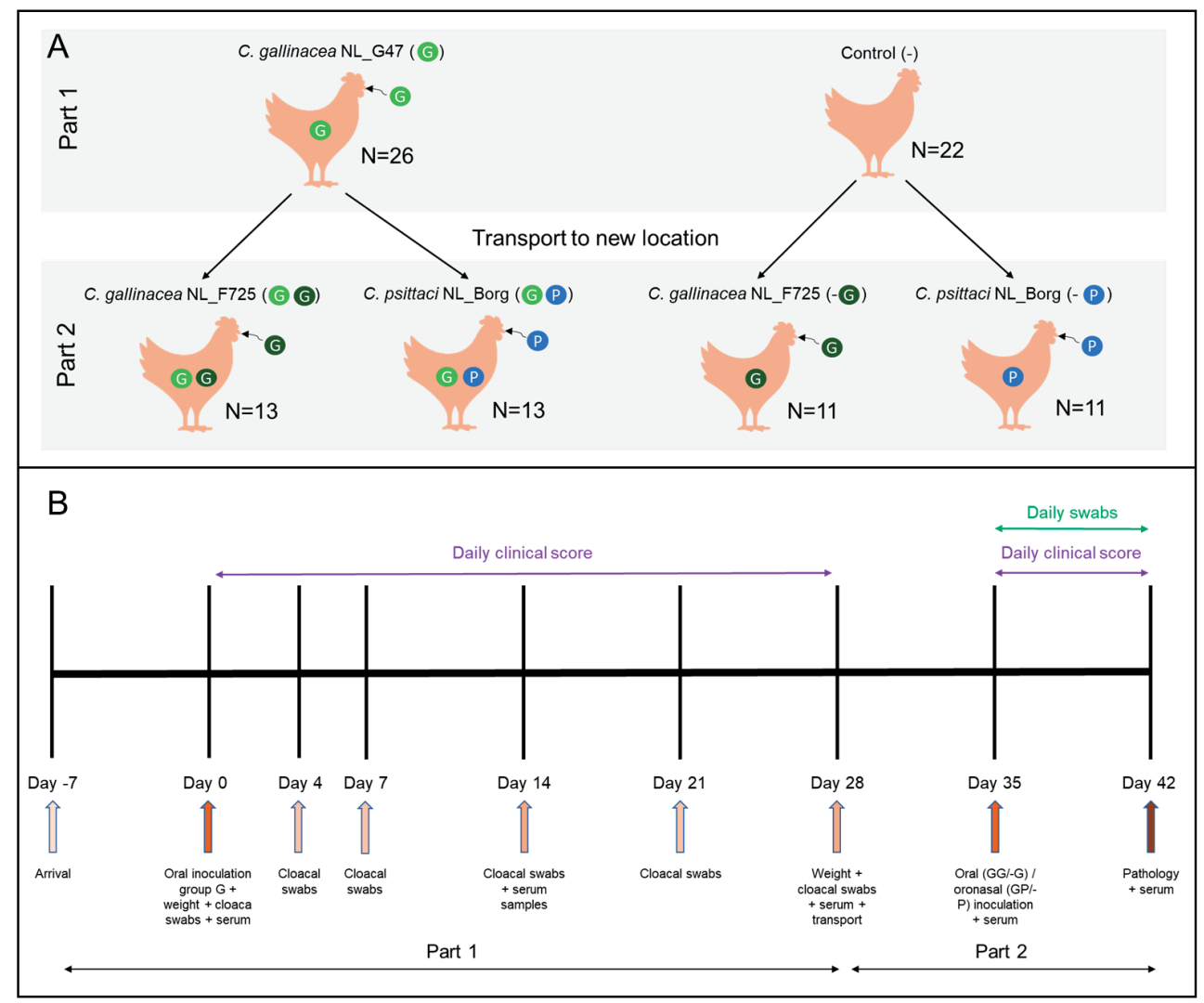

Fig. 1. Experimental design

A shows the experimental setup with group size and type of inoculum (coloured bacteria) and $B$ the timeline of the experiment with sampling moments. From day 35 to day 42 daily throat and cloacal swabs were collected in the C. psittaci inoculated group GP and -P. In group GG and -G only daily cloacal swabs were collected.

\section{Necropsy}

At necropsy carcasses were opened on a clean plastic sheet which was replaced after each necropsy. To prevent cross contamination new sterile instruments and petridishes were used for every tissue sample. Tissue samples (approximately $0.5 \mathrm{~cm}^{3}$ ) were collected from the airsac, lung, liver, spleen, kidney, jejunum, ileum, caecum, caecal tonsil and colon. Samples for PCR were collected in $1 \mathrm{ml}$ SPG in Lysing Matrix D tubes (MP Biomedicals) and ribolysed ( $2 \times 20 \mathrm{sec}$ at $4 \mathrm{~m} / \mathrm{sec}$ ) before storage at $-80^{\circ} \mathrm{C}$. Tissue samples for histology and 
immunohistochemistry were collected in $10 \%$ neutral buffered formalin and routinely processed into paraffin blocks.

\section{PCR analyses}

Swabs were immersed in $1.5 \mathrm{ml}$ PBS 13 (BM014, WBVR) and thoroughly vortexed (10s) to suspend the sample from the swab. From the swab or tissue suspension, $200 \mu$ was used for DNA extraction. Swabs and tissues suspensions from the BSL-3 lab were heated for 30 minutes at $99^{\circ} \mathrm{C}$ before cell lysis to prevent transfer of infectious material from the containment area. In a prior pilot experiment was established that the heating step did not influence the PCR outcome. DNA extraction was performed with a MagNA Pure LC total Nucleic Acid Isolation kit in the MagNA Pure ${ }^{\circledast}$ system (Roche Diagnostics) according to instructions provided by the manufacturer. All DNA samples were tested with a Chlamydiaceae PCR targeting the 23S rRNA as has been previously described in detail (6, 20). Samples from chickens that were exposed to $C$. psittaci were also tested with a specific C. psittaci PCR targeting the ompA gene according to methods published previously (21).

\section{Histology and immunohistochemistry}

Formalin fixed tissue samples were cut into $4 \mu \mathrm{m}$ sections and collected on positively charged glass slides (SuperfrostPlus ${ }^{\circledR}$, Thermo Scientific). Sections were then stained with haematoxylin-eosin (HE) or immunostained with a polyclonal anti-Chlamydia antibody (LS-C85741) or a monoclonal anti-Chlamydia antibody (MBS830551). Epitope retrieval of the formalin fixed sections consisted of proteolysis induced epitope retrieval for the polyclonal antibody $\left(0,1 \%\right.$ protK in TBS for $30 \mathrm{~min}$ at $\left.37^{\circ} \mathrm{C}\right)$ and heat induced epitope retrieval (citrate buffer, $\mathrm{pH} 6.0,121^{\circ} \mathrm{C}$ for $5 \mathrm{~min}$ ) for the monoclonal antibody. Anti-rabbit or anti-mouse HRP conjugated polymer was used as a secondary antibody (Invitrogen) and $\mathrm{DAB}+$ as substrate (Dako, Agilent). Sections were counterstained with Mayer's hematoxylin and mounted permanently. Photographs were taken with an Olympus BX51 microscope equipped with a high-resolution digital camera.

\section{Serology}

Serum samples were tested with an in-house ELISA using a commercially available mix of Chlamydia abortus and Chlamydia trachomatis antigens (Institut Virion\Serion $\mathrm{GmbH}$ ), because specific serological tests for $C$. gallinacea are currently not commercially available. An antigen coating solution was prepared with a final concentration of $4 \mu \mathrm{g} /$ $\mathrm{ml}$ of each antigen in bicarbonate coating buffer with pH 9.6 (BM112, WBVR,). Ninety-sixwell microtiter plates (Nunc MaxiSorp ${ }^{\mathrm{TM}}$, Thermo Fisher Scientific) were coated overnight at $37^{\circ} \mathrm{C}$ with $100 \mu \mathrm{l}$ per well in coating buffer. Following six washes with $0.05 \%$ Tween $^{\circledR} 80$, the plates were blocked with $190 \mu \mathrm{l}$ per well of $5 \%$ skimmed-milk powder (Elk, Campina) in Tris-buffered saline with $0.1 \%$ Tween $^{\circ} 20$ detergent (TBST, BM309, WBVR) for 60 min at room temperature (RT). The plates were washed as described above, subsequently 
$100 \mu \mathrm{l}$ of chicken serum per well (diluted 1:500 in 5\% skimmed milk powder-TBST) was added and the plates were incubated for $60 \mathrm{~min}$ at $37^{\circ} \mathrm{C}$. After further washing, $100 \mu \mathrm{l}$ of goat anti-chicken $\lg \mathrm{Y}(\mathrm{H}+\mathrm{L})-\mathrm{HRP}$ (Southern Biotech), diluted 1:6,000 in 5\% skimmed milk powder-TBST) was added per well, and the mixture was incubated for $60 \mathrm{~min}$ at $37^{\circ} \mathrm{C}$. Again six washes with $0.05 \%$ Tween $^{\circledR} 80$ were performed and one wash with Super- $\mathrm{Q}^{\circledast}$ water. Bound antibody was detected with TMB One component HRP Microwell substrate (TMBW-1000-01, SurModics). The reaction was terminated after $10 \mathrm{~min}$ by the addition of $100 \mu \mathrm{l} 0.5 \mathrm{M}$ sulfuric acid. The optical density (OD) was measured at $450 \mathrm{~nm}$ on a Thermo Labsystems Multiskan RC microplate reader (Thermo Fisher Scientific).

Per plate, two plate controls were included with two wells per control. In one control, no serum and no conjugate was added to the wells, in the other control no serum was added. All obtained chicken sera were tested in one batch and the individual OD values were corrected for plate differences by subtracting the mean OD value of the plate control (without serum but with conjugate).

\section{Statistics}

Groups were compared using a linear mixed model with $\mathrm{Ct}$ value as outcome, for the swabs Day and Group were fixed effects and Chicken a random effect. For the model with the organs, Organ and Group were fixed effects and Chicken a random effect. Models with and without Group were compared by the likelihood ratio test. Analyses were performed in $\mathrm{R}(22)$, using the package Ime4.

\section{Results}

To investigate possible cross protection chickens were first inoculated with C. gallinacea NL_G47 (part 1 of the study) and after five weeks inoculated with C. gallinacea NL_F725 or C. psittaci NL_Borg (part 2 of the study). During part 1 the control group was not inoculated (see experimental design in Fig. 1).

\section{Part 1: primary inoculation with C. gallinacea NL_G47}

The group that was inoculated with C. gallinacea NL_G47 (group G) in part 1 of the experiment was successfully infected (Fig. 2A-C). All cloacal swabs tested positive in the Chlamydiaceae PCR at day 7 and after day 14 shedding declined as shown in Fig. 2A and B. Before transport at day 28, 19 / 26 cloacal swabs were PCR positive (i.e. $\mathrm{Ct}<40$ ) with a mean $\mathrm{Ct}$ of 35.6. Furthermore, a rise in antibody titre in the ELISA was observed (Fig. 2C). The uninfected control group (-) remained PCR negative in cloacal swabs and seronegative in the ELISA (Fig. 2A-C). During the first 28 days after inoculation no clinical signs, nor a difference in weight was observed in the controls and C. gallinacea inoculated chickens (Additional file 2). 


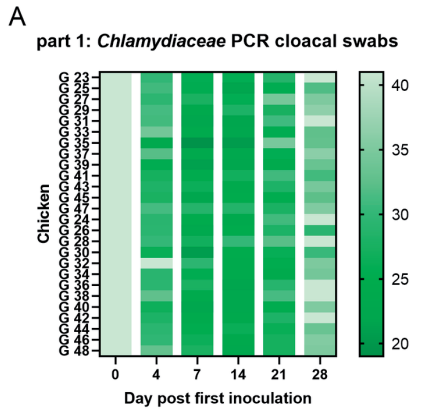

B

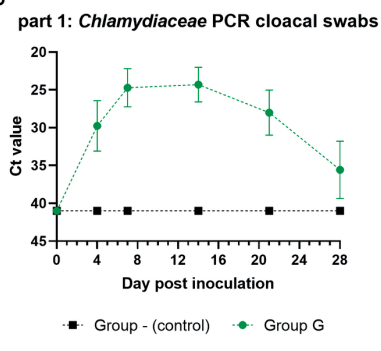

C

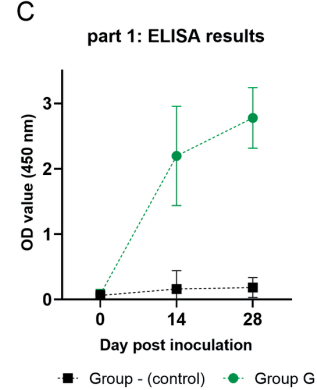

Fig. 2. PCR results of cloacal swabs ( $A$ and $B$ ) and ELISA results of serum samples (C)

In $A$ results of individual cloacal swabs of group $G$ in the Chlamydiaceae PCR per timepoint are depicted in a heatmap. The darker the colour, the lower the Ct value as shown in the colour scale at the right side. $C t$ values $>40$ or no PCR signal are shown as Ct 41. The results of the negative control group (-) are not shown. $B$ shows the mean $C t$ value of the cloacal swabs in time per group (of the Chlamydiaceae PCR). The error bar indicates the SD. On the $Y$-axis the cycle treshold (Ct) value is depicted. The Y-axis has been rotated and $C t$ values $>40$ or no $P C R$ signal are shown as $C t 41$. In $C$ the mean $O D(450 \mathrm{~nm})$ value of the serum samples per group per timepoint is shown. The error bar indicates the SD.

\section{Part 2: secondary inoculations}

In part 2 both groups were split resulting in four experimental groups. Two groups (GG and $-\mathrm{G}$ ) were inoculated with C. gallinacea NL_F725 and two groups (GP and -P) were inoculated with C. psittaci (Fig. 1A).

Secondary inoculation with C. psittaci (group GP and -P)

The C. psittaci inoculated groups (GP and -P) were tested with a Chlamydiaceae PCR and a specific $C$. psittaci PCR, which does not cross react with $C$. gallinacea. Before inoculation at day 35, 6/13 throat (mean Ct 37.9) and 10/13 cloacal swabs (mean Ct 34.6) of group GP test positive in the Chlamydiaceae PCR, but negative in the C. psittaci PCR (Fig. 3A and C, Additional file $3 A-B)$. This can be explained by the remaining presence of $C$. gallinacea NL_G47 and is in line with the findings at day 28 (Fig. 2A-B). From day 36 onwards, the mean $\mathrm{Ct}$ value in the Chlamydiaceae PCR in throat and cloacal swabs of group GP is lower than the mean Ct value in the C. psittaci PCR (Additional file 3A and B). This difference seems to be caused by the remaining presence of $C$. gallincea NL_G47 until day 37 in throat swabs and until day 38 in cloacal swabs, also when the results of group -P are taken into account. At the remaining days, a difference in sensitivity between both PCRs might also play a role (Additional file 3 ).

After inoculation, PCR based shedding of C. psittaci was higher in throat swabs as compared to cloacal swabs and based on the throat swabs, no significant difference between the groups was observed (Fig.. 3). At day 7, 11/13 throat swabs tested C. psittaci PCR positive in group GP as compared to 11/11 in group -P (Fig.. 3A). In cloacal swabs, 4 chickens tested C. psittaci PCR positive at day 7 in both groups (Fig. $3 C$ ). 
A Results C. psittaci PCR throat swabs

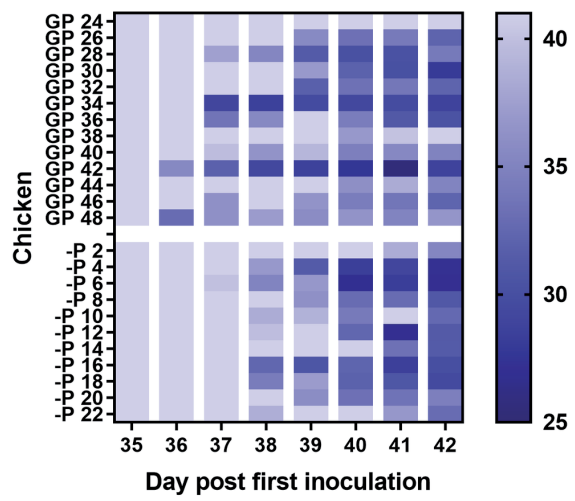

C Results C. psittaci PCR cloacal swabs

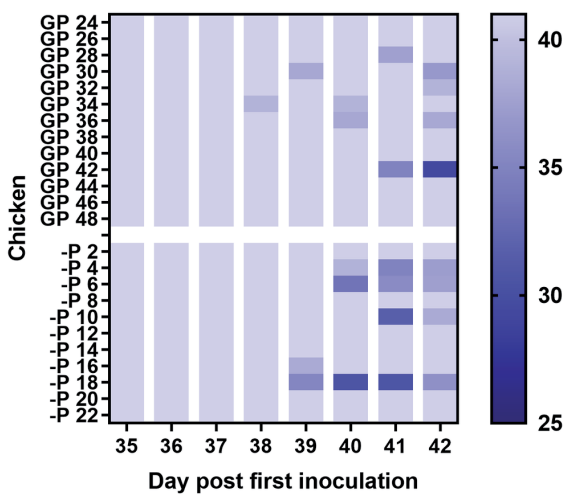

B Results C. psittaci PCR throat swabs



- Group -P

- Group GP

D Results C. psittaci PCR cloacal swabs

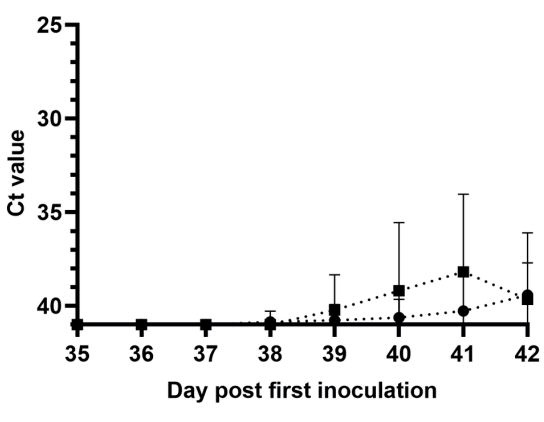

- Group -P $\quad$ - Group GP

Fig. 3. C. psittaci PCR results of group GP and -P

In A individual results of the C. psittaci PCR of throat swabs per timepoint are depicted in a heatmap. The darker the colour, the lower the Ct value as shown in the colour scale at the right side. Ct values $>40$ or no $P C R$ signal are shown as $C t 41$. B shows the mean Ct value of the throat swabs pet timepoint per group (of the C. psittaci PCR). The error bar indicates the SD. On the Y-axis the cycle treshold (Ct) value is depicted. The $Y$-axis has been rotated and $C t$ values $>40$ or no PCR signal are shown as $C t 41$. In $C$ individual results of the C. psittaci PCR of the cloacal swabs per timepoint are depicted in a heatmap. The darker the colour, the lower the Ct value as shown in the colour scale at the right side. Ct values $>40$ or no PCR signal are shown as Ct 41. D shows the mean Ct value of cloacal swabs in time per group (of the C. psittaci PCR). The error bar indicates the SD. On the $Y$-axis the cycle treshold $(C t)$ value is depicted. The $Y$-axis has been rotated and $C t$ values $>40$ or no $P C R$ signal are shown as $C t 41$.

In both group GP and -P, no clinical signs were observed during part 2 of the experiment based on the scoring card criteria. At necropsy, enlarged spleens were observed in 12/13 chickens of group GP and 10/11 of group -P. (Fig. 4B) Histological examination of the spleen showed a marked hyperplasia of both white and red pulp. (Fig.. 4C) The hyperplasia of the white pulp included both the peri-arteriolar lymphocyte sheath (PALS) as well as 
the peri-ellipsoid sheath (PELS). In group -P one chicken showed diffuse small white spots on the liver. Histological examination revealed a multifocal hepatitis with small foci of coagulation necrosis and influx of heterophils (Fig. S5). In another chicken the airsacs had a glazy appearance which was diagnosed by histopathology as an exsudative aerosacculitis. The presence of chlamydial antigen however could (not) be confirmed with immunohistochemistry in any of the tissues examined.

In group GP, C. psittaci was detected with PCR in 10/13 airsac samples (median Ct 36), 12/13 lung samples (median Ct 36.8), 12/13 liver samples (median Ct 33.7), 12/13 spleen samples (median Ct 30.1), 10/13 kidney samples (median Ct 36.7), 7/13 ileum samples (median Ct 38.9), 5/13 caecum samples (median Ct 41) and 6/13 samples of the caecal tonsil (median Ct 41). In group -P, C. psittaci was detected with PCR in 9/11 airsac samples (median Ct 34.4), 11/11 lung samples (median Ct 33.4), 11/11 liver samples (median Ct 32.3), 11/11 spleen samples (median Ct 29.4), 11/11 kidney samples (median Ct 35.9), 8/11 ileum samples (median Ct 36.4), 7/11 caecum samples (median Ct 37.6) and 8/11 samples of the caecal tonsil (median Ct 37.1). Fig. 4A shows the tissue dissemination patterns in group GP and -P overlapped. Overall there was a significant difference between the $\mathrm{Ct}$ values of the groups GP and $-P\left(X^{2}=5.83, p=0.016\right)$.

In group GP, one chicken remained C. psittaci PCR negative in throat swabs, cloacal swabs, and tissue samples during the entire experiment.
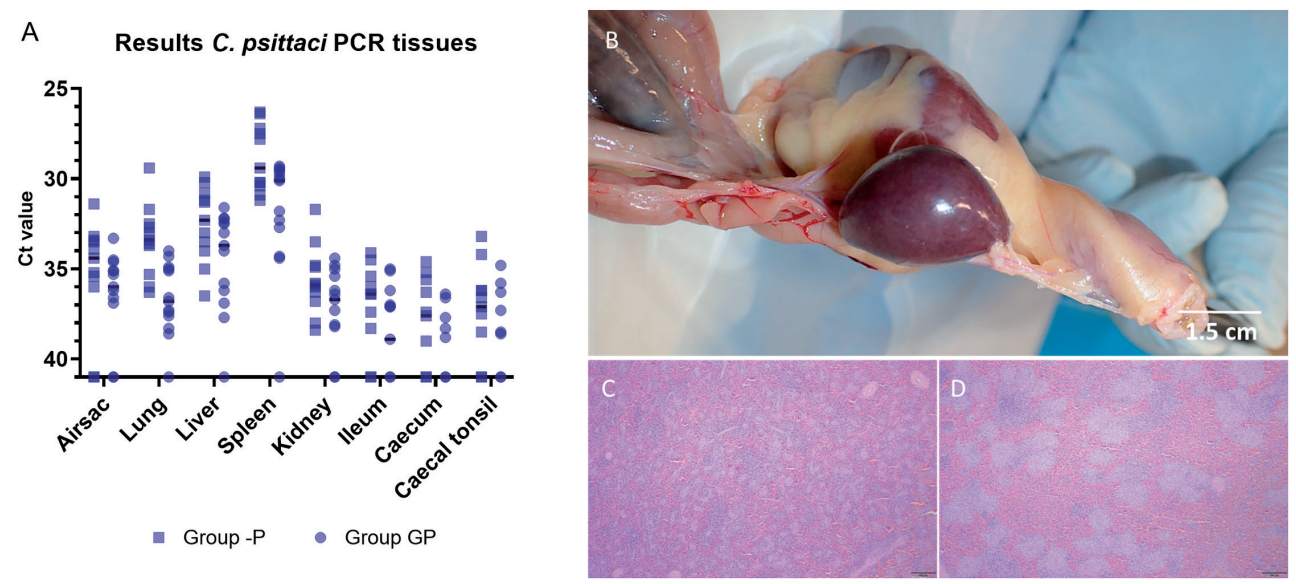

Fig. 4. Results of tissues in group GP and -P

A: the C. psittaci PCR results of tissue samples are shown. On the $Y$-axis the cycle treshold $(C t)$ value is depicted. The $Y$-axis has been rotated and $C t$ values $>40$ or no PCR signal are shown as $C t 41$. The bar indicates the median. B: macroscopic enlargement of the spleen in a C. psittaci infected animal. C and $D$ : histological examination of the spleen, same magnification. Notice the pronounced hyperplasia of the red and white pulp in the C. psittaci infected animal (D) compared to the animal infected with $C$. gallinacea (C). 
Secondary inoculations with C.gallinacea NL_F725 (group GG and -G)

Samples of the C.gallinacea NL_F725 infected groups (GG and -G) were only tested with the Chlamydiacea PCR, because no strain specific real-time PCR was available for $C$. gallinacea NL_F725 or NL_G47. In the group that was initially inoculated with C. gallinacea NL_G47 and subsequently inoculated with C. gallinacea NL_F725 (GG) significant reduced cloacal shedding was observed (Fig. $5 A$ and $B$ ) as compared to group $-G\left(x^{2}=35.6, p<0.001\right)$. In group GG PCR based cloacal shedding decreased in time, but in the control group $(-G)$ shedding increased (Fig. 5B). At the end of the experiment at day 42, 2/13 cloacal swabs tested positive $(\mathrm{Ct}<40)$ in group $\mathrm{GG}$, while all $(11 / 11)$ cloacal swabs in group $-\mathrm{G}$ tested positive (Fig. 5A).

At necropsy no pathological lesions were observed in group GG and - G. In group GG 5/13 chickens tested PCR positive in the ileum (median Ct 41), 10/13 in the caecum (median Ct 34.2) and 12/13 in the caecal tonsil (median Ct 29.9). In group -G, 11/11 chickens tested positive in the ileum, caecum and caecal tonsil (median $\mathrm{Ct} 25.2,23.7$ and 22.5 respectively) (Fig. 5C). In both group GG and -G, one chicken tested positive in the airsac (Ct 32.5 and 37 respectively). All other tissue samples tested PCR negative.

A

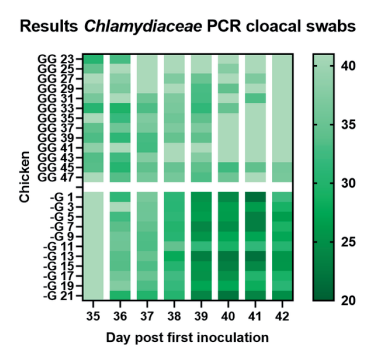

B

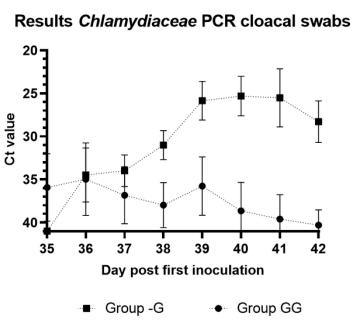

C Results Chlamydiaceae PCR tissues



Fig. 5. Chlamydiaceae PCR results of group GG and -G

In A individual results of the Chlamydiacea PCR of the cloacal swabs per timepoint are depicted in a heatmap. The darker the colour, the lower the $\mathrm{Ct}$ value as shown in the colour scale at the right side. $\mathrm{Ct}$ values $>40$ or no PCR signal are shown as Ct 41. B shows the mean Ct value of cloacal swabs in time per group (of the C. psittaci PCR). The error bar indicates the SD. On the Y-axis the cycle treshold $(C t)$ value is depicted. The $Y$-axis has been rotated and Ct values $>40$ or no PCR signal are shown as Ct 41. In $C$ the Chlamydiacea $P C R$ results of tissue samples are shown. The $Y$-axis has been rotated and $C t$ values $>40$ or no $P C R$ signal are shown as $C t 41$. The bar indicates the median.

\subsubsection{Differences between C. psittaci and C. gallinacea inoculation}

In the C. gallinacea inoculated control group (-G) and the C. psittaci inoculated control group (-P) the shedding and tissue dissemination pattern was different. In group - $G$ cloacal shedding was higher than in group -P and the start of the excretion was different: the $C$. 
gallinacea inoculated group started shedding on day 1 post inoculation (day 36), while the $C$. psittaci inoculated group started shedding only on day 4 after inoculation (day 39) (Fig. 6A). In group -P, shedding mainly occurred in throat swabs. In group -G throat swabs were not collected. In tissues Chlamydia was mainly detected in the ileum, caecum and caecal tonsil in all chickens of the -G group. In the -P group Chlamydia could be detected in all tissues, but the lowest $\mathrm{Ct}$ values were detected in the spleen and the highest in the gut in contrast to the results of the $-\mathrm{G}$ group (Fig. 6B).

A Shedding results Chlamydiaceae PCR



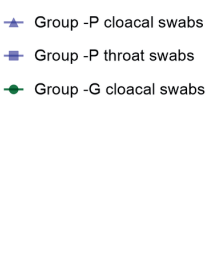

B

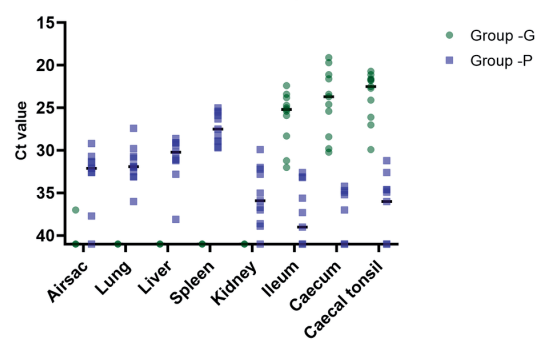

Fig. 6. Chlamydiaceae $P C R$ results of group - $G$ and $-P$

A shows the mean Ct value of swabs in time per group of the Chlamydiaceae PCR. The error bar indicates the SD. On the Y-axis the cycle treshold $(C t)$ value is depicted. The $Y$-axis has been rotated and $C t$ values $>$ 40 or no $P C R$ signal are shown as Ct 41. In B the Chlamydiaceae PCR results of tissue samples are shown of group - $G$ and group -P. The $Y$-axis has been rotated and $C t$ values $>40$ or no $P C R$ signal are shown as Ct 41. The bar indicates the median.

Summarising, chickens were succesfully infected with C. gallinacea NL_G47 in part 1 of the experiment while the controls remained Chlamydia negative. In part 2 no difference in C. psittaci shedding was observed between the group that was initially inoculated with C. gallinacea NL_G47 and subsequently infected with C. psittaci (GP) as compared to the control group that was subsequently inoculated with $C$. psittaci (-P). In the group that was initially inoculated with C. gallinacea NL_G47 and subsequently inoculated with $C$. gallinacea NL_F725 (GG) significant reduced cloacal shedding was observed as compared to the control group (-G). Furthermore, C. psittaci was mainly excreted via the throat and detected in systemic organs such as the spleen, while C. gallinacea was mainly detected in the gut. In none of the four groups clinical signs were observed based on the scoring card criteria. 


\section{Discussion}

In 2018 a high prevalence of C. gallinacea was detected on Dutch layer farms, but C. psittaci was absent in contrast to earlier studies in surrounding countries $(6,9,11)$. We hypothesized that the absence of $C$. psittaci could possibly be explained by cross protection between C. gallinacea and C. psittaci. This idea was also driven by the fact that cross reactive antibodies between chlamydial species are known to occur, because of the close structural similarity among some of the major surface antigens (12). To investigate whether an infection with C. gallinacea could protect against an infection with C. psittaci, chickens were first inoculated with C. gallinacea NL_G47 and subsequently inoculated with either C. gallinacea NL_F725 or with C. psittaci NL_Borg. The inoculations did not result in a difference in shedding or tissue dissemination of $C$. psittaci between the group that did not receive a first inoculation and the group that did receive a first inoculation with C. gallinacea, so cross protection was not observed.

We did observe a difference in tissue dissemination and shedding pattern between the groups that were inoculated with C. gallinacea and C. psittaci. In both groups the inoculation route was slightly different: the $C$. gallinacea groups were inoculated orally which resulted in an infection of the gut, while the $C$. psittaci groups received an oronasal inoculation that caused a more systemic infection (i.a. of the spleen). In C. gallinacea, the oral route is the main route of transmission for $C$. gallinacea and transmission via the respiratory route could not be proven (17). Considering C. psittaci, infections via the respiratory route are more efficient than infections via the oral route (23). The different porte d'entree and subsequent localization of the infection is probably caused by a difference in tissue tropism of C. gallinacea and C. psittaci.

A difference in tissue tropism between C. gallinacea and C. psittaci could also partially explain why cross protection was not observed. The successful clearance of a Chlamydia infection probably depends on both a local and systemic, cell-mediated and humoral response with neutralizing antibodies that act either by inhibiting binding to epithelial cells or activation of complement, leading to lysis of the Chlamydia membrane $(24,25)$. In our study, a rise in anti-Chlamydia antibodies was measured after the first inoculation with C. gallinacea, but, after the second inoculation, C. psittaci could be detected in organs such as the spleen and liver, suggesting circulating neutralizing antibodies against C. psittaci were not elicited or could not prevent infection. In a C. trachomatis vaccine study, neutralizing antibodies against the variable domain 4 (VD4) of MOMP were very important in preventing infection in a mouse model, but this effect was also specific (24). Small differences in the amino acid sequence of the epitope could already prevent neutralization $(26,27)$. Additional in-vitro studies are therefore needed to investigate 
if C. gallinacea infection elicits neutralizing antibodies and if these antibodies have a neutralizing effect on C. psittaci.

If neutralizing antibodies are not elicited or do no neutralize C. psittaci, (partial) cross protection against $C$. psittaci would depend on local immune responses. However, a local response might not be effective because of the observed difference in tissue tropism between C. gallinacea and C. psittaci. In the group that received a first inoculation with C. gallinacea NL_G47 and a subsequent inoculation with C. gallinacea NL_F725 cross protection was observed. This could support the possible role of the local immune response, although neutralizing antibodies could have an effect as well. This would require further investigation as already concluded.

In addition, the difference in shedding pattern between C. gallinacea and C. psittaci could cause differences in transmission, which might alternatively explain why $C$. gallinacea was highly prevalent and C. psittaci was not detected in the prevalence study (6). At first, the degree of shedding of C.gallinacea appeared to be higher which will facilitate transmission and, second, the main route of transmission is different. In chickens, the respiratory route is likely to be more important for C. psittaci based on previous studies that compared inoculation routes (23), and the higher degree of shedding via the throat, although this might depend on the stage of infection. Pharyngeal shedding is mainly higher during the early part infection until day eight (28); our experiment ended at seven days post inoculation with C. psittaci. To further understand how these differences in infection dynamics could affect prevalence, comparative transmission studies with $C$. gallinacea and C. psittaci in chickens would be of added value. These studies should also take into account sampling strategy regarding the differences in shedding pattern.

Before the second inoculation with C. psittaci or C. gallinacea NL_F725, 10/13 chickens where still shedding Chlamydia in both groups. It was expected shedding would decrease after transport to a clean environment, because it was thought part of the shedding might be explained by passive transfer of $C$. gallinacea (DNA) from the environment. However, this effect was not observed and the transport of the chickens as a stress factor might have had an enhancing effect on cloacal shedding as known for C. psittaci (29).

The remaining presence of NL_G47 at the start of the second part of the experiment could have underestimated the effect on shedding in the GG group, because NL_G47 and NL_ F725 could not be differentiated with the Chlamydiaceae PCR. On the other hand, it could also have caused a type of competitive exclusion in which the local presence of NL_G47 prevented NL_F725 to enter gut epithelial cells (30). This kind of effect seems unlikely, because it has not been described before in Chlamydia and might have been observed in both the GG and GP group. However, cloacal shedding and colonization of the gut in $C$. 
psittaci infection (group -P) was in general much lower than in C. gallinacea infection (-G) possibly due to a difference in tissue tropism as discussed above.

In conclusion, a prior $C$. gallinacea infection, does partially protect against a new $C$. gallinacea infection based on the PCR based results of cloacal shedding. However, a prior infection with $C$. gallinacea is not protective against a subsequent infection with C. psittaci based on shedding and tissue dissemination. The absence of $C$. psittaci in an earlier prevalence study (6) can therefore not be explained by such cross protection. The question remains how often C. psittaci is introduced in chickens flocks, how well infections can be transmitted and whether infections might go unnoticed as no clinical signs were observed during our experiment. This would require future comparative transmission studies.

\section{Funding}

This work was supported by the Dutch Ministry of Agriculture, Nature and the Environment (grant WOT-01-002-005.02, WOT-01-01-002-005.13 and KB-21-006-022).

\section{Acknowledgements}

The authors acknowledge the animal care takers from WBVR for their assistance during the animal experiments, Sebastiaan van der Broek, Irene Oud and Marianne Vahl from WBVR for their technical assistance in the analyses of the samples. The authors would also like to thank Fimme Jan van der Wal and Ruth Bossers for the development of the ELISA, and Monique Bakker for her help with the design of Fig. 1. 


\section{Supporting information}

\section{Supporting file 1. Scoring card clinical signs}

\begin{tabular}{|c|c|c|c|}
\hline & No signs (0) & Mild (1) & Severe (2) \\
\hline Mental state & $\begin{array}{l}\text { Active, makes noise, } \\
\text { responds to environment } \\
\text { or handling }\end{array}$ & $\begin{array}{l}\text { Less active, bulging } \\
\text { (with feathers upright), } \\
\text { but responding to } \\
\text { environment }\end{array}$ & $\begin{array}{l}\text { No response to } \\
\text { environment, lying, } \\
\text { retreating, hardly to no } \\
\text { response to handling, } \\
\text { stopped eating and } \\
\text { drinking }\end{array}$ \\
\hline Head & $\begin{array}{l}\text { No discharge from nose } \\
\text { or eye, no red eyes }\end{array}$ & $\begin{array}{c}\text { Watery to mucous } \\
\text { discharge from eye and/ } \\
\text { or nose (tear stripe), red } \\
\text { eyes }\end{array}$ & $\begin{array}{c}\text { Severe mucous or } \\
\text { bloody discharge and/or } \\
\text { dense red, swollen eyes }\end{array}$ \\
\hline Upper airways & $\begin{array}{l}\text { No sneezing or shaking } \\
\text { with the head }\end{array}$ & Occasionally sneezing & $\begin{array}{l}\text { Frequent sneezing and } \\
\text { shaking of the head }\end{array}$ \\
\hline Lower airways & $\begin{array}{l}\text { No increased respiration } \\
\text { frequency or symptoms } \\
\text { of shortness of breath }\end{array}$ & $\begin{array}{l}\text { Slightly increased } \\
\text { respiration frequency } \\
\text { and / or noises such as } \\
\text { gargling and grating }\end{array}$ & $\begin{array}{c}\text { Clearly increased } \\
\text { breathing frequency, } \\
\text { open mouth, stretched } \\
\text { neck, symptoms of } \\
\text { shortness of breath, } \\
\text { noises such as gargling } \\
\text { and rattling }\end{array}$ \\
\hline Gait and balance & $\begin{array}{c}\text { Normal gait, no } \\
\text { uncoordinated } \\
\text { movements or tremors }\end{array}$ & $\begin{array}{c}\text { Difficulty with } \\
\text { coordination when } \\
\text { standing up, can } \\
\text { walk but seems to } \\
\text { have more difficulty } \\
\text { with coordination of } \\
\text { movements } \\
\end{array}$ & $\begin{array}{c}\text { Disturbed balance, } \\
\text { difficulty walking or } \\
\text { paralysis, twisted neck, } \\
\text { walking in circles, severe } \\
\text { muscle tremors }\end{array}$ \\
\hline Feces & $\begin{array}{c}\text { Normal chicken feces, no } \\
\text { abnormal consistency } \\
\text { or color }\end{array}$ & $\begin{array}{l}\text { Feces with abnormal color } \\
\text { (green to yellow) and/or } \\
\text { consistency (wetter) }\end{array}$ & $\begin{array}{l}\text { Feces with abnormal color, } \\
\text { consistency and quantity, } \\
\text { presence of blood }\end{array}$ \\
\hline
\end{tabular}




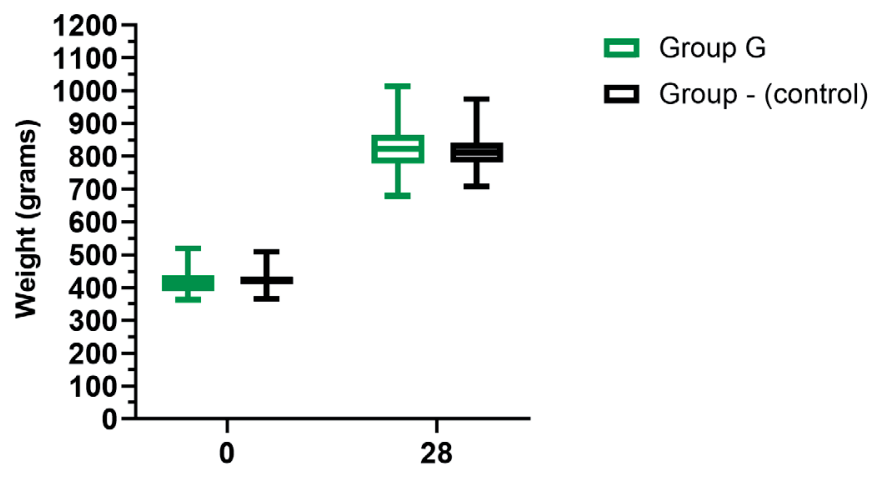

Day post first inoculation

\section{Supporting file 2. Differences in weight in group $\mathbf{G}$ and - (control)}

Differences in weight (grams) in group $G$ and group - (control) at the start of part 1 of the experiment and after 28 days is shown in a boxplot. The whiskers plot down to the smallest value and up to the largest and the box extends from the $25^{\text {th }}$ to $75^{\text {th }}$ percentile.

A

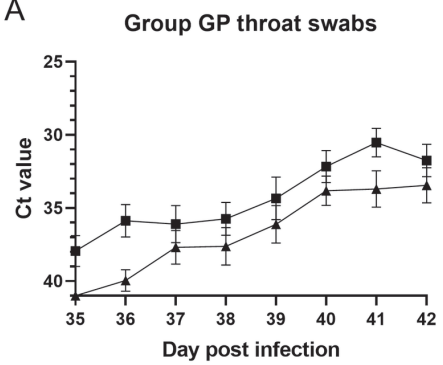

C

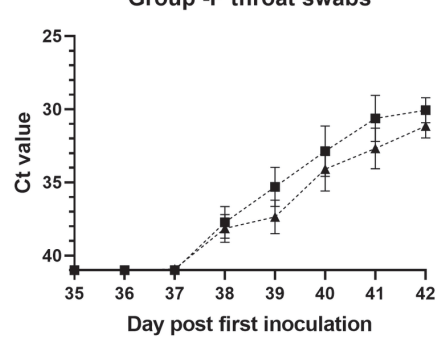

B

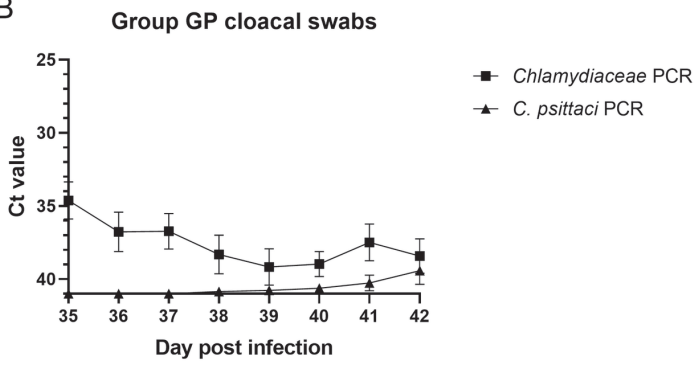

D

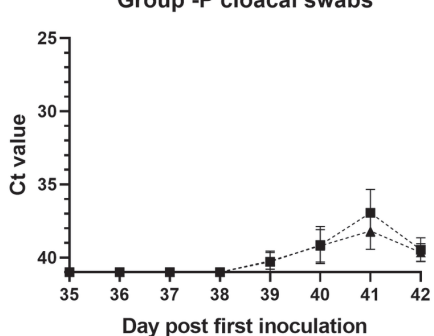

- Chlamydiaceae PCR

- C. psittaci PCR

\section{Supporting file 3. Differences between the Chlamdyiaceae PCR and C. psittaci PCR}

$A$ and $B$ show the mean Ct value of throat and cloacal swabs in time of group GP in the Chlamydiaceae $P C R$ and C. psittaci PCR. C and D show the mean Ct value of throat and cloacal swabs in time of group - $P$ in the Chlamydiaceae PCR and C. psittaci PCR. The error bar indicates the SEM in all figures. On the Y-axis the cycle treshold $(\mathrm{Ct})$ value is depicted. The $Y$-axis has been rotated and $\mathrm{Ct}$ values $>40$ or no PCR signal are shown as $\mathrm{Ct} 41$. 


\section{References}

1. Zareba-Marchewka K, Szymanska-Czerwinska M, Niemczuk K. Chlamydiae - What's New? J Vet Res. 2020;64(4):461-7.

2. Kaleta EF, Taday EM. Avian host range of Chlamydophila spp. based on isolation, antigen detection and serology. Avian pathology : journal of the WVPA. 2003;32(5):435-61.

3. Harkinezhad T, Geens T, Vanrompay D. Chlamydophila psittaci infections in birds: a review with emphasis on zoonotic consequences. Veterinary microbiology. 2009;135(1-2):68-77.

4. Sachse K, Laroucau K, Riege K, Wehner S, Dilcher M, Creasy HH, et al. Evidence for the existence of two new members of the family Chlamydiaceae and proposal of Chlamydia avium sp. nov. and Chlamydia gallinacea sp. nov. Systematic and applied microbiology. 2014;37(2):79-88.

5. Laroucau K, Vorimore F, Aaziz R, Berndt A, Schubert E, Sachse K. Isolation of a new chlamydial agent from infected domestic poultry coincided with cases of atypical pneumonia among slaughterhouse workers in France. Infection, genetics and evolution : journal of molecular epidemiology and evolutionary genetics in infectious diseases. 2009;9(6):1240-7.

6. Heijne M, van der Goot JA, Fijten H, van der Giessen JW, Kuijt E, Maassen CBM, et al. A cross sectional study on Dutch layer farms to investigate the prevalence and potential risk factors for different Chlamydia species. PloS one. 2018;13(1):e0190774.

7. Hulin V, Oger S, Vorimore F, Aaziz R, de Barbeyrac B, Berruchon J, et al. Host preference and zoonotic potential of Chlamydia psittaci and C. gallinacea in poultry. Pathogens and disease. 2015;73(1):1-11.

8. Guo W, Li J, Kaltenboeck B, Gong J, Fan W, Wang C. Chlamydia gallinacea, not C. psittaci, is the endemic chlamydial species in chicken (Gallus gallus). Scientific reports. 2016;6:19638.

9. Lagae S, Kalmar I, Laroucau K, Vorimore F, Vanrompay D. Emerging Chlamydia psittaci infections in chickens and examination of transmission to humans. Journal of medical microbiology. 2014;63(Pt 3):399-407.

10. Dickx V, Geens T, Deschuyffeleer T, Tyberghien L, Harkinezhad T, Beeckman DS, et al. Chlamydophila psittaci zoonotic risk assessment in a chicken and turkey slaughterhouse. Journal of clinical microbiology. 2010;48(9):3244-50.

11. Yin L, Kalmar ID, Lagae $S$, Vandendriessche $S$, Vanderhaeghen W, Butaye $P$, et al. Emerging Chlamydia psittaci infections in the chicken industry and pathology of Chlamydia psittaci genotype $B$ and $D$ strains in specific pathogen free chickens. Veterinary microbiology. 2013;162(2-4):740-9.

12. Sachse K, Rahman KS, Schnee C, Muller E, Peisker M, Schumacher T, et al. A novel synthetic peptide microarray assay detects Chlamydia species-specific antibodies in animal and human sera. Scientific reports. 2018;8(1):4701.

13. Heijne $M$, Jelocnik $M$, Umanets A, Brouwer MSM, Dinkla A, Harders F, et al. Genetic and phenotypic analysis of the pathogenic potential of two novel Chlamydia gallinacea strains compared to Chlamydia psittaci. Scientific reports. 2021;11(1):16516. 
14. Heijne M, van der Goot J, Buys $H$, Dinkla A, Roest HJ, van Keulen L, et al. Pathogenicity of Chlamydia gallinacea in chickens after oral inoculation. Veterinary microbiology. 2021:109166.

15. SPEARMAN C. THE METHOD OF 'RIGHT AND WRONG CASES' ('CONSTANT STIMULI') WITHOUT GAUSS'S FORMULAE. British Journal of Psychology, 1904-1920. 1908;2(3):227-42.

16. Kärber G. Beitrag zur kollektiven Behandlung pharmakologischer Reihenversuche. NaunynSchmiedebergs Archiv für experimentelle Pathologie und Pharmakologie. 1931;162(4):480-3.

17. You J, Wu Y, Zhang X, Wang X, Gong J, Zhao Z, et al. Efficient fecal-oral and possible vertical, but not respiratory, transmission of emerging Chlamydia gallinacea in broilers. Veterinary microbiology. 2019;230:90-4.

18. Takahashi T, Takashima I, Hashimoto N. Shedding and transmission of Chlamydia psittaci in experimentally infected chickens. Avian diseases. 1988;32(4):650-8.

19. Andersen AA. Comparison of pharyngeal, fecal, and cloacal samples for the isolation of Chlamydia psittaci from experimentally infected cockatiels and turkeys. Journal of veterinary diagnostic investigation : official publication of the American Association of Veterinary Laboratory Diagnosticians, Inc. 1996;8(4):448-50.

20. Ehricht R, Slickers P, Goellner S, Hotzel H, Sachse K. Optimized DNA microarray assay allows detection and genotyping of single PCR-amplifiable target copies. Molecular and cellular probes. 2006;20(1):60-3.

21. Pantchev A, Sting R, Bauerfeind R, Tyczka J, Sachse K. New real-time PCR tests for speciesspecific detection of Chlamydophila psittaci and Chlamydophila abortus from tissue samples. Veterinary journal. 2009;181(2):145-50.

22. Team RC. A language and environment for statistical computing. Vienna, Austria: R Foundation for Statistical Computing; 2019.

23. Takahashi T, Takashima I, Hashimoto N. A chicken model of systemic infection with Chlamydia psittaci: comparison of the virulence among avian and mammalian strains. Nihon juigaku zasshi The Japanese journal of veterinary science. 1988;50(3):622-31.

24. Olsen AW, Lorenzen EK, Rosenkrands I, Follmann F, Andersen P. Protective Effect of Vaccine Promoted Neutralizing Antibodies against the Intracellular Pathogen Chlamydia trachomatis. Frontiers in Immunology. 2017;8(1652).

25. Phillips S, Quigley BL, Timms P. Seventy Years of Chlamydia Vaccine Research - Limitations of the Past and Directions for the Future. Frontiers in microbiology. 2019;10:70.

26. Kari L, Whitmire WM, Carlson JH, Crane DD, Reveneau N, Nelson DE, et al. Pathogenic Diversity among Chlamydia trachomatis Ocular Strains in Nonhuman Primates Is Affected by Subtle Genomic Variations. The Journal of infectious diseases. 2008;197(3):449-56.

27. Kari L, Whitmire WM, Crane DD, Reveneau N, Carlson JH, Goheen MM, et al. Chlamydia trachomatis native major outer membrane protein induces partial protection in nonhuman primates: implication for a trachoma transmission-blocking vaccine. The Journal of immunology. 2009;182(12):8063-70. 
28. Yin L, Lagae S, Kalmar I, Borel N, Pospischil A, Vanrompay D. Pathogenicity of low and highly virulent Chlamydia psittaci isolates for specific-pathogen-free chickens. Avian diseases. 2013;57(2):242-7.

29. Balsamo G, Maxted AM, Midla JW, Murphy JM, Wohrle R, Edling TM, et al. Compendium of Measures to Control Chlamydia psittaci Infection Among Humans (Psittacosis) and Pet Birds (Avian Chlamydiosis), 2017. J Avian Med Surg. 2017;31(3):262-82.

30. Schneitz C. Competitive exclusion in poultry--30 years of research. Food Control. 2005;16(8):657-67. 


\section{CHAPTER 6}




\section{General discussion}


Chlamydia gallinacea and Chlamydia psittaci are both obligate intracellular bacteria that can infect poultry. In poultry, C. psittaci was considered the main chlamydial species until the discovery of C. gallinacea in 2009 (1). C. gallinacea is widespread in chickens, but research into its pathogenic potential is limited thus far (2). Furthermore, the prevalence of C. psittaci in chickens might be higher than previously thought (3). Therefore, the major aims of this thesis focussed on the following research questions:

1. What is the prevalence of Chlamydia in Dutch poultry?

2. What is the primary pathogenicity of $C$. gallinacea?

3. Could a previous infection with C. gallinacea protect against an infection with C. psittaci?

The prevalence of Chlamydia in Dutch layers was investigated in Chapter 2. C. gallinacea DNA was detected in pooled faecal samples on 71 of the 151 layer farms, but C. psittaci DNA was not detected. No association between clinical signs (i.e. respiratory symptoms, nasal and ocular discharge, mortality) and the presence of C. gallinacea was found.

In Chapter 3, two novel C. gallinacea strains (NL_G47 and NL_F725) were isolated from the caeca of apparently healthy chickens. Genomic analysis showed both strains were unique and possessed the hallmark genes coding for known and potential virulence factors as found in C. psittaci, albeit to a reduced number of orthologs or alleles. Phenotypic analysis in embryonated specific pathogen free (SPF) eggs revealed C. gallinacea induced mortality, but to a lower extent than C. psittaci.

Subsequent experiments with C. gallinacea strain NL_G47 in six-week old SPF layers in Chapter 4, confirmed observations from field studies that $C$. gallinacea infection does not result in acute clinical disease. In this study layers were orally inoculated which resulted in throat- and cloacal shedding and infection of epithelial cells of the jejunum, ileum and caecum without signs of clinical disease, nor in macroscopic or histologic signs of inflammation. At day 11 post inoculation, chlamydial antigen was co-localized within macrophages in the lamina propria and follicular dendritic cells in the caecal tonsil and, from day 7 onwards, a rise in antibody titre was shown.

After the finding in Chapter $\mathbf{2}$ that C. gallinacea was highly prevalent in Dutch layer farms, we hypothesized in Chapter $\mathbf{5}$ that the absence of $C$. psittaci could be explained by cross protection between C. gallinacea and C. psittaci. Chickens were therefore first inoculated with C. gallinacea NL_G47 and subsequently inoculated with either C. gallinacea NL_F725 or with C. psittaci NL_Borg. The inoculations did not result in a difference in shedding or tissue dissemination of $C$. psittaci between the group that did not receive a first inoculation and the group that did receive a first inoculation with $C$. gallinacea. Thus, the absence of C. psittaci in the prevalence study could not be explained by cross protection. However, 
a prior C. gallinacea infection did partially protect against a new C. gallinacea infection based on the PCR results of cloacal shedding.

\section{Chlamydia infections in poultry other than layers}

In this thesis, data were mostly collected in layers, but what do these data tell about broilers or other poultry species? Poultry is an umbrella term for domesticated bird species, such as chickens, ducks, geese and turkeys, and usually further subdivided into layers and broilers based on the production goal (eggs or meat). In the Netherlands, about $90 \%$ of poultry is kept in specialized poultry farms with a total number of about 100 million animal places in 2020 (CBS Statline). The majority of these animals are chickens with approximately 44 million layers, housed at 856 farms and 49 million broilers, housed at 637 farms (Table 1). Chickens are therefore the most important part of poultry industry in the Netherlands, but can data from layers be extrapolated to broilers?

Table 1. Poultry farms and number of animal places in the Netherlands in 2020 (CBS Statline)

\begin{tabular}{lcc}
\hline Poultry and farm type & Number of farms (n) & Number of animals (n) \\
\hline Chickens; layers & 856 & $43,165,986$ \\
\hline Chickens; layers; parent flocks & 48 & $1,674,306$ \\
\hline Chickens; broilers & 637 & $49,228,507$ \\
\hline Chickens; broilers; parent flocks & 248 & $7,794,318$ \\
\hline Turkeys & 31 & 585,134 \\
\hline Ducks & 50 & 819,191 \\
\hline Other poultry species & 12 & 33,285 \\
\hline
\end{tabular}

In chickens, the selection for two different production goals (meat versus eggs) has resulted in two different farming systems with two different animal types. Broilers are fast growing animals that are slaughtered at six weeks of age, while layers grow more slowly and are slaughtered at about 80 weeks of age. Therefore, prevalence data cannot readily be extrapolated, although a lower prevalence would be expected in broilers due to their shorter lifespan. This was already observed in a Mexican cross-sectional study, where layer flocks indeed had a higher risk of being Chlamydia positive than broiler flocks, although farming systems might differ between the Netherlands and Mexico (4). In a more recent Dutch surveillance study in broilers, Chlamydiaceae DNA could not be detected in pooled faecal samples at 90 investigated farms (unpublished results). Although exact prevalence data cannot be extrapolated, layers can be considered an indicator for the presence of Chlamydia in chickens in general. 
Differences between broilers and layer systems might also result in differences in disease susceptibility and disease outcome in Chlamydia infection. Studies investigating these differences have not been performed, but both C. gallinacea and C. psittaci have been detected in broilers and layers $(3,4)$. Furthermore, in an experimental study with $C$. gallinacea in broilers, it was observed that infection might result in reduced weight gain (5). This effect was not observed in SPF layers between six to ten weeks of age (Chapter 5) and is most likely due to the difference in growth between broilers and layers. In layers, it would be more logical to investigate other production parameters such as egg production at a later stage.

Extrapolation of data to other poultry species is even more difficult. A French study investigating the prevalence of Chlamydia in different poultry species has shown $C$. gallinacea was mostly detected in chickens and guinea fowl, while C. psittaci was more prevalent in ducks (6). From C. psittaci infections in different bird species, it is known that susceptibility and disease outcome depends on both the host, C. psittaci strain and environmental factors (7). For example, in turkeys, severe systemic infections including mortality have been described $(8,9)$, while ducks seem to be less susceptible to $C$. psittaci infection and are mainly subclinically infected $(10,11)$. Hence, separate prevalence studies would be required to estimate the prevalence of Chlamydia in turkeys and ducks in the Netherlands. Moreover, based on the data about $C$. psittaci, results about the pathogenesis of $C$. gallinacea in chickens cannot be translated to other poultry species. Nonetheless, current prevalence data suggest that other poultry species are less susceptible to $C$. gallinacea infection $(5,6,12)$.

\section{C. gallinacea, a pathogen at all?}

Although potential virulence factors are present in the C. gallinacea genome and infection of embryonated eggs can result in mortality, acute clinical disease was not observed in layers in the prevalence study nor in the oral infection experiments. From that perspective C. gallinacea does not behave like a pathogen. However, chlamydial infections are intracellular by nature and notorious for causing long term or chronic health effects due to persistent infections (13-15). Hence, is there evidence that C. gallinacea might cause persistent infections?

Persistence refers to infections that cannot be cleared either at cell, organ, organism or population level. In Chlamydia research the different levels of persistence are often confounded with each other (16). Furthermore, persistence can coincide at different levels. Chlamydia persistence at cell level means the replication cycle is interrupted and the bacteria turn into a viable but non-cultivable state (aberrant bodies) when facing 
a stress stimulus such as non-bactericidal antibiotic treatment, cytokine exposure or nutrient deprivation (15). This phenomenon can be considered a kind of dormancy and is mainly observed in vitro, but there is some evidence it might occur in vivo as well (15). Persistency at cell level has not been investigated for C. gallinacea, but is likely to occur in vitro in line with findings for other Chlamydia. It will be much more challenging to detect this type of persistence in vivo as current methodology might not be sensitive enough.

Persistence at organ, organism or population level usually refers to an infection that cannot be cleared, because of the continuing presence of susceptible cells or animals that can be infected. In Chlamydia, this type of persistence is seen in chronic gastrointestinal infections in which it is thought that local downregulation of the immune system prevents clearance of the infection $(14,17)$. C. gallinacea also resides in the gastrointestinal tract. In chapter 5, C. gallinacea could be detected in 20/26 cloacal swabs for at least 35 days after inoculation. In other studies, C. gallinacea was detected in the rectum at day 26 post infection (18), or for at least three months in cloacal swabs (5). In a recent transmission experiment (unpublished results) C. gallinacea DNA could be detected until four to five months after first detection in cloacal swabs of chickens in the exposed flock (Fig. 1, unpublished data). In this experiment two groups of 25 Chlamydia PCR negative layers were exposed to three C. gallinacea positive layers. Within two weeks after the introduction of the positive layers all cloacal swabs of the contact animals tested PCR positive. During the experiment shedding appeared to be intermittent in individual animals. After five months all cloacal swabs tested PCR negative at three consecutive time points with two week intervals. These results indicate C. gallinacea infections might persist for several months, but are cleared based on cloacal shedding. It was not investigated whether Chlamydia could still be present in the gut and subsequent cloacal shedding could be below the detection level. Furthermore, no apparent health effects were observed, although more subtle signs would have remained unnoticed as a C. gallinacea negative control group was not included. 


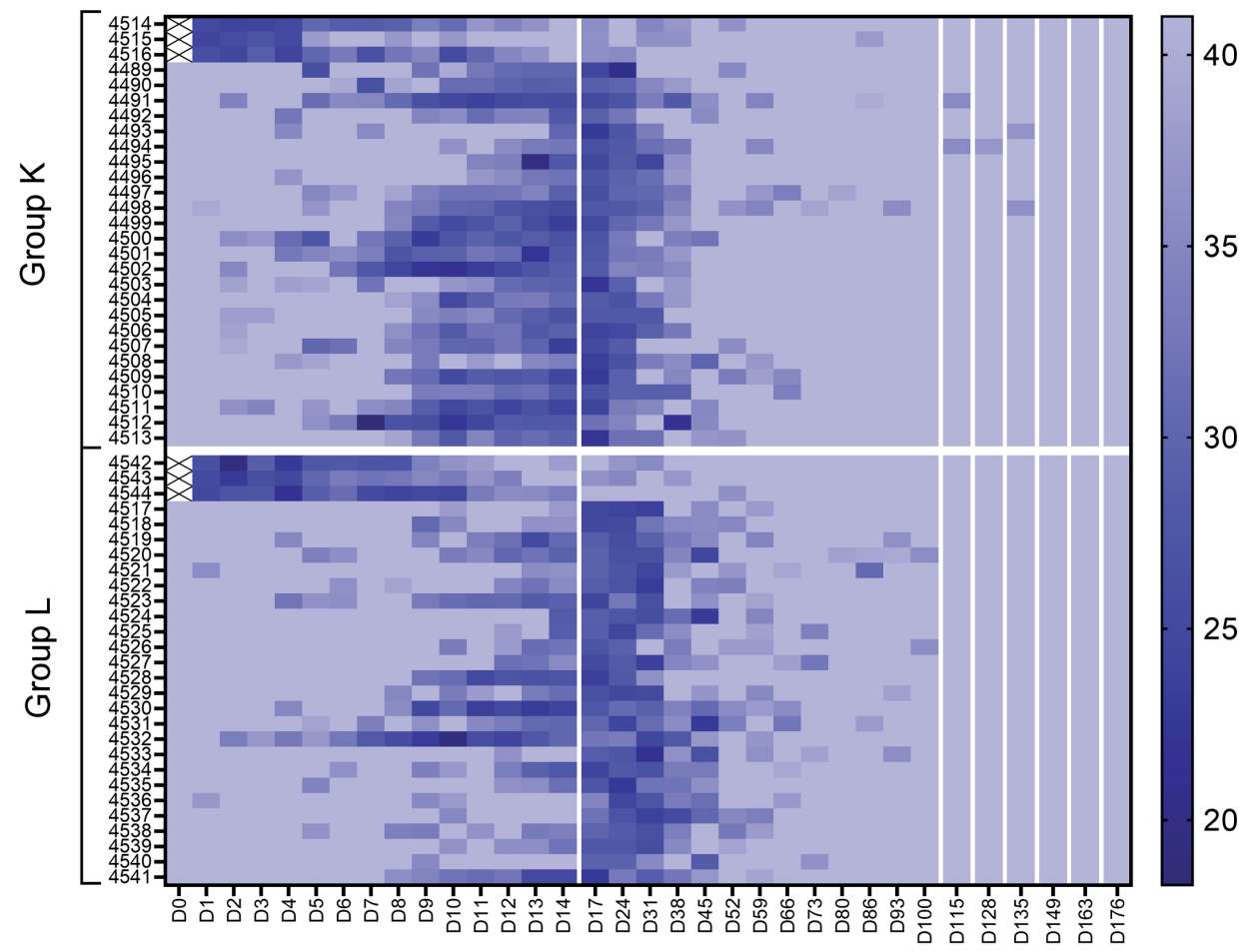

Fig. 1. PCR results of cloacal swabs in field transmission experiment

Chlamydiaceae PCR results of individual cloacal swabs per timepoint depicted in a heatmap. The darker the colour, the lower the Ct value as shown in the colour scale at the right side. Ct values $>40$ or no PCR signal are shown as $C t 41$. The chickens were housed in two separate pens (group $K$ and $L$ ) in one room. The upper three chickens in group $K$ and $L$ are the $C$. gallinacea positive chickens that were added after the sampling at day 0 . Chickens were sampled daily until day 14 , once a week until day 100 and at more irregular time intervals of one to two weeks from day 100 onwards.

Chronic health effects or adverse effects on production parameters caused by persistent infections, might be the result of (metabolic) costs due to a possible higher turnover of gut epithelial cells or an increase in adaptive immune response (19). Such an effect has been observed in broilers with reduced weight gain, although this cannot be considered long term considering the slaughter age of broilers (5). It would be more relevant to investigate an effect on egg production, also because $C$. gallinacea spreads rapidly and is highly prevalent in Dutch layers. However, this will be hard to achieve under field conditions as it might be very difficult (or impossible) to include flocks that remain C. gallinacea negative throughout their production cycle.

Moreover, even if $C$. gallinacea does negatively affect production in layers, it could be questioned whether the costs of a control strategy would outweigh the possible benefit as long as there is also no clear evidence of a zoonotic potential. A C. gallinacea vaccine is 
currently not available and might even have more negative side effects than an infection itself. Flock treatments with antibiotics are not easy to perform and very undesirable in view of the risks of development and spread of antibiotic resistance. In conclusion, it cannot be excluded that infections potentially have negative long term effects and C. gallinacea might still be considered a pathogen. However, based on current data in chickens it is not a pathogen of concern for which control or eradication seems indicated.

\section{A one health perspective on C. psittaci infections in chickens}

In our prevalence study, C. psittaci could not be detected in layers in the Netherlands (Chapter 2). In a more recent study (unpublished results), C. psittaci could also not be detected in 90 broiler flocks. Therefore, there is currently no evidence that the earlier reported increased risk of human pneumonia around poultry farms is associated with $C$. psittaci infections in chickens (20). Other studies investigating the association between poultry farms and human pneumonia could either not confirm the higher incidence, or, associated the higher incidence with more indirect causes such as exposure to air pollutants and endotoxins $(21,22)$.

The absence of $C$. psittaci in the prevalence study does not exclude any future introduction. Our study (Chapter 5) showed chickens can experimentally be infected with C. psittaci in line with findings of other experimental studies (23-27). Furthermore, we did not find an indication that chickens are protected against a $C$. psittaci infection due to a prior infection with C. gallinacea. C. psittaci is endemic in many bird species (7, 28-30), so introduction of $C$. psittaci might be possible via contact with infected wild birds. Transmission from wild birds to poultry has been experimentally shown in the past, when wild birds were considered a possible source of outbreaks in turkeys in the United States $(31,32)$. It is also likely that the risk of introduction will be higher on farms with outdoor housing as already known for Avian Influenza where wild birds play an important role in the epidemiology $(33,34)$. After introduction, C. psittaci infections might remain unnoticed as clinical signs are not always observed as shown in Chapter 5, but the question is how well C. psittaci can be transmitted between chickens.

C. psittaci can be transmitted from inoculated chickens to uninfected cagemates in an experimental setting (25). In this study, the infection in cagemates was less severe than in the inoculated chickens, raising the question whether the cagemates would have been able to transmit C. psittaci to other uninfected chickens. Unfortunately, data from field studies about C. psittaci infection dynamics in chickens are scarce. Most studies in chickens focus on the risks of zoonotic transmission at slaughterhouse level. The lack of field data might implicate C. psittaci infections are not efficiently transmitted between 
chickens. Finally, it has always been assumed that chickens are less susceptible to infection than other bird species $(11,35)$. Nevertheless, it would be of added value to confirm this experimentally, also from a One Health perspective. If transmission of $C$. psittaci between chickens is limited, the risk that chickens will be a future reservoir for human C. psittaci infections is also very limited.

\section{Final thoughts and future research}

From a One Health perspective, Chlamydiaceae infections in Dutch chickens are currently not a problem, although some questions remain to be answered. Most important is to investigate how well C. psittaci can be transmitted between chickens, also because poultry husbandry might change to more outdoor systems in the future which can increase the risk of introduction of a C. psittaci infection. Transmission studies should focus on naturally infected animals and at least take into account $C$. psittaci genotype A, B and D. Genotype $A$ is probably the most common C. psittaci genotype worldwide and detected in different animal species, including chickens, although it is classically associated with psittacine birds $(36,37)$. Genotype B is mostly found in homing and feral pigeons and shown to cause disease in chickens under experimental conditions $(23,24)$. In the Netherlands genotype $A$ and $B$ are the most frequently detected $C$. psittaci genotypes in humans and pet birds $(38,39)$. Genotype D should be included as it was used in our study in Chapter 2 and 5, caused severe outbreaks in turkeys and was shown to cause disease in chickens in other experimental studies $(23,24)$. Transmission studies will provide further insight into the susceptibility of chickens to C. psittaci infection and subsequent infection dynamics, which will help to determine whether chickens could be a potential reservoir for $C$. psittaci infections.

Furthermore, the prevalence of C. psittaci in ducks and turkeys in the Netherlands is still unknown. In turkeys, C. psittaci infections are mostly associated with severe clinical signs and high mortality (35). During an outbreak of C. psittaci on a Dutch turkey farm in the nineties, mortality up to $65 \%$ was reported (9). It is very unlikely that this type of infections will be missed. Some studies also indicate infections might be milder and can remain undetected $(40,41)$. Furthermore, it is important to note that there are no turkey slaughterhouses in the Netherlands. Most cases of poultry-to-human transmission are associated with slaughterhouses $(11,36)$.

In ducks infections are considered to be mainly subclinical $(10,11)$, but more severe infections have been reported as well (35). Currently, there are no indications from routine pathology at the Animal Health Service (Royal GD) that Chlamydia infections play a role in ducks in the Netherlands, but infections could be missed as Chlamydia will not be 
detected with routine bacteriological culture. Furthermore, a weak spatial association has been found between duck farms and human psittacosis cases in the Netherlands (42). This association should be treated with caution as the same study also reported an association with chicken processing plants, which seems unlikely based on the results from the prevalence studies. These data show it would be worthwhile to investigate the prevalence of C. psittaci in turkeys and ducks, with ducks being the most relevant species. It would be most convenient to use surveillance studies that are already planned as was also done in the prevalence study in layers. However, there is no urgent need regarding the fact that the numbers of ducks and turkeys are relatively small in the Netherlands and the number of human psittacosis cases are low and without a clear association with poultry (39).

From the perspective of general preparedness, it would be helpful if serological tools would become available that at least can discriminate between Chlamydia gallinacea and Chlamydia psittaci in poultry. This topic was not further discussed in this thesis, but discriminatory diagnostic detection of different Chlamydia species relies on molecular tests, which only give information about the current status of an animal. To identify any previous exposure to Chlamydia, the availability of a discriminatory serological test would be of added value for any future surveillance studies. Some promising results in chickens have already been obtained with a multiplex serological assay that uses synthetic peptides developed for mammalian serology (43), but this assay needs further validation.

At last, it would be interesting to further investigate the host-pathogen interactions of $C$. gallinacea in the gut and to study co-infections with other poultry pathogens. This will not be easy and will require a more fundamental approach, but it is intriguing how C. gallinacea can survive in gut epithelial cells without causing any visible signs of inflammation. Most exciting is to speculate if $C$. gallinacea infections could have a beneficial effect for the host. For example, do C. gallinacea infections play a role in biological processes like trained immunity? This is the concept that certain stimuli can shape or adapt the innate immune system which results in better responsiveness and has been shown in exposure to bacillus Calmette-Guerin (BCG) vaccine (44). Trying to answer more fundamental questions about the interaction of $C$. gallinacea with its host might help us to better understand the grey area between health and disease especially in the gut with its huge microbiome. 


\section{Take home messages}

- Chlamydia infections in Dutch chickens should not be considered a current One Health threat as Chlamydia psittaci could not be detected in a prevalence study, nor is there evidence that the highly prevalent Chlamydia gallinacea causes acute disease in chickens or has a zoonotic potential.

- Future introduction of C. psittaci at chickens farms via contact with wild birds cannot be ruled out and requires transmission studies to further investigate the susceptibility of chickens to $C$. psittaci infection and subsequent infection dynamics.

- It is intriguing how C. gallinacea is able to survive in gut epithelial cells without causing any visible signs of inflammation or acute disease. This requires more fundamental research into host-pathogen interactions of $C$. gallinacea, which may help to better understand the grey area between health and disease.

- The prevalence of Chlamydia in duck and turkey farms was not investigated in this thesis, but there is no urgent need regarding the fact that the number of duck and turkey farms are relatively small in the Netherlands. 


\section{References}

1. Laroucau K, Vorimore F, Aaziz R, Berndt A, Schubert E, Sachse K. Isolation of a new chlamydial agent from infected domestic poultry coincided with cases of atypical pneumonia among slaughterhouse workers in France. Infection, genetics and evolution : journal of molecular epidemiology and evolutionary genetics in infectious diseases. 2009;9(6):1240-7.

2. Sachse K, Laroucau K, Vanrompay D. Avian Chlamydiosis. Current Clinical Microbiology Reports. 2015;2(1):10-21.

3. Lagae S, Kalmar I, Laroucau K, Vorimore F, Vanrompay D. Emerging Chlamydia psittaci infections in chickens and examination of transmission to humans. Journal of medical microbiology. 2014;63(Pt 3):399-407.

4. Ornelas-Eusebio E, Garcia-Espinosa G, Vorimore F, Aaziz R, Durand B, Laroucau K, et al. Crosssectional study on Chlamydiaceae prevalence and associated risk factors on commercial and backyard poultry farms in Mexico. Preventive veterinary medicine. 2020;176:104922.

5. Guo W, Li J, Kaltenboeck B, Gong J, Fan W, Wang C. Chlamydia gallinacea, not C. psittaci, is the endemic chlamydial species in chicken (Gallus gallus). Scientific reports. 2016;6:19638.

6. Hulin V, Oger S, Vorimore F, Aaziz R, de Barbeyrac B, Berruchon J, et al. Host preference and zoonotic potential of Chlamydia psittaci and C. gallinacea in poultry. Pathogens and disease. 2015;73(1):1-11.

7. Harkinezhad T, Geens T, Vanrompay D. Chlamydophila psittaci infections in birds: a review with emphasis on zoonotic consequences. Veterinary microbiology. 2009;135(1-2):68-77.

8. Page LA. Experimental Ornithosis in Turkeys. Avian diseases. 1959;3(1):51-66.

9. Vanrompay D, Ducatelle R, Haesebrouck F, Hendrickx W. Primary pathogenicity of an European isolate of Chlamydia psittaci from turkey poults. Veterinary microbiology. 1993;38(1-2):103-13.

10. Thierry S, Vorimore F, Rossignol C, Scharf S, Sachse K, Berthon P, et al. Oral Uptake of Chlamydia psittaci by Ducklings Results in Systemic Dissemination. PloS one. 2016;11(5):e0154860.

11. Salisch H VMK, Ryll M, Hinz K-H. Chlamydial infections of poultry and human health. World's Poultry Science Journal. 1996;52(3):279-308.

12. Szymanska-Czerwinska M, Mitura A, Zareba K, Schnee C, Koncicki A, Niemczuk K. Poultry in Poland as Chlamydiaceae Carrier. J Vet Res. 2017;61(4):411-9.

13. Reinhold P, Sachse K, Kaltenboeck B. Chlamydiaceae in cattle: commensals, trigger organisms, or pathogens? Veterinary journal. 2011;189(3):257-67.

14. Rank RG, Yeruva L. Hidden in plain sight: chlamydial gastrointestinal infection and its relevance to persistence in human genital infection. Infection and immunity. 2014;82(4):1362-71.

15. Panzetta ME, Valdivia RH, Saka HA. Chlamydia Persistence: A Survival Strategy to Evade Antimicrobial Effects in-vitro and in-vivo. Frontiers in microbiology. 2018;9:3101.

16. Bavoil PM. What's in a word: the use, misuse, and abuse of the word "persistence" in Chlamydia biology. Front Cell Infect Microbiol. 2014;4:27.

17. Yeruva L, Spencer N, Bowlin AK, Wang Y, Rank RG. Chlamydial infection of the gastrointestinal tract: a reservoir for persistent infection. Pathogens and disease. 2013;68(3):88-95. 
18. You J, Wu Y, Zhang X, Wang X, Gong J, Zhao Z, et al. Efficient fecal-oral and possible vertical, but not respiratory, transmission of emerging Chlamydia gallinacea in broilers. Veterinary microbiology. 2019;230:90-4.

19. Kogut $\mathrm{MH}$, Genovese $\mathrm{KJ}$, Swaggerty $\mathrm{CL}$, He H, Broom L. Inflammatory phenotypes in the intestine of poultry: not all inflammation is created equal. Poultry science. 2018;97(7):2339-46.

20. Smit LA, van der Sman-de Beer F, Opstal-van Winden AW, Hooiveld M, Beekhuizen J, Wouters IM, et al. $Q$ fever and pneumonia in an area with a high livestock density: a large population-based study. PloS one. 2012;7(6):e38843.

21. Smit LAM, Boender GJ, de Steenhuijsen Piters WAA, Hagenaars TJ, Huijskens EGW, Rossen JWA, et al. Increased risk of pneumonia in residents living near poultry farms: does the upper respiratory tract microbiota play a role? Pneumonia. 2017;9(1):3.

22. Post PM, Hogerwerf L, Huss A, Petie R, Boender GJ, Baliatsas C, et al. Risk of pneumonia among residents living near goat and poultry farms during 2014-2016. PloS one. 2019;14(10):e0223601.

23. Yin L, Lagae S, Kalmar I, Borel N, Pospischil A, Vanrompay D. Pathogenicity of low and highly virulent Chlamydia psittaci isolates for specific-pathogen-free chickens. Avian diseases. 2013;57(2):242-7.

24. Yin L, Kalmar ID, Lagae S, Vandendriessche S, Vanderhaeghen W, Butaye $P$, et al. Emerging Chlamydia psittaci infections in the chicken industry and pathology of Chlamydia psittaci genotype $B$ and $D$ strains in specific pathogen free chickens. Veterinary microbiology. 2013;162(2-4):740-9.

25. Takahashi T, Takashima I, Hashimoto N. Shedding and transmission of Chlamydia psittaci in experimentally infected chickens. Avian diseases. 1988;32(4):650-8.

26. Takahashi T, Takashima I, Hashimoto N. A chicken model of systemic infection with Chlamydia psittaci: comparison of the virulence among avian and mammalian strains. Nihon juigaku zasshi The Japanese journal of veterinary science. 1988;50(3):622-31.

27. Suwa T, Ando S, Hashimoto N, Itakura C. Pathology of experimental chlamydiosis in chicks. Nihon juigaku zasshi The Japanese journal of veterinary science. 1990;52(2):275-83.

28. Kaleta EF, Taday EM. Avian host range of Chlamydophila spp. based on isolation, antigen detection and serology. Avian pathology : journal of the WVPA. 2003;32(5):435-61.

29. Heddema ER, Ter Sluis S, Buys JA, Vandenbroucke-Grauls CM, van Wijnen JH, Visser CE. Prevalence of Chlamydophila psittaci in fecal droppings from feral pigeons in Amsterdam, The Netherlands. Applied and environmental microbiology. 2006;72(6):4423-5.

30. Burt SA, Roring RE, Heijne M. Chlamydia psittaci and C. avium in feral pigeon (Columba livia domestica) droppings in two cities in the Netherlands. The Veterinary quarterly. 2018:1-4.

31. Grimes JE, Owens KJ, Singer JR. Experimental transmission of Chlamydia psittaci to turkeys from wild birds. Avian diseases. 1979;23(4):915-26.

32. Grimes J. Transmission of chlamydiae from grackles to turkeys. Avian diseases. 1978:308-12.

33. Gonzales JL, Stegeman JA, Koch G, de Wit SJ, Elbers ARW. Rate of introduction of a low pathogenic avian influenza virus infection in different poultry production sectors in the Netherlands. Influenza and other respiratory viruses. 2013;7(1):6-10. 
34. Bouwstra R, Gonzales JL, de Wit S, Stahl J, Fouchier RAM, Elbers ARW. Risk for Low Pathogenicity Avian Influenza Virus on Poultry Farms, the Netherlands, 2007-2013. Emerging infectious diseases. 2017;23(9):1510-6.

35. Vanrompay D. Avian Chlamydiosis. Diseases of Poultry2020. p. 1086-107.

36. Hogerwerf L, Roof I, de Jong MJK, Dijkstra F, van der Hoek W. Animal sources for zoonotic transmission of psittacosis: a systematic review. BMC infectious diseases. 2020;20(1):192.

37. Gaede W, Reckling KF, Dresenkamp B, Kenklies S, Schubert E, Noack U, et al. Chlamydophila psittaci infections in humans during an outbreak of psittacosis from poultry in Germany. Zoonoses Public Health. 2008;55(4):184-8.

38. Heddema ER, van Hannen EJ, Bongaerts M, Dijkstra F, Ten Hove RJ, de Wever B, et al. Typing of Chlamydia psittaci to monitor epidemiology of psittacosis and aid disease control in the Netherlands, 2008 to 2013 . Euro surveillance : bulletin Europeen sur les maladies transmissibles = European communicable disease bulletin. 2015;20(5):21026.

39. Vlaanderen F, Cuperus T, Keur I, de Rosa M, Rozendaal H, Friesema I, et al. Staat van Zoönosen 2019. State of Zoonoses 2019: Rijksinstituut voor Volksgezondheid en Milieu RIVM; 2020.

40. Vanrompay D, Mast J, Ducatelle R, Haesebrouck F, Goddeeris B. Chlamydia psittaci in turkeys: pathogenesis of infections in avian serovars A, B and D. Veterinary microbiology. 1995;47(3):24556.

41. Loock MV, Geens T, Smit LD, Nauwynck H, Empel PV, Naylor C, et al. Key role of Chlamydophila psittaci on Belgian turkey farms in association with other respiratory pathogens. Veterinary microbiology. 2005;107(1):91-101.

42. Hogerwerf L, Holstege MMC, Beninca E, Dijkstra F, van der Hoek W. Temporal and spatial analysis of psittacosis in association with poultry farming in the Netherlands, 2000-2015. BMC infectious diseases. 2017;17(1):519.

43. Sachse K, Rahman KS, Schnee C, Muller E, Peisker M, Schumacher T, et al. A novel synthetic peptide microarray assay detects Chlamydia species-specific antibodies in animal and human sera. Scientific reports. 2018;8(1):4701.

44. Netea MG, Dominguez-Andres J, Barreiro LB, Chavakis T, Divangahi M, Fuchs E, et al. Defining trained immunity and its role in health and disease. Nat Rev Immunol. 2020;20(6):375-88. 


\section{Summary}

Chlamydia gallinacea and Chlamydia psittaci are intracellular bacteria belonging to the Chlamydiaceae family and are a cause of avian chlamydiosis in poultry. C. psittaci was considered the predominant chlamydial species in poultry until Chlamydia gallinacea was discovered in 2009. C. psittaci occurs worldwide, is zoonotic and has a wide host range. Depending on the $C$. psittaci strain, host, age of the host and environmental factors (stress), infections in poultry can be asymptomatic or result in more severe issues, such as acute respiratory distress and mortality. Infections in humans can result in severe pneumonia. C. gallinacea is widespread in chickens, and infections do not seem to result in disease, but reduced weight gain has been observed in broilers. Studies about the pathogenic potential of $C$. gallinacea are still limited, and any zoonotic potential has yet to be determined.

Prior observations in poultry that contributed to the questions addressed in this thesis were as follows. It was unknown if C. gallinacea and C. psittaci also occur in Dutch poultry, however, since 2010, C. psittaci infections in chickens were reported in surrounding countries. Moreover, in 2012, a Dutch study reported a higher number of pneumonia cases in residents living near poultry farms. At the time, the cause of these pneumonia cases was unknown, but Chlamydia was proposed as potentially playing a role.

The aim of this study was to gain insight into the prevalence of Chlamydia in Dutch chickens and to investigate the pathogenic potential of $C$. gallinacea in chickens. Finally, it was investigated whether a previous C. gallinacea infection in chickens could protect against a C. psittaci infection.

Chapter 2 investigates the prevalence of Chlamydia in Dutch layers. C. gallinacea DNA was detected in pooled faecal samples on 71 of 151 layer farms, but C. psittaci DNA was not detected. No association between clinical signs (i.e. respiratory symptoms, nasal and ocular discharge, mortality) and the presence of $C$. gallinacea was found.

Chapter 3 describes two novel C. gallinacea strains (NL_G47 and NL_F725) that were isolated from the caeca of seemingly healthy chickens. Subsequent genomic analysis showed both strains were unique and possessed the hallmark genetic coding for known and potential virulence factors found in C. psittaci, albeit to a reduced number of orthologs or alleles. Whether these genetic differences contribute to phenotypic differences is unclear. Phenotypic analyses in embryonated specific pathogen free (SPF) eggs revealed C. gallinacea induced mortality, but to a lesser extent than C. psittaci. 
Subsequent experiments with C. gallinacea strain NL_G47 in six-week-old SPF layers, detailed in Chapter 4, confirmed observations from field studies that C. gallinacea infections do not result in acute clinical disease. In this study, layers were orally inoculated, which resulted in throat and cloacal shedding, and infection of epithelial cells of the jejunum, ileum and caecum without signs of clinical disease, nor were there macroscopic or histologic signs of inflammation. On day 11 post inoculation, chlamydial antigen was co-localized within macrophages in the lamina propria and follicular dendritic cells in the caecal tonsil and, from day 7 onwards, a rise in antibody titre was shown.

After the finding in Chapter 2, that C. gallinacea was highly prevalent on Dutch layer farms, we hypothesize in Chapter $\mathbf{5}$ that the absence of $C$. psittaci could be explained by cross protection between C. gallinacea and C. psittaci. Chickens were therefore first inoculated with C. gallinacea NL_G47 and subsequently inoculated with either C. gallinacea NL_ F725 or C. psittaci. The inoculations did not result in a difference in shedding or tissue dissemination pattern of $C$. psittaci between the groups. Thus, the absence of $C$. psittaci in the prevalence study could not be explained by cross protection from previous $C$. gallinacea infections. However, a prior C. gallinacea infection did partially protect against a new $C$. gallinacea infection based on the PCR results of cloacal shedding.

The last chapter (Chapter 6) discusses whether the results in laying hens can be translated to broilers or other poultry species. Furthermore, it addresses whether $C$. gallinacea should be considered a pathogen, and how the absence of $C$. psittaci should be interpreted regarding animal and public health. The current conclusion is that Chlamydia infections in Dutch chickens cannot be considered a One Health problem. Infections with C. gallinacea do not lead to clinical disease in chickens, and C. psittaci could not be detected in a prevalence study in layers. However, these results do not exclude the future introduction of $C$. psittaci in chickens, nor its occurrence in other poultry species. These questions would require further research. It is also intriguing that $C$. gallinacea is able to replicate intracellularly without causing visible signs of inflammation, a phenomenon that is observed in other Chlamydia and intracellular bacterial infections as well. This requires more fundamental research into host-pathogen interactions, which may help to better understand the grey area between health and disease. 


\section{Samenvatting}

Chlamydia gallinacea en Chlamydia psittaci zijn beide intracellulaire bacteriën die tot de familie van de Chlamydiaceae behoren en aviaire chlamydiose in pluimvee kunnen veroorzaken. Tot de ontdekking van C. gallinacea in 2009 werd aangenomen dat C. psittaci de belangrijkste Chlamydia soort in pluimvee was. C. psittaci komt wereldwijd voor, kan van dier op mens worden overgedragen (zoönose) en kent een breed scala aan gastheren. Infecties in pluimvee kunnen zonder verschijnselen verlopen, maar ook tot ernstige verschijnselen als benauwdheid en sterfte leiden. Dit hangt af van de $C$. psittaci stam, gastheer, leeftijd van de gastheer en omgevingsfactoren (stress). Infecties in mensen kunnen resulteren in een ernstige longontsteking. C. gallinacea komt vooral voor in kippen en infecties lijken niet tot ziekte te leiden. Wel is bij vleeskuikens verminderde groei waargenomen. Naar het ziekteverwekkend vermogen van C. gallinacea is echter nog maar beperkt onderzoek gedaan. Ook het zoönotisch potentieel is nog niet opgehelderd.

Voorafgaand aan het onderzoek in dit proefschrift was onbekend in hoeverre $C$. gallinacea en C. psittaci ook in Nederlands pluimvee vóórkomen, terwijl er na 2010 in omliggende landen wel degelijk C. psittaci in kippen werd aangetoond. Bovendien werd in een Nederlandse studie uit 2012 een hoger aantal gevallen van longontsteking bij omwonenden van pluimveebedrijven gerapporteerd. De mogelijke oorzaak van deze gevallen van longontsteking was op dat moment onbekend, maar een hypothese was dat C. psittaci of C. gallinacea wellicht een rol zouden kunnen spelen.

Het doel van dit onderzoek was om het vóórkomen van Chlamydia in kippen in Nederland in kaart te brengen, inzicht te krijgen in het ziekteverwekkend vermogen van C. gallinacea door Nederlandse stammen te kweken, te karakteriseren en te testen in een model met eieren. Daarnaast is experimenteel onderzoek in kippen uitgevoerd. Als laatste is onderzocht of een doorgemaakte $C$. gallinacea infectie in kippen, een infectie met $C$. psittaci kan voorkomen.

In het onderzoek naar het vóórkomen, dat beschreven is in hoofdstuk 2, werd op 71 van de 151 onderzochte leghennenbedrijven met een PCR test. C. gallinacea aangetoond in fecesmonsters. C. psittaci werd in geen van de onderzochte monsters gevonden. De aanwezigheid van C. gallinacea kon niet worden gerelateerd aan het optreden van klinische verschijnselen zoals neus- of ooguitvloeiing, benauwdheid of diarree, noch met verhoogde sterftecijfers.

In hoofdstuk 3 is beschreven hoe uit het caecum van gezonde leghennen, afkomstig van twee verschillende koppels, twee genetisch verschillende isolaten van $C$. gallinacea werden gekweekt. Verder onderzoek aan het genoom liet zien dat C. gallinacea genen 
heeft die in Chlamydia gerelateerd worden aan virulentie. C. gallinacea heeft wel minder van dit soort genen dan C. psittaci, maar de vraag is in hoeverre deze genetische verschillen samenhangen met het ontstaan van ziekte in dieren. In kippeneieren kan experimentele infectie met C. gallinacea via de dooierzak, sterfte van het embryo veroorzaken.

In hoofdstuk 4 is verder onderzoek gedaan naar het ziekteverwekkend vermogen van C. gallinacea in kippen. Na orale toediening, leidde een infectie met C. gallinacea niet tot acute klinische ziekte. C. gallinacea kon vooral worden aangetoond in epitheelcellen van het jejunum, ileum en caecum zonder zichtbare verschijnselen van ontsteking. Daarnaast werd C. gallinacea in macrofagen en dendritische cellen van de caecale tonsil aangetoond en werd een toename van antilichamen gemeten. Verspreiding van C. gallinacea naar andere organen werd niet aangetoond.

Als laatste is in hoofdstuk 5 onderzocht of een doorgemaakte C. gallinacea infectie de kans op een infectie met $C$. psittaci kon verlagen. De resultaten van de infectie experimenten lieten geen onderbouwing zien voor deze veronderstelling. De afwezigheid van C. psittaci in de prevalentiestudie uit hoofdstuk 2, kan dus niet worden verklaard door mogelijke kruisbescherming (door de hoge prevalentie van C. gallinacea). Na een doorgemaakte $C$. gallinacea infectie is de uitscheiding via de cloaca bij een nieuwe C. gallinacea infectie met een andere stam wel lager.

In de algemene discussie (hoofdstuk 6) wordt besproken in hoeverre de resultaten over het voorkomen van Chlamydia in leghennen ook vertaald kunnen worden naar vleeskuikens of andere pluimveesoorten. Daarnaast wordt ingegaan op de vraag of C. gallinacea nu een ziekteverwekker is of niet en wat het niet aantonen van C. psittaci betekent voor zowel de dier- als volksgezondheid. De eindconclusie is dat Chlamydia infecties in kippen in Nederland op dit moment geen One Health probleem vormen. De huidige resultaten sluiten toekomstige introductie van de zoönotisch C. psittaci in kippen echter niet uit. Daarvoor zou verder onderzoek nodig zijn. Ook is het interessant dat $C$. gallinacea in staat is om zich intracellulair te vermeerderen zonder dat dit tot zichtbare ziekte of ontsteking leidt. Dit geldt overigens voor meer Chlamydia infecties en vraagt om fundamenteel onderzoek naar de gastheer-pathogeen interacties om beter te begrijpen wat ziek en gezond is. 


\section{Dankwoord}

Het schrijven van een proefschrift lijkt misschien een soloproject, maar dat is het zeker niet. Met dit dankwoord wil ik iedereen bedanken die direct of indirect betrokken is geweest, ook als ik je naam hier niet noem.

Voor mij startte dit avontuur in 2012 toen ik als veterinair microbioloog in opleiding (VMIO) aan de slag ging bij de afdeling bacteriologie en TSE's van het Centraal Veterinair Instituut (nu Wageningen Bioveterinary Research) aan de Edelhertweg in Lelystad. Hendrik-Jan motiveerde mij om het VMIO-traject uit te bouwen tot een promotietraject. Hendrik-Jan dank daarvoor, je was een beetje mijn schaduw-copromotor.

Mirjam, Ad en Jeanet, hebben mij vervolgens als promotor en copromotoren naar de eindstreep begeleid. Jeanet, ik kon gedurende het hele traject altijd bij je aankloppen met alle soorten vragen. Je hebt me vanaf het begin af aan gesteund. Ad, jij raakte iets later betrokken. Ik heb vooral van je geleerd om op je eigen resultaten te durven vertrouwen en een open blik te houden. Mirjam, jij sloot als promotor als laatste aan in dit traject. Je hebt me met jouw nuchterheid en directe aanpak enorm geholpen om dit boekje ook echt af te maken. Dank voor jullie begeleiding.

Ook bij het verzamelen van alle data en de analyse daarvan, heb ik gedurende het traject veel hulp gehad. Mede dankzij Joke, Kitty, Annika, Marieke en Ben kon ik monsters van een NVWA-RIVM studie gebruiken voor de prevalentiestudie uit hoofdstuk 2 van dit boekje. Helmi heeft ervoor gezorgd dat al deze monsters zijn getest op Chlamydia en Eric heeft me geholpen met het kaartje van Nederland. Dank daarvoor.

Met dank aan Francisca van de Faculteit Diergeneeskunde, kon ik monsters van kippen verzamelen voor de studie uit hoofdstuk 3. Dit was ook niet mogelijk geweest zonder de hulp van de dierverzorgers bij Landbouwhuisdieren (o.a. Freek, Marc en Carmen). Zelfs rondom de kerstdagen stonden zij klaar om te helpen met het swabben van kippen. Diana heeft me geholpen met het opzetten van de eikweek en via Agnes van WLR kon ik mobiele ei-incubators lenen. Dank daarvoor. Dank ook aan de collega 's die in het weekend eitjes hebben geschouwd.

En natuurlijkdankaan Famkeen Annemieke die me geholpen hebben met de experimenten in eieren en de isolatie van 2 Nederlandse C. gallinacea stammen. Annemieke, jij hebt me daarnaast de beginselen van de celkweek bijgebracht en ingewerkt op het BSL-3 lab. Voor de celkweek van Chlamydia zijn we samen naar het FLI in Jena en de Universiteit van Gent geweest. Een mooi avontuur. Ik ben heel blij dat je me nu samen met Lars, in de rol van paranimf, helpt met de laatste loodjes. 
From FLI, I would like to thank Konrad Sachse and Christiane Schnee not only for their hospitality, but also for providing us several Chlamydia reference strains. Van de Universiteit Gent wil ik Daisy Vanrompay en Annelien Dumont bedanken voor de gastvrije ontvangst en advies op het gebied van de celkweek.

Frank, Arie, Quillan en Mike wil ik bedanken voor het NGS werk (zowel Illumina als Nanopore, voor de echte kenners). Alexander, Martina and Yvonne, without you these data wouldn't have been analyzed. Thank you for your help, but also for your enthusiasm.

De studies in hoofdstuk 4 en 5 waren er niet geweest zonder de inzet van de collega 's van zowel de stallen op de Runderweg als de Houtribweg. Vooral de experimenten op BSL-3 niveau vragen veel expertise, maar zijn ook fysiek belastend vanwege het dragen van beschermende kleding (en meerdere keren douchen op een dag). Dank voor jullie inzet. Lucien, Rob, Sebastiaan, Corrie en Lars bedankt voor jullie hulp bij de secties van de dieren en het maken van alle coupes. Lucien, ik ben trots op de mooie foto 's die dat heeft opgeleverd.

Dank aan alle collega 's van DSU en DCO die betrokken zijn geweest bij het opwerken en testen van monsters. Vooral Esther, Eugenie, Herma, Irene, Marianne en Robin hebben heel veel swabs voorbij zien komen. Herma jij dacht vaak actief mee over de proefopzet, vanwege eerdere ervaring met werk aan Chlamydia. Dank daarvoor.

Voor het testen van sera kon ik gebruik van een ELISA die voortkwam uit de projecten SeroChlam en ChlamSero. Fimme-Jan en Ruth, dank voor het opzetten hiervan. En natuurlijk ook de andere collega 's in deze projecten voor de samenwerking. Hopelijk kunnen we nog voor het einde van dit jaar een manuscript indienen met de resultaten die het werk aan Chlamydia serologie heeft opgeleverd.

Daarnaast zijn er de nodige collega 's die hebben geholpen door bijvoorbeeld het bestellen van materialen (Conny, Lars, René), het boeken van reizen voor congressen (alle behulpzame collega 's van het secretariaat), het verbeteren van figuren (Monique van communicatie) en het proeflezen van manuscripten (Mariëlle, Fimme-Jan). Ook jullie wil ik bedanken. Willie en later Matthijn dank voor de steun vanuit de WOT. En niet te vergeten dank aan Hetty, die mij de laatste 2 jaar als afdelingshoofd de ruimte heeft gegeven om mijn proefschrift af te kunnen maken.

Een boekje komt niet tot stand zonder financiering. Vooral het project 'Chlamypluim', gefinancierd door het ministerie van LNV, was belangrijk voor het werk in dit proefschrift. Daarnaast was het opgebouwde netwerk uit het door ZonMW gefinancierde project 'Plat4m-2bt-Psittacosis' van belang. Dit project startte in 2014 en bestond uit een breed consortium met o.a. WBVR, RIVM, GGD, NVWA, GD, Amsterdam UMC en verschillende 
medisch microbiologische labs als partners. Ik wil alle deelnemers aan dit netwerk hartelijk bedanken, want deze samenwerking heeft zonder meer aan de basis gestaan van de ideeën en het onderzoek in dit proefschrift.

Ook de carpool wil ik bedanken. Vanaf mijn eerste dag in Lelystad kon ik dankzij Roselinde aansluiten bij de carpool vanuit Utrecht. Dat betekende elke ochtend om 7.15 uur vertrek vanaf winkelcentrum "De Gaard". Niet echt een ideaal tijdstip voor iemand die zichzelf niet als ochtendmens zou omschrijven. De koffie maakte een hoop goed en bij deze dus nog excuses voor de keren dat ik ietwat aan de late kant was. Roselinde, Jan Rinze, Esther, Saskia, Judith, Marjolijn, Catherine, Jesus en Lars, dank voor de vele gesprekken en natuurlijk ook de etentjes, pasta party en BBQ.

En dan mijn kamergenoten in de loop der jaren: Miriam, Marc, Aart, Rianka, Marjolijn, Alexander en sinds kort Marleen. Dank dat jullie met me hebben meegedacht of me soms de nodige peptalk hebben gegeven. Miriam, jij bent vanaf het begin af aan betrokken, ook als begeleider in het VMIO-traject. Dank daarvoor.

Dank ook aan de collega's die onderdeel uit maken van de intervisie groep, de andere PhD's binnen WBVR en de groep met jonge projectleiders en postdocs (alhoewel activiteiten sinds COVID min of meer stil liggen). En natuurlijk dank aan alle andere fijne en betrokken collega 's die ervoor gezorgd hebben dat ik mij bijzonder thuis voel bij WBVR. Door het VMIO-traject heb ik op bijna alle afdelingen wel eens een dag meegelopen en hebben jullie mij wegwijs gemaakt in de wereld van de microbiologie.

Dan mijn vrienden van de studie diergeneeskunde in Gent of Utrecht, mijn studentenhuis op het IBB, een reis naar Cuba of etentjes met 'wat schaft de pot': jullie hebben mij door dit promotietraject en de COVID maatregelen de afgelopen 1,5 jaar misschien iets minder vaak gezien, maar zijn altijd betrokken geweest. lk hoop dat we in 2022 weer de nodige etentjes kunnen inhalen of het jaarlijkse weekendje weg.

Mijn ouders, broer en schoonouders, die ik een aantal jaar geleden tijdens de familiedag van WBVR heb kunnen laten zien wat mijn werk inhoudt. Zonder de steun van mijn ouders, had ik dit nooit kunnen bereiken.

En als laatste Catharien, je was de afgelopen 1,5 jaar niet alleen mijn partner maar ook mijn belangrijkste collega. Je kent me niet anders dan dat ik aan het promoveren ben. Je denkt met me mee en samen zorgen we voor voldoende ontspanning door wandelingen met de hond, fietstochten, kanotochten en hopelijk binnenkort een nieuw buitenland avontuur met een zeilreis in Noorwegen. Ik hoop ook dat we over een paar jaar een vergelijkbaar feestje kunnen vieren, als jij je master Rechten haalt. 


\section{About the author}

Marloes Heijne (1982) started her degree in Veterinary Medicine at the Ghent University in Belgium. After finishing her first year in Ghent, she returned to the Netherlands to study Veterinary Medicine at Utrecht University. Besides her study in Veterinary Medicine, Marloes finished a minor in public administration and organisation science at the Utrecht University School of Governance. In 2005, she completed her Master's thesis, on avian influenza dynamics in poultry and waterfowl with a focus on China, as part of a three-month internship at the Food and Agricultural Organisation (FAO) in Rome. In 2008, Marloes graduated as a veterinarian specialising in Farm Animal Health.

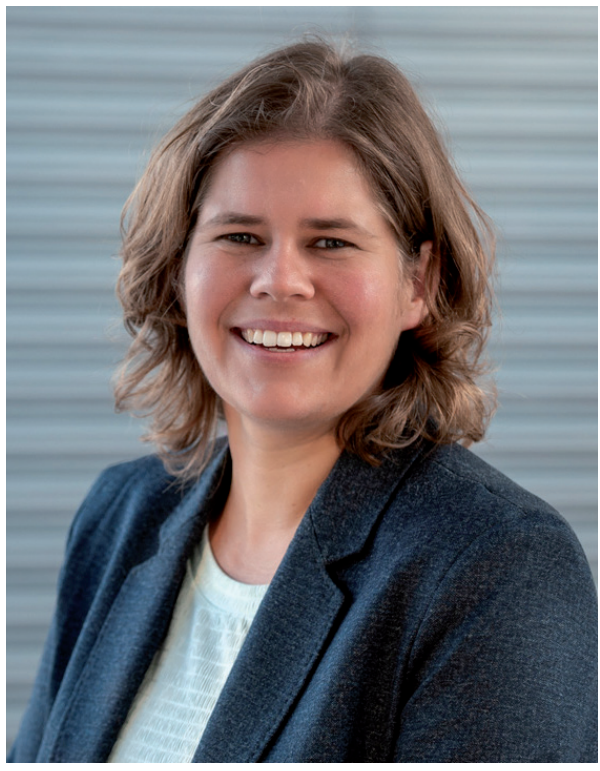

From 2008 to 2011 she worked as a farm animal veterinarian at the 'Dierenartspraktijk van Waard tot Klif' in Friesland. In 2011, she returned to Utrecht to work as a lecturer at the Farm Animal Health department of the Faculty of Veterinary Medicine. In her current job at Wageningen Bioveterinary Research (WBVR) in Lelystad, she is about to finish her training as a veterinary microbiologist. The research part of this training has continued into her PhD. Marloes participated in several research projects, including a ZonMW funded research project 'Plat4m-2bt-psittacosis'. She also acquired funding from the Dutch Ministry of Agriculture for the work presented in Chapters 3, 4 and 5 of this thesis. She has been a project leader on statutory tasks for notifiable bacterial animal diseases and zoonoses including avian chlamydiosis at WBVR since 2019. 


\section{List of publications}

Ciuria S, Brouwer MSM, de Gier MM, van Zeeland Y, Bossers A, Prähauser B, Schädler J, Hatt J-M, Heijne M, Borel N. Chlamydia caviae in Swiss and Dutch Guinea PigsOccurrence and Genetic Diversity. Pathogens. 2021; 10(10):1230. https://doi.org/10.3390/ pathogens10101230

Heijne M, Jelocnik M, Umanets A, Brouwer MSM, Dinkla A, Harders F, van Keulen LJM, Roest HJ, Schaafsma F, Velkers FC, van der Goot JA, Pannekoek Y, Koets AP. Genetic and phenotypic analysis of the pathogenic potential of two novel Chlamydia gallinacea strains compared to Chlamydia psittaci. Sci Rep. 2021 Aug 13;11(1):16516. doi: 10.1038/s41598021-95966-9. PMID: 34389764; PMCID: PMC8363750.

Heijne M, van der Goot J, Buys H, Dinkla A, Roest HJ, van Keulen L, Koets A. Pathogenicity of Chlamydiagallinacea in chickens after oral inoculation. Vet Microbiol. 2021 Aug;259:109166. doi: 10.1016/j.vetmic.2021.109166. Epub 2021 Jun 25. PMID: 34217040.

Kik M, Heijne M, IJzer J, Grinwis G, Pannekoek Y, Gröne A. Fatal Chlamydia avium Infection in Captive Picazuro Pigeons, the Netherlands. Emerg Infect Dis. 2020 Oct;26(10):2520-2522. doi: 10.3201/eid2610.191412. PMID: 32946739; PMCID: PMC7510720.

Polkinghorne A, Borel N, Heijne M, Pannekoek Y. New evidence for domesticated animals as reservoirs of Chlamydia-associated community-acquired pneumonia. Clin Microbiol Infect. 2019 Feb;25(2):131-132. doi: 10.1016/j.cmi.2018.10.015. Epub 2018 Oct 28. PMID: 30394363.

Burt SA, Röring RE, Heijne M. Chlamydia psittaci and C. avium in feral pigeon (Columba livia domestica) droppings in two cities in the Netherlands. Vet Q. 2018 Dec;38(1):6366. doi: 10.1080/01652176.2018.1482028. Epub 2018 Jun 5. PMID: 29806552; PMCID: PMC6831002.

Heijne M, van der Goot JA, Fijten H, van der Giessen JW, Kuijt E, Maassen CBM, van Roon A, Wit B, Koets AP, Roest HIJ. A cross sectional study on Dutch layer farms to investigate the prevalence and potential risk factors for different Chlamydia species. PLoS One. 2018 Jan 11;13(1):e0190774. doi: 10.1371/journal.pone.0190774. PMID: 29324884; PMCID: PMC5764275.

Ramakers BP, Heijne $\mathbf{M}$, Lie N, Le TN, van Vliet M, Claessen VPJ, Tolsma PJP, De Rosa M, Roest HIJ, Vanrompay D, Heddema ER, Schneeberger P, Hermans MHA. Zoonotic 
Chlamydia caviae Presenting as Community-Acquired Pneumonia. N Engl J Med. 2017 Sep 7;377(10):992-994. doi: 10.1056/NEJMc1702983. PMID: 28877022.

Heijne M, Hogerwerf L, Dijkstra F, van der Goot JA, Heddema E, Kroneman A, Notermans D, Pannekoek Y, de Rosa M, te Wierik, M, van der Giessen J, Roest H I J \& van der Hoek W. One health-samenwerking in de aanpak van psittacose. Nederlands Tijdschrift voor Medische Microbiologie. 2017 Jun; 25(2);43-48

De Boeck C, Dehollogne C, Dumont A, Spierenburg M, Heijne M, Gyssens I, VAN DER Hilst J, Vanrompay D. Managing a cluster outbreak of psittacosis in Belgium linked to a pet shop visit in The Netherlands. Epidemiol Infect. 2016 Jun;144(8):1710-6. doi: 10.1017/ S0950268815003106. Epub 2015 Dec 16. PMID: 26669637. 





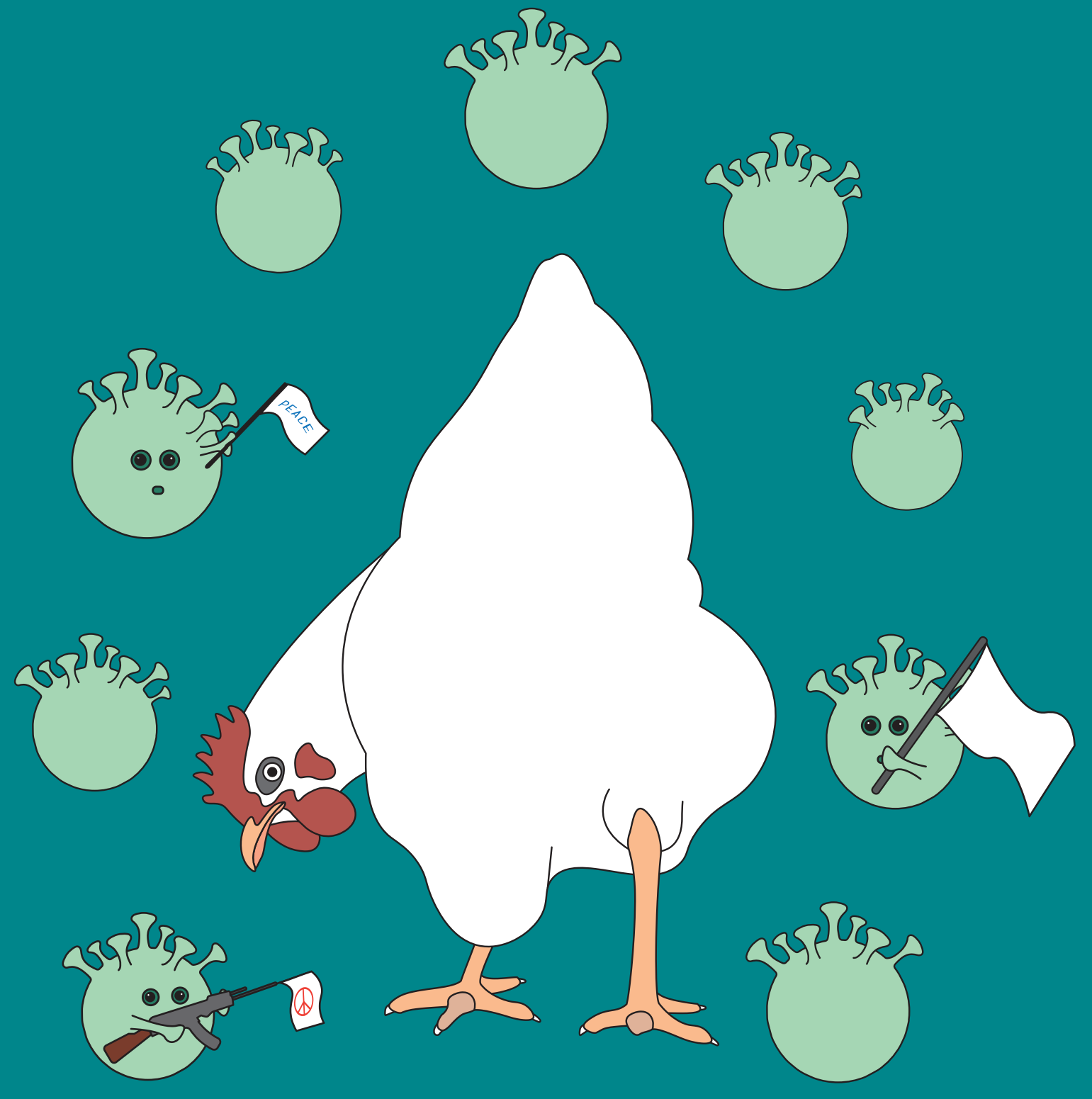

